\title{
Catalytic Asymmetric Synthesis of Protected
}

\section{Tryptophan Regioisomers}

Paul R. Carlier, ${ }^{*,+,+}$ Polo C.-H. Lam, ${ }^{,+*}$ and Dawn M. Wong ${ }^{*}$

${ }^{\dagger}$ Department of Chemistry, Virginia Tech, Blacksburg, Virginia 24061, and ${ }^{\ddagger}$ Department of Chemistry, Hong Kong University of Science and Technology, Clear Water Bay, Kowloon, Hong Kong.

*To whom correspondence should be addressed: pcarlier@vt.edu

\section{Supporting Information}

\section{Table of Contents}

\begin{tabular}{llc}
\hline Description & & Page \\
\hline & Analytical Data for Compounds 4-13, and 22. & S2 \\
Table S1 & $\begin{array}{l}\text { Summary of Experimental Details for HPLC Enantiomeric } \\
\text { Excess Determination. }\end{array}$ & S11 \\
Table S2 & List of Ligands and Complexes in Calculations. & S12 \\
Table S3 & Energies for Compounds 14-21. & S12 \\
& PDB Files for Compounds 14-21. & S13 \\
& ${ }^{1}$ H \& ${ }^{13}$ C NMR Spectra for New Compounds Characterized by & S38 \\
& HRMS
\end{tabular}




\section{Analytical Data for compounds 4-13, and 22.}

General procedure for synthesizing compound 4-13 are found in the main text.

$N$-Boc-2-formylindole (4a). ${ }^{1} \quad$ 2-formylindole $\mathbf{3 a}(98 \mathrm{mg}, 0.68 \mathrm{mmol})$ reacted to give $N$-Boc-2-formylindole $4 a(73 \mathrm{mg}, 44 \%)$ as a yellow solid. $\mathrm{mp}: 70.4-72.6{ }^{\circ} \mathrm{C} ;{ }^{1} \mathrm{H}$ NMR $\left(\mathrm{CDCl}_{3}\right) \delta 1.71\left(\mathrm{~s}, 9 \mathrm{H}, \mathrm{CH}_{3}\right), 7.30\left(\mathrm{t},{ }^{3} J_{\mathrm{HH}}=8.0 \mathrm{~Hz}, 1 \mathrm{H}, \mathrm{Ar}\right), 7.44(\mathrm{~s}, 1 \mathrm{H}, \mathrm{Ar}), 7.48\left(\mathrm{t},{ }^{3} J_{\mathrm{HH}}\right.$ $=8.4 \mathrm{~Hz}, 1 \mathrm{H}, \mathrm{Ar}), 7.68\left(\mathrm{~d},{ }^{3} J_{\mathrm{HH}}=8.1 \mathrm{~Hz}, 1 \mathrm{H}, \mathrm{Ar}\right), 8.17\left(\mathrm{~d},{ }^{3} J_{\mathrm{HH}}=8.5 \mathrm{~Hz}, 1 \mathrm{H}, \mathrm{Ar}\right), 10.44$ (s, $1 \mathrm{H}, \mathrm{CHO}) ;{ }^{13} \mathrm{C} \mathrm{NMR}\left(\mathrm{CDCl}_{3}\right) \delta 28.22,85.56,115.99,116.36,123.11,123.73,127.44$, 128.11, 137.74, 137.77, 149.72, 184.01; MS (CI) $m / z 246[\mathrm{M}+\mathrm{H}]^{+}$.

$N$-Boc-4-formylindole (4b). 4-formylindole $\mathbf{3 b}$ (1.00 g, $6.9 \mathrm{mmol})$ reacted to give $N$ Boc-4-formylindole $4 \mathrm{~b}(1.54 \mathrm{~g}, 91 \%)$ as a yellow oil. ${ }^{1} \mathrm{H} \mathrm{NMR}\left(\mathrm{CDCl}_{3}\right) \delta 1.69(\mathrm{~s}, 9 \mathrm{H}$, $\left.\mathrm{CH}_{3}\right), 7.38\left(\mathrm{dd},{ }^{3} J_{\mathrm{HH}}=3.8 \mathrm{~Hz},{ }^{5} J_{\mathrm{HH}}=0.6 \mathrm{~Hz}, 1 \mathrm{H}, \mathrm{Ar}\right), 7.47\left(\mathrm{dd},{ }^{3} J_{\mathrm{HH}}=8.1 \mathrm{~Hz},{ }^{3} J_{\mathrm{HH}}=7.6\right.$ $\mathrm{Hz}, 1 \mathrm{H}, \mathrm{Ar}), 7.73\left(\mathrm{dd},{ }^{3} J_{\mathrm{HH}}=7.4 \mathrm{~Hz},{ }^{4} J_{\mathrm{HH}}=0.9 \mathrm{~Hz}, 1 \mathrm{H}, \mathrm{Ar}\right), 7.77\left(\mathrm{~d},{ }^{3} J_{\mathrm{HH}}=3.7 \mathrm{~Hz}, 1 \mathrm{H}\right.$, Ar), $8.47\left(\mathrm{br} \mathrm{d},{ }^{3} J_{\mathrm{HH}}=8.3 \mathrm{~Hz}, 1 \mathrm{H}, \mathrm{Ar}\right), 10.24(\mathrm{~s}, 1 \mathrm{H}, \mathrm{CHO}) ;{ }^{13} \mathrm{C} \mathrm{NMR}\left(\mathrm{CDCl}_{3}\right) \delta 28.16$, $84.41,106.57,120.93,123.73,128.25,128.79,128.92,135.79,149.21,192.45 .(11 \mathrm{C}$ found); MS (CI) $m / z 246[\mathrm{M}+\mathrm{H}]^{+}$; HRMS (FAB) calcd for $\mathrm{C}_{14} \mathrm{H}_{16} \mathrm{NO}_{3}[\mathrm{M}+\mathrm{H}]^{+}$ 246.1130, found 246.1123.

N-Boc-6-formylindole (4d). ${ }^{2}$ 6-formylindole $3 \mathbf{d}$ (1.9 g, $\left.13 \mathrm{mmol}\right)$ reacted in the same manner to give $N$-Boc-6-formylindole $4 \mathbf{d}(3.3 \mathrm{~g}, 100 \%)$ as a yellow oil. ${ }^{1} \mathrm{H}$ NMR $\left(\mathrm{CDCl}_{3}\right) \delta 1.71\left(\mathrm{~s}, 9 \mathrm{H}, \mathrm{CH}_{3}\right), 6.65\left(\mathrm{dd},{ }^{3} J_{\mathrm{HH}}=3.8 \mathrm{~Hz},{ }^{5} J_{\mathrm{HH}}=0.6 \mathrm{~Hz}, 1 \mathrm{H}, \mathrm{Ar}\right), 7.67\left(\mathrm{~d},{ }^{3} J_{\mathrm{HH}}\right.$ $=8.2 \mathrm{~Hz}, 1 \mathrm{H}, \mathrm{Ar}), 7.78-7.81(\mathrm{~m}, 2 \mathrm{H}, \mathrm{Ar}), 8.68(\mathrm{~s}, 1 \mathrm{H}, \mathrm{Ar}), 10.08(\mathrm{~s}, 1 \mathrm{H}, \mathrm{CHO}) ;{ }^{13} \mathrm{C}$

${ }^{1}$ Chinchilla, R.; Falvello, L. R.; Galindo, N.; Nájera, C. J. Org. Chem. 2000, 65, 30343041.

${ }^{2}$ Woolridge, E. M.; Rokita, S. E. Tetrahedron Lett. 1989, 30, 6117-6120. 
NMR $\left(\mathrm{CDCl}_{3}\right) \delta 28.16,84.63,107.28,118.67,121.31,122.79,129.56,132.86,134.78$, 135.42, 149.08, 192.14; MS (CI) $m / z, 246[\mathrm{M}+\mathrm{H}]^{+}$.

(Z)- $N^{\alpha}$-acetyl-2,3-dehydro-3-(indol-2'-yl)-alanine, methyl ester (5a). Compound 5a was isolated in $96 \%$ yield by recrystallization from ethyl acetate as a yellow solid. mp: 183.9-184.5 ${ }^{\circ} \mathrm{C} ;{ }^{1} \mathrm{H}$ NMR $\left(\mathrm{CD}_{3} \mathrm{OD}\right) \delta 2.21\left(\mathrm{~s}, 3 \mathrm{H}, \mathrm{CH}_{3}\right), 3.80\left(\mathrm{~s}, 3 \mathrm{H}, \mathrm{OCH}_{3}\right), 6.89(\mathrm{~s}, 1 \mathrm{H}$, Ar), $7.02\left(\mathrm{td},{ }^{3} J_{\mathrm{HH}}=7.5 \mathrm{~Hz},{ }^{4} J_{\mathrm{HH}}=0.7 \mathrm{~Hz}, 1 \mathrm{H}, \mathrm{Ar}\right), 7.17\left(\mathrm{td},{ }^{3} J_{\mathrm{HH}}=7.6 \mathrm{~Hz},{ }^{4} J_{\mathrm{HH}}=1.0 \mathrm{~Hz}\right.$, $1 \mathrm{H}, \mathrm{Ar}), 7.39\left(\mathrm{dd},{ }^{3} J_{\mathrm{HH}}=8.2 \mathrm{~Hz},{ }^{4} J_{\mathrm{HH}}=0.75 \mathrm{~Hz}, 1 \mathrm{H}, \mathrm{Ar}\right), 7.50(\mathrm{~s}, 1 \mathrm{H}, \mathrm{CH}), 7.56(\mathrm{br} \mathrm{d}$, $\left.{ }^{3} J_{\mathrm{HH}}=8.0 \mathrm{~Hz}, 1 \mathrm{H}, \mathrm{Ar}\right) ;{ }^{13} \mathrm{C} \mathrm{NMR}\left(\mathrm{CD}_{3} \mathrm{OD}\right) \delta 22.81,52.89,108.74,112.54,121.15$, $122.15,124.16,124.95,126.93,129.72,132.48,139.10,167.03,173.75 ; \mathrm{MS}$ (CI) $\mathrm{m} / \mathrm{z}$ $259[\mathrm{M}+\mathrm{H}]^{+}$; Anal. Calcd for $\mathrm{C}_{14} \mathrm{H}_{14} \mathrm{~N}_{2} \mathrm{O}_{3}: \mathrm{C}, 65.11 ; \mathrm{H}, 5.46 ; \mathrm{N}, 10.85$. Found: C, $64.85 ; \mathrm{H}, 5.45 ; \mathrm{N}, 10.87$.

(Z)- $N^{\alpha}$-acetyl-2,3-dehydro-3-(indol-4'-yl)-alanine, methyl ester (5b). Compound 5b was isolated in $58 \%$ yield by recrystallization from $\mathrm{MeOH}$ and flash chromatography (diethyl ether) of the remaining mother liquor as a white solid. mp: $183.1-183.5{ }^{\circ} \mathrm{C} ;{ }^{1} \mathrm{H}$ $\operatorname{NMR}\left(\mathrm{CD}_{3} \mathrm{OD}\right) \delta 2.05\left(\mathrm{~s}, 3 \mathrm{H}, \mathrm{CH}_{3}\right), 3.83\left(\mathrm{~s}, 3 \mathrm{H}, \mathrm{OCH}_{3}\right), 6.52\left(\mathrm{dd},{ }^{3} J_{\mathrm{HH}}=3.1 \mathrm{~Hz},{ }^{5} J_{\mathrm{HH}}=\right.$ $0.7 \mathrm{~Hz}, 1 \mathrm{H}, \mathrm{Ar}), 7.14\left(\mathrm{t},{ }^{3} J_{\mathrm{HH}}=7.8 \mathrm{~Hz}, 1 \mathrm{H}, \mathrm{Ar}\right), 7.32\left(\mathrm{~d},{ }^{3} J_{\mathrm{HH}}=3.2 \mathrm{~Hz}, 1 \mathrm{H}, \mathrm{Ar}\right), 7.37(\mathrm{~d}$, $\left.{ }^{3} J_{\mathrm{HH}}=7.5 \mathrm{~Hz}, 1 \mathrm{H}, \mathrm{Ar}\right), 7.42\left(\mathrm{~d},{ }^{3} J_{\mathrm{HH}}=8.1 \mathrm{~Hz}, 1 \mathrm{H}, \mathrm{Ar}\right), 7.79(\mathrm{~s}, 1 \mathrm{H}, \mathrm{CH}) ;{ }^{13} \mathrm{C} \mathrm{NMR}$ $\left(\mathrm{CD}_{3} \mathrm{OD}\right) \delta 22.48,52.94,100.83,113.98,121.04,122.16,125.81,126.35,126.93$, 129.41, 133.21, 137.84, 167.54, 173.30; MS (CI) $m / z 259[\mathrm{M}+\mathrm{H}]^{+}$; Anal. Calcd for $\mathrm{C}_{14} \mathrm{H}_{14} \mathrm{~N}_{2} \mathrm{O}_{3}: \mathrm{C}, 65.11 ; \mathrm{H}, 5.46 ; \mathrm{N}, 10.85$. Found: C, 64.75; H, 5.53; N, 10.66.

$(Z)-N^{\alpha}$-acetyl-2,3-dehydro-3-(indol-5'-yl)-alanine, methyl ester (5c). Compound 5c was isolated in $55 \%$ yield by recrystallization from ethyl acetate as a white solid. mp: 187.0-187.9 ${ }^{\circ} \mathrm{C} ;{ }^{1} \mathrm{H}$ NMR $\left(\mathrm{CD}_{3} \mathrm{OD}\right) \delta 2.14\left(\mathrm{~s}, 3 \mathrm{H}, \mathrm{CH}_{3}\right), 3.79\left(\mathrm{~s}, 3 \mathrm{H}, \mathrm{OCH}_{3}\right), 6.49\left(\mathrm{~d},{ }^{3} J_{\mathrm{HH}}\right.$ 
$=3.1 \mathrm{~Hz}, 1 \mathrm{H}, \mathrm{Ar}), 7.26\left(\mathrm{~d},{ }^{3} J_{\mathrm{HH}}=3.2 \mathrm{~Hz}, 1 \mathrm{H}, \mathrm{Ar}\right), 7.40-7.42(\mathrm{~m}, 2 \mathrm{H}, \mathrm{Ar}), 7.61(\mathrm{~s}, 1 \mathrm{H}$, $\mathrm{CH}), 7.85$ (br s, $1 \mathrm{H}, \mathrm{Ar}) ;{ }^{13} \mathrm{C} \mathrm{NMR}\left(\mathrm{CD}_{3} \mathrm{OD}\right) \delta 22.59,52.71,103.30,112.36,123.01$, $124.43,124.51,125.65,126.88,129.55,138.42,138.61,167.73,173.21 ; \mathrm{MS}(\mathrm{CI}) \mathrm{m} / \mathrm{z}$ $259[\mathrm{M}+\mathrm{H}]^{+}$; Anal. Calcd for $\mathrm{C}_{14} \mathrm{H}_{14} \mathrm{~N}_{2} \mathrm{O}_{3}:$ C, 65.11; H, 5.46; N, 10.85. Found: C, 64.83; H, 5.48; N, 10.67.

(Z)- $N^{\alpha}$-acetyl-2,3-dehydro-3-(indol-6'-yl)-alanine, methyl ester (5d). Compound 5d was isolated in 51\% yield by recrystallization from ethyl acetate as a white solid. mp: 169.2-171.3 ${ }^{\circ} \mathrm{C} ;{ }^{1} \mathrm{H}$ NMR $\left(\mathrm{CD}_{3} \mathrm{OD}\right) \delta 2.15\left(\mathrm{~s}, 3 \mathrm{H}, \mathrm{CH}_{3}\right), 3.79\left(\mathrm{~s}, 3 \mathrm{H}, \mathrm{OCH}_{3}\right), 6.46(\mathrm{~s}, 1 \mathrm{H}$, $\operatorname{Ar}), 7.28\left(\mathrm{~d},{ }^{3} J_{\mathrm{HH}}=8.0 \mathrm{~Hz}, 1 \mathrm{H}, \mathrm{Ar}\right), 7.34\left(\mathrm{~d},{ }^{3} J_{\mathrm{HH}}=2.8 \mathrm{~Hz}, 1 \mathrm{H}, \mathrm{Ar}\right), 7.55\left(\mathrm{~d},{ }^{3} J_{\mathrm{HH}}=8.4\right.$ $\mathrm{Hz}, 1 \mathrm{H}, \mathrm{Ar}), 7.59$ (s, 1H, CH), 7.71 (br s, $1 \mathrm{H}, \mathrm{Ar}) ;{ }^{13} \mathrm{C} \mathrm{NMR}\left(\mathrm{CD}_{3} \mathrm{OD}\right) \delta 22.61,52.81$, $102.62,114.57,121.14,122.42,123.43,127.31,128.33,130.93,137.37,138.13,167.45$, 173.23; MS (CI) $m / z 259[\mathrm{M}+\mathrm{H}]^{+}$; Anal. Calcd for $\mathrm{C}_{14} \mathrm{H}_{14} \mathrm{~N}_{2} \mathrm{O}_{3}: \mathrm{C}, 65.11 ; \mathrm{H}, 5.46 ; \mathrm{N}$, 10.85. Found: C, 64.82; H, 5.60; N, 10.94 .

(Z)- $N^{\alpha}$-acetyl- $N^{\text {in }}$-Boc-2,3-dehydro-3-(indol-4'-yl)-alanine, methyl ester $(6 \mathrm{~b})$. Compound $\mathbf{6 b}$ was isolated in $68 \%$ yield by recrystallization from ethyl acetate and flash chromatography of the remaining mother liquor as a yellow solid. $\mathrm{mp}: 150.5-151.8^{\circ} \mathrm{C}$; ${ }^{1} \mathrm{H} \mathrm{NMR}\left(\mathrm{CDCl}_{3}\right) \delta 1.68\left(\mathrm{~s}, 9 \mathrm{H}, \mathrm{CH}_{3}\right), 2.05(\mathrm{~s}, 3 \mathrm{H}, \mathrm{Ac}), 3.88\left(\mathrm{~s}, 3 \mathrm{H}, \mathrm{OCH}_{3}\right), 6.62$ (br s, $1 \mathrm{H}, \mathrm{Ar}), 7.04(\mathrm{br} \mathrm{s}, 1 \mathrm{H}, \mathrm{NH}), 7.27-7.35(\mathrm{~m}, 2 \mathrm{H}, \mathrm{Ar}), 7.58(\mathrm{~s}, 1 \mathrm{H}, \mathrm{Ar}), 7.64\left(\mathrm{~d},{ }^{3} J_{\mathrm{HH}}=3.0\right.$ $\mathrm{Hz}, 1 \mathrm{H}, \mathrm{Ar}), 8.16\left(\mathrm{~d},{ }^{3} J_{\mathrm{HH}}=7.2 \mathrm{~Hz}, 1 \mathrm{H}, \mathrm{Ar}\right) ;{ }^{13} \mathrm{C} \mathrm{NMR}\left(\mathrm{CDCl}_{3}\right) \delta 23.22,28.15,52.68$, $84.05,105.47,116.05,122.63,124.03,125.70,125.90,126.53,128.07,129.76,135.17$, 149.31, 165.43, 168.76; MS (CI) m/z $359[\mathrm{M}+\mathrm{H}]^{+}$; HRMS (FAB) calcd for $\mathrm{C}_{19} \mathrm{H}_{23} \mathrm{~N}_{2} \mathrm{O}_{5}$ $[\mathrm{M}+\mathrm{H}]^{+}$359.1607, found 359.1607. 
$(Z)-N^{\alpha}$-acetyl- $N^{\text {in }}$-Boc-2,3-dehydro-3-(indol-6'-yl)-alanine, methyl ester $(6 \mathrm{~d})$. Compound $\mathbf{6 d}$ was isolated in $46 \%$ yield by flash chromatography (diethyl ether) as a yellow solid. mp: $119.5-121.2{ }^{\circ} \mathrm{C} ;{ }^{1} \mathrm{H}$ NMR $\left(\mathrm{CDCl}_{3}\right) \delta 1.65\left(\mathrm{~s}, 9 \mathrm{H}, \mathrm{CH}_{3}\right), 2.21(\mathrm{~s}, 3 \mathrm{H}$, Ac), $3.86\left(\mathrm{~s}, 3 \mathrm{H}, \mathrm{OCH}_{3}\right), 6.54\left(\mathrm{br} \mathrm{d},{ }^{3} J_{\mathrm{HH}}=3.6 \mathrm{~Hz}, 1 \mathrm{H}, \mathrm{Ar}\right), 7.09(\mathrm{br} \mathrm{s}, 1 \mathrm{H}, \mathrm{NH}), 7.35(\mathrm{~d}$, $\left.{ }^{3} J_{\mathrm{HH}}=8.1 \mathrm{~Hz}, 1 \mathrm{H}, \mathrm{Ar}\right), 7.51-7.61(\mathrm{~m}, 3 \mathrm{H}, \mathrm{Ar}), 8.46(\mathrm{br} \mathrm{s}, 1 \mathrm{H}, \mathrm{Ar}) ;{ }^{13} \mathrm{C} \mathrm{NMR}\left(\mathrm{CDCl}_{3}\right) \delta$ $23.49,28.14,52.58,83.81,107.10,116.57,120.67,122.89,124.79,127.56,129.53$, 131.39, 133.52, 135.26, 149.17, 165.85, 168.78; MS (CI) m/z $359[\mathrm{M}+\mathrm{H}]^{+}$; Anal. Calcd for $\mathrm{C}_{19} \mathrm{H}_{22} \mathrm{~N}_{2} \mathrm{O}_{5}$ : C, 63.68; H, 6.19; N, 7.82. Found: C, 63.83; H, 6.13; N, 7.60.

(Z)- $N^{\alpha}$-Cbz- $N^{\text {in }}$-Boc-2,3-dehydro-3-(indol-4'-yl)-alanine, methyl ester (7b). Compound $\mathbf{7 b}$ was isolated in $83 \%$ yield by flash chromatography (20\% ethyl acetate in hexane) as a yellow soild. mp: $83.4-84.7{ }^{\circ} \mathrm{C} ;{ }^{1} \mathrm{H} \mathrm{NMR}\left(\mathrm{CD}_{3} \mathrm{OD}\right) \delta 1.65\left(\mathrm{~s}, 9 \mathrm{H}, \mathrm{CH}_{3}\right)$, $3.80\left(\mathrm{br} \mathrm{s}, 3 \mathrm{H}, \mathrm{OCH}_{3}\right), 5.07\left(\mathrm{br} \mathrm{s}, 2 \mathrm{H}, \mathrm{CH}_{2}\right), 6.64\left(\mathrm{~d},{ }^{3} J_{\mathrm{HH}}=3.8 \mathrm{~Hz}, 1 \mathrm{H}, \mathrm{Ar}\right), 7.21-7.32$ (m, 6H, Ph, Ar), $7.52\left(\mathrm{br} \mathrm{d},{ }^{3} J_{\mathrm{HH}}=7.5 \mathrm{~Hz}, 1 \mathrm{H}, \mathrm{Ar}\right), 7.57\left(\mathrm{~d},{ }^{3} J_{\mathrm{HH}}=3.8 \mathrm{~Hz}, 1 \mathrm{H}, \mathrm{Ar}\right), 7.64$ $(\mathrm{s}, 1 \mathrm{H}, \mathrm{CH}) 8.11\left(\mathrm{~d},{ }^{3} J_{\mathrm{HH}}=8.3 \mathrm{~Hz}, 1 \mathrm{H}, \mathrm{Ar}\right) ;{ }^{13} \mathrm{C} \mathrm{NMR}\left(\mathrm{CD}_{3} \mathrm{OD}\right) \delta 28.38,53.04,67.92$, $85.16,106.41,116.98,124.03,125.03,126.98,127.20,127.49,128.78,128.88,129.16$, 129.23, 130.67, 131.43, 136.47, 150.56, 156.68, 167.24; MS (CI) $\mathrm{m} / z 451[\mathrm{M}+\mathrm{H}]^{+}$; Anal. Calcd for $\mathrm{C}_{25} \mathrm{H}_{26} \mathrm{~N}_{2} \mathrm{O}_{6}$ : C, 66.66; H, 5.82; N, 6.22. Found: C, 66.78; H, 5.94; N, 6.30 .

(Z)- $N^{\alpha}$-Cbz- $N^{i n}$-Boc-2,3-dehydro-3-(indol-5'-yl)-alanine, methyl ester (7c). Compound $7 \mathbf{c}$ was isolated in $85 \%$ yield by flash chromatography (20\% ethyl acetate in hexane) as a yellow solid. mp: $125.3-126.5^{\circ} \mathrm{C} ;{ }^{1} \mathrm{H}$ NMR $\left(\mathrm{CD}_{3} \mathrm{OD}\right) \delta 1.67\left(\mathrm{~s}, 9 \mathrm{H}, \mathrm{CH}_{3}\right)$, $3.79\left(\mathrm{br} \mathrm{s}, 3 \mathrm{H}, \mathrm{OCH}_{3}\right), 5.15$ (br s, $\left.2 \mathrm{H}, \mathrm{CH}_{2}\right), 6.59\left(\mathrm{~d},{ }^{3} J_{\mathrm{HH}}=3.7 \mathrm{~Hz}, 1 \mathrm{H}, \mathrm{Ar}\right), 7.21-7.45$ $(\mathrm{m}, 5 \mathrm{H}, \mathrm{Ph}), 7.52(\mathrm{~s}, 1 \mathrm{H}, \mathrm{Ar}), 7.57\left(\mathrm{dd},{ }^{3} J_{\mathrm{HH}}=8.9 \mathrm{~Hz},{ }^{4} J_{\mathrm{HH}}=1.5 \mathrm{~Hz}, 1 \mathrm{H}, \mathrm{Ar}\right), 7.64(\mathrm{~d}$, 
$\left.{ }^{3} J_{\mathrm{HH}}=3.7 \mathrm{~Hz}, 1 \mathrm{H}, \mathrm{Ar}\right), 7.85(\mathrm{~s}, 1 \mathrm{H}, \mathrm{CH}), 8.08\left(\mathrm{~d},{ }^{3} J_{\mathrm{HH}}=8.7 \mathrm{~Hz}, 1 \mathrm{H}, \mathrm{Ar}\right) ;{ }^{13} \mathrm{C} \mathrm{NMR}$ $\left(\mathrm{CD}_{3} \mathrm{OD}\right) \delta 28.38,52.90,67.97,85.33,108.56,115.95,124.31,124.94,127.29,127.78$, $128.63,128.75,128.93,129.30,132.04,136.14,136.85,138.02,150.58,157.10,167.55$; MS (CI) $m / z 451[\mathrm{M}+\mathrm{H}]^{+}$; Anal. Calcd for $\mathrm{C}_{25} \mathrm{H}_{26} \mathrm{~N}_{2} \mathrm{O}_{6}$ : C, 66.66; H, 5.82; N, 6.22. Found: C, 66.32; H, 5.74; N, 6.13 .

Ring-closed olefination product of 2a (8). Compound $\mathbf{8}$ was isolated in $59 \%$ yield by flash chromatography $\left(\mathrm{CH}_{2} \mathrm{Cl}_{2}\right)$, and then purified further by recrystallization $\left(\mathrm{CH}_{2} \mathrm{Cl}_{2}\right)$ as a yellow solid. mp: $220.7-221.8{ }^{\circ} \mathrm{C} ;{ }^{1} \mathrm{H} \mathrm{NMR}\left(\mathrm{CDCl}_{3}\right) \delta 3.98(\mathrm{~s}, 3 \mathrm{H}$, $\left.\mathrm{OCH}_{3}\right), 6.88(\mathrm{~s}, 1 \mathrm{H}, \mathrm{Ar}), 7.30\left(\mathrm{~d},{ }^{4} J_{\mathrm{HH}}=1.9 \mathrm{~Hz}, 1 \mathrm{H}, \mathrm{CH}\right), 7.40\left(\mathrm{td},{ }^{3} J_{\mathrm{HH}}=7.4 \mathrm{~Hz},{ }^{4} J_{\mathrm{HH}}=\right.$ $1.0 \mathrm{~Hz}, 1 \mathrm{H}, \mathrm{Ar}), 7.44\left(\mathrm{td},{ }^{3} J_{\mathrm{HH}}=8.1 \mathrm{~Hz},{ }^{4} J_{\mathrm{HH}}=1.2 \mathrm{~Hz}, 1 \mathrm{H}, \mathrm{Ar}\right), 7.70\left(\mathrm{~d},{ }^{3} J_{\mathrm{HH}}=7.6 \mathrm{~Hz}\right.$, $1 \mathrm{H}, \mathrm{Ar}), 8.23(\mathrm{br} \mathrm{s}, 1 \mathrm{H}, \mathrm{NH}), 8.67\left(\mathrm{~d},{ }^{3} J_{\mathrm{HH}}=8.3 \mathrm{~Hz}, 1 \mathrm{H}, \mathrm{Ar}\right) ;{ }^{13} \mathrm{C} \mathrm{NMR}\left(\mathrm{CDCl}_{3}\right) \delta 53.16$, $104.18,104.87,116.24,120.55,124.26,124.46,130.24,133.34,133.88,147.10,161.89$ (12 C found); MS (CI) $m / z 243[\mathrm{M}+\mathrm{H}]^{+}$; HRMS (FAB) calcd for $\mathrm{C}_{13} \mathrm{H}_{11} \mathrm{~N}_{2} \mathrm{O}_{3}[\mathrm{M}+\mathrm{H}]^{+}$ 243.0770, found 243.0772 .

N-Me Ring-closed olefination product of 2a (9). Compound 9 was isolated in $35 \%$ yield by flash chromatography $\left(\mathrm{CH}_{2} \mathrm{Cl}_{2}\right)$, and purified further by recrystallization $\left(\mathrm{CH}_{2} \mathrm{Cl}_{2}\right)$ as a yellow solid. mp: $203.0-204.1{ }^{\circ} \mathrm{C} ;{ }^{1} \mathrm{H} \mathrm{NMR}\left(\mathrm{CDCl}_{3}\right) \delta 3.73(\mathrm{~s}, 3 \mathrm{H}$, $\left.\mathrm{NCH}_{3}\right), 3.92\left(\mathrm{~s}, 3 \mathrm{H}, \mathrm{OCH}_{3}\right), 6.75(\mathrm{~s}, 1 \mathrm{H}, \mathrm{Ar}), 7.27(\mathrm{~s}, 1 \mathrm{H}, \mathrm{CH}), 7.37\left(\mathrm{td},{ }^{3} J_{\mathrm{HH}}=7.2 \mathrm{~Hz}\right.$, $\left.{ }^{4} J_{\mathrm{HH}}=1.5 \mathrm{~Hz}, 1 \mathrm{H}, \mathrm{Ar}\right), 7.42\left(\mathrm{td},{ }^{3} J_{\mathrm{HH}}=7.2 \mathrm{~Hz},{ }^{4} J_{\mathrm{HH}}=1.7 \mathrm{~Hz}, 1 \mathrm{H}, \mathrm{Ar}\right), 7.67\left(\mathrm{~d},{ }^{3} J_{\mathrm{HH}}=7.1\right.$ $\mathrm{Hz}, 1 \mathrm{H}, \mathrm{Ar}), 8.69\left(\mathrm{~d},{ }^{3} J_{\mathrm{HH}}=8.5 \mathrm{~Hz}, 1 \mathrm{H}, \mathrm{Ar}\right) ;{ }^{13} \mathrm{C} \mathrm{NMR}\left(\mathrm{CDCl}_{3}\right) \delta 33.51,52.79,102.46$, $107.15,116.32,120.29,124.02,124.06,129.01,130.23,131.94,133.79,148.83,162.59$; MS (CI) $m / z 257[\mathrm{M}+\mathrm{H}]^{+}$; Anal. Calcd for $\mathrm{C}_{14} \mathrm{H}_{12} \mathrm{~N}_{2} \mathrm{O}_{3}:$ C, 65.62; H, 4.72; N, 10.93 . Found: C, 65.35; H, 4.82; N, 10.85 . 
Ring-closed olefination product of 2e (10). Compound 10 was isolated in $76 \%$ yield by recrystallization $\left(\mathrm{CH}_{2} \mathrm{Cl}_{2}\right)$ as a yellow solid. mp: $134.5-136.0{ }^{\circ} \mathrm{C} ;{ }^{1} \mathrm{H} \mathrm{NMR}\left(\mathrm{CDCl}_{3}\right)$ $\delta 3.89\left(\mathrm{~s}, 3 \mathrm{H}, \mathrm{OCH}_{3}\right), 6.49\left(\mathrm{~d},{ }^{3} J_{\mathrm{HH}}=3.4 \mathrm{~Hz}, 1 \mathrm{H}, \mathrm{Ar}\right), 6.69\left(\mathrm{~d},{ }^{4} J_{\mathrm{HH}}=1.2 \mathrm{~Hz}, 1 \mathrm{H}, \mathrm{CH}\right)$, $6.79\left(\mathrm{~d},{ }^{3} J_{\mathrm{HH}}=7.2 \mathrm{~Hz}, 1 \mathrm{H}, \mathrm{Ar}\right), 7.01\left(\mathrm{t},{ }^{3} J_{\mathrm{HH}}=7.6 \mathrm{~Hz}, 1 \mathrm{H}, \mathrm{Ar}\right), 7.31\left(\mathrm{~d},{ }^{3} J_{\mathrm{HH}}=8.0 \mathrm{~Hz}, 1 \mathrm{H}\right.$, Ar), $7.34($ br s, $1 \mathrm{H}, \mathrm{NH}), 7.74\left(\mathrm{~d},{ }^{3} J_{\mathrm{HH}}=3.6 \mathrm{~Hz}, 1 \mathrm{H}, \mathrm{Ar}\right) ;{ }^{13} \mathrm{C} \mathrm{NMR}\left(\mathrm{CDCl}_{3}\right) \delta 53.50$, $107.38,117.13,120.59,122.47,123.17,123.48,124.67,126.65,131.34,133.03,148.93$, 163.16; MS (CI) $m / z 243[\mathrm{M}+\mathrm{H}]^{+}$; Anal. Calcd for $\mathrm{C}_{13} \mathrm{H}_{10} \mathrm{~N}_{2} \mathrm{O}_{3}: \mathrm{C}, 64.46 ; \mathrm{H}, 4.16 ; \mathrm{N}$, 11.56. Found: C, $64.48 ; \mathrm{H}, 4.25 ; \mathrm{N}, 11.34$.

$(S)-N^{\alpha}$-acetyl-3-(indol-2'-yl)-alanine, methyl ester [(S)-11a]. Hydrogenation was performed in ethyl acetate. HPLC analysis (AD column) indicated 98.6\%ee and 92\% conversion. $[\alpha]^{25}=+34.3^{\circ}(c 0.755, \mathrm{MeOH}) ;{ }^{1} \mathrm{H} \mathrm{NMR}\left(\mathrm{CD}_{3} \mathrm{OD}\right) \delta 1.92\left(\mathrm{~s}, 3 \mathrm{H}, \mathrm{CH}_{3}\right)$, $3.14\left(\mathrm{dd},{ }^{2} J_{\mathrm{HH}}=15.1 \mathrm{~Hz},{ }^{3} J_{\mathrm{HH}}=8.5 \mathrm{~Hz}, 1 \mathrm{H}, \mathrm{CH}_{2}\right), 3.27\left(\mathrm{dd},{ }^{2} J_{\mathrm{HH}}=15.0 \mathrm{~Hz},{ }^{3} J_{\mathrm{HH}}=5.8 \mathrm{~Hz}\right.$, $\left.1 \mathrm{H}, \mathrm{CH}_{2}\right), 3.68\left(\mathrm{~s}, 3 \mathrm{H}, \mathrm{OCH}_{3}\right), 4.79\left(\mathrm{dd},{ }^{3} J_{\mathrm{HH}}=8.6 \mathrm{~Hz}, 5.6 \mathrm{~Hz}, 1 \mathrm{H}, \mathrm{CH}\right), 6.19\left(\mathrm{~d},{ }^{5} J_{\mathrm{HH}}=\right.$ $0.5 \mathrm{~Hz}, 1 \mathrm{H}, \mathrm{Ar}), 6.93\left(\mathrm{td},{ }^{3} J_{\mathrm{HH}}=7.0 \mathrm{~Hz},{ }^{4} J_{\mathrm{HH}}=1.1 \mathrm{~Hz}, 1 \mathrm{H}, \mathrm{Ar}\right), 7.02\left(\mathrm{td},{ }^{3} J_{\mathrm{HH}}=7.1 \mathrm{~Hz}\right.$, $\left.{ }^{4} J_{\mathrm{HH}}=1.2 \mathrm{~Hz}, 1 \mathrm{H}, \mathrm{Ar}\right), 7.27\left(\mathrm{dd},{ }^{3} J_{\mathrm{HH}}=8.0 \mathrm{~Hz},{ }^{4} J_{\mathrm{HH}}=0.7 \mathrm{~Hz}, 1 \mathrm{H}, \mathrm{Ar}\right), 7.41\left(\mathrm{dd},{ }^{3} J_{\mathrm{HH}}=\right.$ $\left.7.6 \mathrm{~Hz},{ }^{4} J_{\mathrm{HH}}=0.8 \mathrm{~Hz}, 1 \mathrm{H}, \mathrm{Ar}\right) ;{ }^{13} \mathrm{C} \mathrm{NMR}\left(\mathrm{CD}_{3} \mathrm{OD}\right) \delta 22.31,31.33,52.80,53.98,101.51$, $111.57,119.93,120.49,121.82,129.86,135.27,137.93,173.11,173.23 ; \mathrm{MS}$ (CI) $\mathrm{m} / \mathrm{z}$ $261[\mathrm{M}+\mathrm{H}]^{+}$; HRMS (FAB) calcd for $\mathrm{C}_{14} \mathrm{H}_{17} \mathrm{~N}_{2} \mathrm{O}_{3}[\mathrm{M}+\mathrm{H}]^{+}$261.1239, found 261.1239.

$(S)-N^{\alpha}$-acetyl-3-(indol-4'-yl)-alanine, methyl ester $[(S)-11 b]$. HPLC analysis (OD column) indicated $99.1 \%$ ee. $[\alpha]^{25}{ }_{\mathrm{D}}=-1.93^{\circ}(c 0.88, \mathrm{MeOH}) ;{ }^{1} \mathrm{H} \mathrm{NMR}\left(\mathrm{CD}_{3} \mathrm{OD}\right) \delta 1.87$ $\left(\mathrm{s}, 3 \mathrm{H}, \mathrm{CH}_{3}\right), 3.20\left(\mathrm{dd},{ }^{2} J_{\mathrm{HH}}=13.7 \mathrm{~Hz},{ }^{3} J_{\mathrm{HH}}=8.3 \mathrm{~Hz}, 1 \mathrm{H}, \mathrm{CH}_{2}\right), 3.40\left(\mathrm{dd},{ }^{2} J_{\mathrm{HH}}=13.7 \mathrm{~Hz}\right.$, $\left.{ }^{3} J_{\mathrm{HH}}=6.0 \mathrm{~Hz}, 1 \mathrm{H}, \mathrm{CH}_{2}\right), 3.62\left(\mathrm{~s}, 3 \mathrm{H}, \mathrm{OCH}_{3}\right), 4.78\left(\mathrm{dd},{ }^{3} J_{\mathrm{HH}}=8.0 \mathrm{~Hz}, 6.2 \mathrm{~Hz}, 1 \mathrm{H}, \mathrm{CH}\right)$, $6.51\left(\mathrm{dd},{ }^{3} \mathrm{~J}_{\mathrm{HH}}=3.2 \mathrm{~Hz},{ }^{5} \mathrm{~J}_{\mathrm{HH}}=0.7 \mathrm{~Hz}, 1 \mathrm{H}, \mathrm{Ar}\right), 6.81\left(\mathrm{~d},{ }^{3} J_{\mathrm{HH}}=7.2 \mathrm{~Hz}, 1 \mathrm{H}, \mathrm{Ar}\right), 7.01(\mathrm{td}$ 
$\left.{ }^{3} J_{\mathrm{HH}}=7.2 \mathrm{~Hz}, 1 \mathrm{H}, \mathrm{Ar}\right), 7.22\left(\mathrm{~d},{ }^{3} J_{\mathrm{HH}}=3.2 \mathrm{~Hz}, 1 \mathrm{H}, \mathrm{Ar}\right), 7.26\left(\mathrm{~d},{ }^{3} J_{\mathrm{HH}}=8.2 \mathrm{~Hz}, 1 \mathrm{H}, \mathrm{Ar}\right)$; ${ }^{13} \mathrm{C}$ NMR $\left(\mathrm{CD}_{3} \mathrm{OD}\right) \delta 22.34,36.47,52.61,54.93,100.20,111.10,120.40,122.06,125.37$, 128.78, 129.10, 137.48, 172.92, 173.77; MS (CI) $m / z, 261[\mathrm{M}+\mathrm{H}]^{+}$; HRMS (FAB) calcd for $\mathrm{C}_{14} \mathrm{H}_{17} \mathrm{~N}_{2} \mathrm{O}_{3}[\mathrm{M}+\mathrm{H}]^{+}$261.1239, found 261.1236.

(S)- $N^{\alpha}$-acetyl-3-(indol-5'-yl)-alanine, methyl ester [(S)-11c]. HPLC analysis (OD column $)$ indicated $98.0 \%$ ee. $[\alpha]_{\mathrm{D}}^{25}=+38.7^{\circ}(c 0.82, \mathrm{MeOH}) ;{ }^{1} \mathrm{H}$ NMR $\left(\mathrm{CD}_{3} \mathrm{OD}\right) \delta 1.88$ $\left(\mathrm{s}, 3 \mathrm{H}, \mathrm{CH}_{3}\right), 3.00\left(\mathrm{dd},{ }^{2} J_{\mathrm{HH}}=13.8 \mathrm{~Hz},{ }^{3} J_{\mathrm{HH}}=8.6 \mathrm{~Hz}, 1 \mathrm{H}, \mathrm{CH}_{2}\right), 3.18\left(\mathrm{dd},{ }^{2} J_{\mathrm{HH}}=13.8 \mathrm{~Hz}\right.$, $\left.{ }^{3} J_{\mathrm{HH}}=5.9 \mathrm{~Hz}, 1 \mathrm{H}, \mathrm{CH}_{2}\right), 3.65\left(\mathrm{~s}, 3 \mathrm{H}, \mathrm{OCH}_{3}\right), 4.65\left(\mathrm{dd},{ }^{3} J_{\mathrm{HH}}=8.5 \mathrm{~Hz}, 5.9 \mathrm{~Hz}, 1 \mathrm{H}, \mathrm{CH}\right)$, $6.37\left(\mathrm{dd},{ }^{3} \mathrm{~J}_{\mathrm{HH}}=3.2 \mathrm{~Hz},{ }^{5} \mathrm{~J}_{\mathrm{HH}}=0.9 \mathrm{~Hz}, 1 \mathrm{H}, \mathrm{Ar}\right), 6.93\left(\mathrm{dd},{ }^{3} J_{\mathrm{HH}}=8.3 \mathrm{~Hz},{ }^{4} J_{\mathrm{HH}}=1.6 \mathrm{~Hz}\right.$, $1 \mathrm{H}, \mathrm{Ar}), 7.18\left(\mathrm{~d},{ }^{3} J_{\mathrm{HH}}=2.9 \mathrm{~Hz}, 1 \mathrm{H}, \mathrm{Ar}\right), 7.29\left(\mathrm{~d},{ }^{3} J_{\mathrm{HH}}=8.3 \mathrm{~Hz}, 1 \mathrm{H}, \mathrm{Ar}\right), 7.35\left(\mathrm{~d},{ }^{4} J_{\mathrm{HH}}=\right.$ $0.8 \mathrm{~Hz}, 1 \mathrm{H}, \mathrm{Ar}) ;{ }^{13} \mathrm{C} \mathrm{NMR}\left(\mathrm{CD}_{3} \mathrm{OD}\right) \delta 22.28,38.79,52.56,56.11,101.99,111.99$, $121.39,123.28,125.78,128.02,129.47,136.58,172.94,173.76$; MS (CI) $m / z 261[\mathrm{M}+$ $\mathrm{H}]^{+}$; HRMS (FAB) calcd for $\mathrm{C}_{14} \mathrm{H}_{17} \mathrm{~N}_{2} \mathrm{O}_{3}[\mathrm{M}+\mathrm{H}]^{+}$261.1239, found 261.1236.

$(S)$ - $N^{\alpha}$-acetyl-3-(indol-6'-yl)-alanine, methyl ester [(S)-11d]. HPLC analysis (AD column) indicated $96.7 \%$ ee. $[\alpha]_{\mathrm{D}}^{25}=+28.6^{\circ}(c 1.13, \mathrm{MeOH}) ;{ }^{1} \mathrm{H}$ NMR $\left(\mathrm{CD}_{3} \mathrm{OD}\right) \delta 1.89$ $\left(\mathrm{s}, 3 \mathrm{H}, \mathrm{CH}_{3}\right), 3.03\left(\mathrm{dd},{ }^{2} J_{\mathrm{HH}}=13.8 \mathrm{~Hz},{ }^{3} J_{\mathrm{HH}}=8.4 \mathrm{~Hz}, 1 \mathrm{H}, \mathrm{CH}_{2}\right), 3.19\left(\mathrm{dd},{ }^{2} J_{\mathrm{HH}}=13.8 \mathrm{~Hz}\right.$, $\left.{ }^{3} J_{\mathrm{HH}}=5.9 \mathrm{~Hz}, 1 \mathrm{H}, \mathrm{CH}_{2}\right), 3.65\left(\mathrm{~s}, 3 \mathrm{H}, \mathrm{OCH}_{3}\right), 4.67\left(\mathrm{dd},{ }^{3} J_{\mathrm{HH}}=8.4 \mathrm{~Hz}, 5.9 \mathrm{~Hz}, 1 \mathrm{H}, \mathrm{CH}\right)$, $6.37\left(\mathrm{~d},{ }^{3} \mathrm{~J}_{\mathrm{HH}}=3.1 \mathrm{~Hz}{ }^{5} \mathrm{~J}_{\mathrm{HH}}=0.9 \mathrm{~Hz}, 1 \mathrm{H}, \mathrm{Ar}\right), 6.84\left(\mathrm{dd},{ }^{3} J_{\mathrm{HH}}=8.1 \mathrm{~Hz},{ }^{4} J_{\mathrm{HH}}=1.4 \mathrm{~Hz}, 1 \mathrm{H}\right.$, $\mathrm{Ar}), 7.16\left(\mathrm{~d},{ }^{3} J_{\mathrm{HH}}=3.2 \mathrm{~Hz}, 1 \mathrm{H}, \mathrm{Ar}\right), 7.20(\mathrm{br} \mathrm{s}, 1 \mathrm{H}, \mathrm{Ar}), 7.44\left(\mathrm{~d},{ }^{3} J_{\mathrm{HH}}=8.1 \mathrm{~Hz}, 1 \mathrm{H}, \mathrm{Ar}\right)$; ${ }^{13} \mathrm{C}$ NMR $\left(\mathrm{CD}_{3} \mathrm{OD}\right) \delta 22.30,38.88,52.27,55.94,102.02,112.36,120.97,121.29,125.48$, 128.26, 130.57, 137.66, 172.92, 173.65; MS (CI) m/z $261[\mathrm{M}+\mathrm{H}]^{+}$; HRMS (FAB) calcd for $\mathrm{C}_{14} \mathrm{H}_{17} \mathrm{~N}_{2} \mathrm{O}_{3}[\mathrm{M}+\mathrm{H}]^{+}$261.1239, found 261.1237. 
$(S)-N^{\alpha}$-acetyl- $N^{\text {in }}$ Boc-3-(indol-4'-yl)-alanine, methyl ester [(S)-12b]. HPLC analysis OD column) indicated $98.9 \%$ ee. $[\alpha]_{\mathrm{D}}^{25}=+11.0^{\circ}(c 0.89, \mathrm{MeOH}) ;{ }^{1} \mathrm{H}$ NMR $\left(\mathrm{CD}_{3} \mathrm{OD}\right) \delta$ $1.66\left(\mathrm{~s}, 9 \mathrm{H}, \mathrm{CH}_{3}\right), 1.87\left(\mathrm{~s}, 3 \mathrm{H}, \mathrm{CH}_{3}\right), 3.20\left(\mathrm{dd},{ }^{2} J_{\mathrm{HH}}=13.8 \mathrm{~Hz},{ }^{3} J_{\mathrm{HH}}=8.3 \mathrm{~Hz}, 1 \mathrm{H}, \mathrm{CH}_{2}\right)$, $3.38\left(\mathrm{dd},{ }^{2} J_{\mathrm{HH}}=13.9 \mathrm{~Hz}, 6.2 \mathrm{~Hz}, 1 \mathrm{H}, \mathrm{CH}_{2}\right), 3.64\left(\mathrm{~s}, 3 \mathrm{H}, \mathrm{OCH}_{3}\right), 4.60\left(\mathrm{dd},{ }^{3} J_{\mathrm{HH}}=8.2 \mathrm{~Hz}\right.$, $6.2 \mathrm{~Hz}, 1 \mathrm{H}, \mathrm{CH}), 6.70\left(\mathrm{~d},{ }^{3} J_{\mathrm{HH}}=3.8 \mathrm{~Hz}, 1 \mathrm{H}, \mathrm{Ar}\right), 7.03\left(\mathrm{~d},{ }^{3} J_{\mathrm{HH}}=7.3 \mathrm{~Hz}, 1 \mathrm{H}, \mathrm{Ar}\right), 7.21(\mathrm{t}$ $\left.{ }^{3} J_{\mathrm{HH}}=7.9 \mathrm{~Hz}, 1 \mathrm{H}, \mathrm{Ar}\right), 7.62\left(\mathrm{~d},{ }^{3} J_{\mathrm{HH}}=3.8 \mathrm{~Hz}, 1 \mathrm{H}, \mathrm{Ar}\right), 8.02\left(\mathrm{~d}^{3} J_{\mathrm{HH}}=8.3 \mathrm{~Hz}, 1 \mathrm{H}, \mathrm{Ar}\right) ;{ }^{13} \mathrm{C}$ $\mathrm{NMR}\left(\mathrm{CD}_{3} \mathrm{OD}\right) \delta 22.32,28.40,35.86,52.70,54.91,84.92,106.18,114.76,124.28$, $125.09,126.67,130.29,131.29,136.44,150.80,172.86,173.29 ; \mathrm{MS}(\mathrm{CI}) \mathrm{m} / z .361[\mathrm{M}+$ $\mathrm{H}]^{+}$; HRMS (FAB) calcd for $\mathrm{C}_{19} \mathrm{H}_{25} \mathrm{~N}_{2} \mathrm{O}_{5}[\mathrm{M}+\mathrm{H}]^{+}$361.1763, found 361.1768.

$(S)-N^{\alpha}$-acetyl- $N^{\text {in }}$ Boc-3-(indol-6'-yl)-alanine, methyl ester $[(S)-12 d]$. HPLC analysis (OD column) indicated $98.5 \%$ ee. $[\alpha]^{25}=+23.7^{\circ}(c 1.10, \mathrm{MeOH}) ;{ }^{1} \mathrm{H} \mathrm{NMR}\left(\mathrm{CD}_{3} \mathrm{OD}\right) \delta$ $1.66\left(\mathrm{~s}, 9 \mathrm{H}, \mathrm{CH}_{3}\right), 1.91\left(\mathrm{~s}, 3 \mathrm{H}, \mathrm{CH}_{3}\right), 3.07\left(\mathrm{dd},{ }^{2} J_{\mathrm{HH}}=13.8 \mathrm{~Hz},{ }^{3} J_{\mathrm{HH}}=8.6 \mathrm{~Hz}, 1 \mathrm{H}, \mathrm{CH}_{2}\right)$, $3.23\left(\mathrm{dd},{ }^{2} J_{\mathrm{HH}}=13.5 \mathrm{~Hz}, 6.0 \mathrm{~Hz}, 1 \mathrm{H}, \mathrm{CH}_{2}\right), 3.68\left(\mathrm{~s}, 3 \mathrm{H}, \mathrm{OCH}_{3}\right), 4.69\left(\mathrm{dd},{ }^{3} J_{\mathrm{HH}}=8.2 \mathrm{~Hz}\right.$, $6.2 \mathrm{~Hz}, 1 \mathrm{H}, \mathrm{CH}), 6.55\left(\mathrm{~d},{ }^{3} J_{\mathrm{HH}}=3.6 \mathrm{~Hz}, 1 \mathrm{H}, \mathrm{Ar}\right), 7.05\left(\mathrm{dd},{ }^{3} J_{\mathrm{HH}}=8.0 \mathrm{~Hz},{ }^{4} J_{\mathrm{HH}}=1.2 \mathrm{~Hz}\right.$, $1 \mathrm{H}, \mathrm{Ar}), 7.46\left(\mathrm{~d}^{3} J_{\mathrm{HH}}=8.0 \mathrm{~Hz}, 1 \mathrm{H}, \mathrm{Ar}\right), 7.54\left(\mathrm{~d},{ }^{3} J_{\mathrm{HH}}=4.0 \mathrm{~Hz}, 1 \mathrm{H}, \mathrm{Ar}\right), 8.00(\mathrm{br} \mathrm{s}, 1 \mathrm{H}$, $\mathrm{Ar}) ;{ }^{13} \mathrm{C} \mathrm{NMR}\left(\mathrm{CD}_{3} \mathrm{OD}\right) \delta 22.33,28.41,39.00,52.66,55.78,84.83,108.10,116.48$, $121.71,124.90,126.72,130.74,134.05,136.63,150.81,172.89,173.33 ; \mathrm{MS}$ (CI) $\mathrm{m} / \mathrm{z}$ $361[\mathrm{M}+\mathrm{H}]^{+}$; HRMS (FAB) calcd for $\mathrm{C}_{19} \mathrm{H}_{25} \mathrm{~N}_{2} \mathrm{O}_{5}[\mathrm{M}+\mathrm{H}]^{+}$361.1763, found 361.1754

$(S)-N^{\alpha}-C b z-N^{\text {in }}$ Boc-3-(indol-4'-yl)-alanine, methyl ester $[(S)-13 b]$. The $(S, S)-$ MeDuPhos catalyst was used. HPLC analysis (OD column) indicated $98.9 \%$ ee. $[\alpha]_{D}^{25}=$ $-13.4^{\circ}(c 0.685, \mathrm{MeOH}) ;{ }^{1} \mathrm{H} \mathrm{NMR}\left(\mathrm{CD}_{3} \mathrm{OD}\right) \delta 1.66\left(\mathrm{~s}, 9 \mathrm{H}, \mathrm{CH}_{3}\right), 3.18\left(\mathrm{dd},{ }^{2} J_{\mathrm{HH}}=13.9\right.$ $\left.\mathrm{Hz},{ }^{3} J_{\mathrm{HH}}=8.7 \mathrm{~Hz}, 1 \mathrm{H}, \mathrm{CH}_{2}\right), 3.38\left(\mathrm{dd},{ }^{2} J_{\mathrm{HH}}=13.8 \mathrm{~Hz},{ }^{3} J_{\mathrm{HH}}=5.7 \mathrm{~Hz}, 1 \mathrm{H}, \mathrm{CH}_{2}\right), 3.65(\mathrm{~s}$, $\left.3 \mathrm{H}, \mathrm{OCH}_{3}\right), 4.54\left(\mathrm{dd},{ }^{3} J_{\mathrm{HH}}=8.8 \mathrm{~Hz}, 5.7 \mathrm{~Hz}, 1 \mathrm{H}, \mathrm{CH}\right), 4.95\left(\mathrm{~d},{ }^{2} J_{\mathrm{HH}}=12.6 \mathrm{~Hz}, 1 \mathrm{H}, \mathrm{CH}_{2}\right)$, 
$5.01\left(\mathrm{~d},{ }^{2} \mathrm{~J}_{\mathrm{HH}}=12.5 \mathrm{~Hz}, 1 \mathrm{H}, \mathrm{CH}_{2}\right), 6.68\left(\mathrm{~d},{ }^{3} J_{\mathrm{HH}}=3.8 \mathrm{~Hz}, 1 \mathrm{H}, \mathrm{Ar}\right), 7.02\left(\mathrm{~d},{ }^{3} J_{\mathrm{HH}}=7.3 \mathrm{~Hz}\right.$, $1 \mathrm{H}, \mathrm{Ar}), 7.19\left(\mathrm{t},{ }^{3} J_{\mathrm{HH}}=7.9 \mathrm{~Hz}, 1 \mathrm{H}, \mathrm{Ar}\right), 7.20-7.33(\mathrm{~m}, 5 \mathrm{H}, \mathrm{Ph}), 7.57\left(\mathrm{~d},{ }^{3} J_{\mathrm{HH}}=3.8 \mathrm{~Hz}\right.$, $1 \mathrm{H}, \mathrm{Ar}), 8.01\left(\mathrm{~d},{ }^{3} J_{\mathrm{HH}}=8.3 \mathrm{~Hz}, 1 \mathrm{H}, \mathrm{Ar}\right) ;{ }^{13} \mathrm{C} \mathrm{NMR}\left(\mathrm{CD}_{3} \mathrm{OD}\right) \delta 28.41,36.05,52.73,56.54$, $84.88,106.23,114.71,124.43,125.09,126.64,128.47,128.73,129.21,130.41,131.21$, 136.42, 137.97, 150.81, 158.02, 173.62; MS (CI) m/z 453 [M + H] ; HRMS (FAB) calcd for $\mathrm{C}_{25} \mathrm{H}_{29} \mathrm{~N}_{2} \mathrm{O}_{6}[\mathrm{M}+\mathrm{H}]^{+}$453.2026, found 453.2033.

$(R)-N^{\alpha}-$ Cbz- $N^{\text {in } B o c-3-(i n d o l-5 '-y l)-a l a n i n e, ~ m e t h y l ~ e s t e r ~}[(R)-13 c]$. The $(R, R)$ MeDuPhos catalyst and $\mathrm{CH}_{2} \mathrm{Cl}_{2}$ solvent were used. HPLC analysis (AD column) indicated $97.5 \%$ ee. $[\alpha]^{25}{ }_{\mathrm{D}}=+6.98^{\circ}(c 0.86, \mathrm{MeOH}) ;{ }^{1} \mathrm{H} \mathrm{NMR}\left(\mathrm{CD}_{3} \mathrm{OD}\right) \delta 1.65(\mathrm{~s}, 9 \mathrm{H}$, $\left.\mathrm{CH}_{3}\right), 2.99\left(\mathrm{dd},{ }^{2} J_{\mathrm{HH}}=13.7 \mathrm{~Hz},{ }^{3} J_{\mathrm{HH}}=9.3 \mathrm{~Hz}, 1 \mathrm{H}, \mathrm{CH}_{2}\right), 3.22\left(\mathrm{dd},{ }^{2} J_{\mathrm{HH}}=13.8 \mathrm{~Hz},{ }^{3} J_{\mathrm{HH}}=\right.$ $\left.5.4 \mathrm{~Hz}, 1 \mathrm{H}, \mathrm{CH}_{2}\right), 3.68\left(\mathrm{~s}, 3 \mathrm{H}, \mathrm{OCH}_{3}\right), 4.48\left(\mathrm{dd},{ }^{3} J_{\mathrm{HH}}=9.1 \mathrm{~Hz}, 5.5 \mathrm{~Hz}, 1 \mathrm{H}, \mathrm{CH}\right), 4.95(\mathrm{~d}$, $\left.{ }^{2} J_{\mathrm{HH}}=12.6 \mathrm{~Hz}, 1 \mathrm{H}, \mathrm{CH}_{2}\right), 5.02\left(\mathrm{~d},{ }^{2} \mathrm{~J}_{\mathrm{HH}}=12.6 \mathrm{~Hz}, 1 \mathrm{H}, \mathrm{CH}_{2}\right), 6.52\left(\mathrm{~d},{ }^{3} J_{\mathrm{HH}}=3.8 \mathrm{~Hz}, 1 \mathrm{H}\right.$, Ar), $7.12\left(\mathrm{dd},{ }^{3} J_{\mathrm{HH}}=8.6 \mathrm{~Hz},{ }^{4} J_{\mathrm{HH}}=1.4 \mathrm{~Hz}, 1 \mathrm{H}, \mathrm{Ar}\right), 7.19-7.28(\mathrm{~m}, 5 \mathrm{H}, \mathrm{Ph}), 7.38(\mathrm{br} \mathrm{s}, 1 \mathrm{H}$ Ar), $7.57\left(\mathrm{~d},{ }^{3} J_{\mathrm{HH}}=3.7 \mathrm{~Hz}, 1 \mathrm{H}, \mathrm{Ar}\right), 8.00\left(\mathrm{~d},{ }^{3} J_{\mathrm{HH}}=8.5 \mathrm{~Hz}, 1 \mathrm{H}, \mathrm{Ar}\right) ;{ }^{13} \mathrm{C} \mathrm{NMR}\left(\mathrm{CD}_{3} \mathrm{OD}\right) \delta$ $28.41,38.60,52.68,57.27,67.42,84.81,108.21,115.76,122.36,126.34,126.96,128.38$, $128.68,129.19,132.07,132.43,135.38,138.00,150.85,158.10,173.77 ;$ MS (CI) $\mathrm{m} / \mathrm{z}$ $453[\mathrm{M}+\mathrm{H}]^{+}$; HRMS (FAB) calcd for $\mathrm{C}_{25} \mathrm{H}_{29} \mathrm{~N}_{2} \mathrm{O}_{6}[\mathrm{M}+\mathrm{H}]^{+}$453.2026, found 453.2034.

The enantiomers of $\mathbf{1 3 d}$ could not be resolved with any of the chiral stationary phases tried. Thus $\mathbf{1 3 d}$ was transformed to the $N^{\alpha}$-Ac- $N^{i n}$-Boc-2', $3^{\prime}$-dihydro-derivative $\mathbf{2 2}$ as follows:

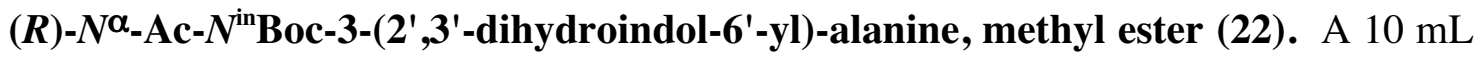
round bottomed flask was charged with $(R)-\mathbf{1 3 d}(5 \mathrm{mg}, 0.01 \mathrm{mmol}), \mathrm{Pd} / \mathrm{C}(10 \mathrm{mg})$. $\mathrm{MeOH}(4 \mathrm{~mL})$ and acetic anhydride $(1 \mathrm{~mL})$ was then added. It was then charged with $\mathrm{H}_{2}$ 
at $1 \mathrm{~atm}$, and stirred at room temperature overnight. The mixture was then filtered, concentrated in vacuo, dissolved in ethyl acetate, and passed through a short plug of $\mathrm{SiO}_{2}$ before HPLC analysis (OD column), which indicated $96.9 \%$ ee. ${ }^{1} \mathrm{H}$ NMR $\left(\mathrm{CD}_{3} \mathrm{OD}\right) \delta$ $1.55\left(\right.$ br s, $\left.9 \mathrm{H}, \mathrm{CH}_{3}\right), 1.91\left(\mathrm{~s}, 3 \mathrm{H}, \mathrm{CH}_{3}\right), 2.91\left(\mathrm{dd},{ }^{2} J_{\mathrm{HH}}=13.6 \mathrm{~Hz},{ }^{3} J_{\mathrm{HH}}=8.4 \mathrm{~Hz}, 1 \mathrm{H}, \mathrm{CH}_{2}\right)$, $3.01\left(\mathrm{t},{ }^{3} J_{\mathrm{HH}}=8.6 \mathrm{~Hz}, 2 \mathrm{H}, \mathrm{CH}_{2}\right), 3.07\left(\mathrm{dd},{ }^{2} J_{\mathrm{HH}}=13.8 \mathrm{~Hz},{ }^{3} J_{\mathrm{HH}}=6.0 \mathrm{~Hz}, 1 \mathrm{H}, \mathrm{CH}_{2}\right), 3.26$ $\left(\mathrm{s}, 3 \mathrm{H}, \mathrm{OCH}_{3}\right), 3.92\left(\mathrm{t},{ }^{3} J_{\mathrm{HH}}=8.6 \mathrm{~Hz}, 2 \mathrm{H}, \mathrm{CH}_{2}\right), 4.62\left(\mathrm{dd},{ }^{3} J_{\mathrm{HH}}=8.5 \mathrm{~Hz}, 5.7 \mathrm{~Hz}, 1 \mathrm{H}\right.$, $\mathrm{CH}), 6.76\left(\mathrm{dd},{ }^{3} J_{\mathrm{HH}}=7.6 \mathrm{~Hz},{ }^{4} J_{\mathrm{HH}}=1.6 \mathrm{~Hz}, 1 \mathrm{H}, \mathrm{Ar}\right), 7.06\left(\mathrm{~d},{ }^{3} J_{\mathrm{HH}}=7.6 \mathrm{~Hz}, 1 \mathrm{H}, \mathrm{Ar}\right), 7.62$ (br s, $1 \mathrm{H}, \mathrm{Ar}) ;{ }^{13} \mathrm{C} \mathrm{NMR}\left(\mathrm{CDCl}_{3}\right) \delta 23.11,27.03,28.37,37.91,47.77,52.30,53.21$, $80.34,115.52,122.80,124.51,129.85,135.04,143.37,152.36,169.55,172.07$; MS (CI) $m / z 363[\mathrm{M}+\mathrm{H}]^{+}$; HRMS (FAB) calcd for $\mathrm{C}_{19} \mathrm{H}_{27} \mathrm{~N}_{2} \mathrm{O}_{5}[\mathrm{M}+\mathrm{H}]^{+}$363.1920, found 363.1914.

Table S1. Summary of Experimental Details for HPLC Enantiomeric Excess Determination.

\begin{tabular}{|c|c|c|c|c|c|}
\hline $\begin{array}{l}\text { amino } \\
\text { acid }\end{array}$ & $\begin{array}{l}\text { Daicel } \\
\text { column } \\
\text { used }\end{array}$ & 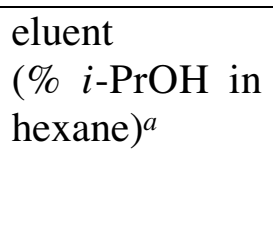 & $\begin{array}{l}\text { retention time } \\
\text { of major }{ }^{b}(S) \\
\text { enantiomer } \\
(\mathrm{min})\end{array}$ & $\begin{array}{l}\text { retention time } \\
\text { of } \text { minor }^{b}(R) \\
\text { enantiomer } \\
(\min )\end{array}$ & $\begin{array}{l}\text { retention times } \\
\text { of } \\
\text { overhydrogen- } \\
\text { ation products } \\
(\mathrm{min})^{c}\end{array}$ \\
\hline $11 a$ & $\mathrm{AD}$ & gradient $^{d}$ & 15.0 & 12.6 & 11.7 \\
\hline 11b & OD & 20 & 23.6 & 14.0 & nd \\
\hline 11c & OD & 15 & 26.0 & 18.7 & nd \\
\hline 11d & $\mathrm{AD}$ & 20 & 10.1 & 7.6 & nd \\
\hline 11e & OD & 10 & 16.5 & 12.9 & 21.6 \\
\hline 12b & OD & 5 & 22.7 & 17.6 & $30.7,42.4$ \\
\hline $12 \mathrm{c}$ & OD & 10 & 13.9 & 11.1 & $10.1,13.8$ \\
\hline 12d & OD & 5 & 17.4 & 14.3 & 20.2 \\
\hline
\end{tabular}




$\begin{array}{cccccc}\text { 13b } & \text { OD } & 5 & 21.8 & 24.0 & 36.1 \\ \text { 13c } & \text { AD } & 10 & 18.8 & 23.3 & 26.8 \\ \text { 13d } & \text { OD } & 5 & 30.4^{\mathrm{e}} & 20.3^{\mathrm{e}} & \text { na }\end{array}$

${ }^{a}$ Flow rate: $1 \mathrm{~mL} / \mathrm{min} . \quad{ }^{b} \mathrm{In}$ reactions using $(S, S)$-DuPhos ligands. $c$ Overhydrogenation products were observed at elevated pressures, or in the case of $\mathbf{1 2}$ and 13, when methanol was used as the hydrogenation solvent. nd: not detected; na: not applicable (see procedure). ${ }^{d}$ Gradient conditions: 0 min: 10\%; 15 min: 15\%; 20 min: 50\%; 30 min: $50 \%$. ${ }^{e} N^{\alpha}-\mathrm{Ac}-N^{\text {in }}-$ Boc-2' $3^{\prime}$-dihydro derivative of $\mathbf{1 3 d}$.

Table S2. List of ligands and complexes in calculations.

\begin{tabular}{lccc}
\hline & Ligand & $\eta^{6}$ Complex & $\eta^{5}$ Complex \\
\hline Indole & $\mathbf{1 4 a}$ & $\mathbf{1 4 b}$ & $\mathbf{1 4 c}$ \\
$N$-Me indole & $\mathbf{1 5 a}$ & $\mathbf{1 5 b}$ & $\mathbf{1 5 c}$ \\
2-Me indole & $\mathbf{1 6 a}$ & $\mathbf{1 6 b}$ & $\mathbf{1 6}$ \\
3-Me indole & $\mathbf{1 7 a}$ & $\mathbf{1 7 b}$ & $\mathbf{1 7} \mathbf{c}$ \\
$4-$ Me indole & $\mathbf{1 8 a}$ & $\mathbf{1 8 b}$ & $\mathbf{1 8 c}$ \\
$5-$ Me indole & $\mathbf{1 9 a}$ & $\mathbf{1 9 b}$ & $\mathbf{1 9 c}$ \\
6-Me indole & $\mathbf{2 0 a}$ & $\mathbf{2 0 b}$ & $\mathbf{2 0 c}$ \\
7-Me indole & $\mathbf{2 1 a}$ & $\mathbf{2 1 b}$ & $\mathbf{2 1 c}$
\end{tabular}

Table S3. Energies for compounds 14-21.

\begin{tabular}{lccc}
\hline & $\begin{array}{c}\text { 6-31G** Absolute } \\
\text { Energy (a.u.) }\end{array}$ & $\begin{array}{c}\text { Zero Point Vibrational } \\
\text { Energy (ZPVE) }(\mathrm{kcal} / \mathrm{mol})^{b}\end{array}$ & $\begin{array}{c}\text { ZPVE Corrected } \\
\text { Absolute Energy (a.u.) }\end{array}$ \\
\hline $\mathbf{N a}^{+}$ & -161.6592884 & N/A & -161.6592884 \\
$\mathbf{1 4 a}$ & -361.4829178 & 78.3815 & -361.3579991 \\
$\mathbf{1 4 b}$ & -523.1941831 & 79.2969 & -523.0678056 \\
$\mathbf{1 4 c}$ & -523.1884604 & 79.1629 & -523.0622964 \\
$\mathbf{1 5 a}$ & -400.5146122 & 95.1479 & -400.3629723
\end{tabular}




\begin{tabular}{|c|c|c|c|}
\hline $15 b$ & -562.2275917 & 95.9716 & -562.0746391 \\
\hline $15 \mathrm{c}$ & -562.2216668 & 95.7873 & -562.0690080 \\
\hline $16 \mathbf{a}$ & -400.5276780 & 94.8350 & -400.3765368 \\
\hline $16 b$ & -562.2415446 & 95.7351 & -562.0889689 \\
\hline $16 \mathrm{c}$ & -562.2351562 & 95.6002 & -562.0827955 \\
\hline $17 \mathbf{a}$ & -400.5242550 & 94.9196 & -400.3729791 \\
\hline $17 b$ & -562.2364210 & 95.8421 & -562.0836748 \\
\hline $17 \mathrm{c}$ & -562.2312926 & 95.7846 & -562.0786381 \\
\hline $18 \mathbf{a}$ & -400.5239258 & 94.9231 & -400.3726442 \\
\hline $18 b$ & -562.2366583 & 95.8430 & -562.0839107 \\
\hline $18 c$ & -562.2306768 & 95.6713 & -562.0782029 \\
\hline 19a & -400.5221746 & 94.7919 & -400.3711022 \\
\hline $19 b$ & -562.2346701 & 95.7342 & -562.0820959 \\
\hline $19 \mathrm{c}$ & -562.2296737 & 95.5975 & -562.0773173 \\
\hline $20 a$ & -400.5228110 & 94.8027 & -400.3717214 \\
\hline $20 b$ & -562.2353168 & 95.7396 & -562.0827340 \\
\hline $20 \mathrm{c}$ & -562.2304897 & 95.6002 & -562.0781290 \\
\hline $21 a$ & -400.5236713 & 94.9240 & -400.3723883 \\
\hline $21 b$ & -562.2365291 & 95.8385 & -562.0837886 \\
\hline 21c & -562.2304208 & 95.6650 & -562.0779569 \\
\hline
\end{tabular}

${ }^{a} \mathrm{HF} / 6-31 \mathrm{G} * * / / \mathrm{HF} / 6-31 \mathrm{G}^{* *}{ }^{b}{ }^{b}$ Scaled by 0.8992.

\section{PDB files for compounds 14-21.}

\section{4a. Indole (LCH0004)}

\begin{tabular}{|c|c|c|c|c|c|c|c|}
\hline \multicolumn{8}{|l|}{ HEADER } \\
\hline REMARK & Spar & & 02 & ported & Molecule00 & & \\
\hline HETATM & 1 & $\mathrm{C}$ & UNK & 0001 & 0.293 & -0.678 & 0.000 \\
\hline НЕTATM & 2 & $\mathrm{C}$ & UNK & 0001 & -2.078 & 0.709 & 0.000 \\
\hline НЕTАТМ & 3 & $\mathrm{C}$ & UNK & 0001 & 0.312 & 0.722 & 0.000 \\
\hline
\end{tabular}




\begin{tabular}{|c|c|c|c|c|c|c|c|}
\hline HETATM & 4 & C & UNK & 0001 & -0.897 & -1.404 & 0.000 \\
\hline HETATM & 5 & $\mathrm{C}$ & UNK & 0001 & -2.074 & -0.695 & 0.000 \\
\hline НЕТАТМ & 6 & $\mathrm{C}$ & UNK & 0001 & -0.901 & 1.418 & 0.000 \\
\hline HETATM & 7 & $\mathrm{H}$ & UNK & 0001 & -0.896 & -2.481 & 0.000 \\
\hline НЕTATM & 8 & $\mathrm{H}$ & UNK & 0001 & -3.011 & -1.224 & 0.000 \\
\hline HETATM & 9 & $\mathrm{H}$ & UNK & 0001 & -0.911 & 2.495 & 0.000 \\
\hline НЕTATM & 10 & $\mathrm{H}$ & UNK & 0001 & -3.019 & 1.232 & 0.000 \\
\hline НЕТАТМ & 11 & $\mathrm{C}$ & UNK & 0001 & 1.698 & 1.118 & 0.000 \\
\hline HETATM & 12 & $\mathrm{H}$ & UNK & 0001 & 2.086 & 2.116 & 0.000 \\
\hline HETATM & 13 & $\mathrm{C}$ & UNK & 0001 & 2.425 & -0.017 & 0.000 \\
\hline HETATM & 14 & $\mathrm{H}$ & UNK & 0001 & 3.489 & -0.146 & 0.000 \\
\hline НЕТАТМ & 15 & $\mathrm{~N}$ & UNK & 0001 & 1.594 & -1.109 & 0.000 \\
\hline НЕТАТМ & 16 & $\mathrm{H}$ & UNK & 0001 & 1.890 & -2.055 & 0.000 \\
\hline CONECT & 1 & 4 & 3 & 15 & & & \\
\hline CONECT & 2 & 5 & 6 & 10 & & & \\
\hline CONECT & 3 & 6 & 1 & 11 & & & \\
\hline CONECT & 4 & 1 & 5 & 7 & & & \\
\hline CONECT & 5 & 4 & 2 & 8 & & & \\
\hline CONECT & 6 & 2 & 3 & 9 & & & \\
\hline CONECT & 7 & 4 & & & & & \\
\hline CONECT & 8 & 5 & & & & & \\
\hline CONECT & 9 & 6 & & & & & \\
\hline CONECT & 10 & 2 & & & & & \\
\hline CONECT & 11 & 12 & 3 & 13 & & & \\
\hline CONECT & 12 & 11 & & & & & \\
\hline CONECT & 13 & 14 & 11 & 15 & & & \\
\hline CONECT & 14 & 13 & & & & & \\
\hline CONECT & 15 & 16 & 13 & 1 & & & \\
\hline CONECT & 16 & 15 & & & & & \\
\hline END & & & & & & & \\
\hline
\end{tabular}

14b. $\eta^{6}$-Indole $\bullet \mathrm{Na}^{+}($LCH0005)

\begin{tabular}{|c|c|c|c|c|c|c|c|}
\hline \multicolumn{8}{|l|}{ HEADER } \\
\hline REMARK & Spart & an & 02 ez & orted & Molecule001 & & \\
\hline НЕТАТМ & 1 & $\mathrm{Na}$ & UNK & 0001 & 1.071 & -0.136 & 2.248 \\
\hline HETATM & 2 & $\mathrm{H}$ & UNK & 0001 & 2.957 & -1.213 & -0.332 \\
\hline HETATM & 3 & $\mathrm{C}$ & UNK & 0001 & 2.020 & -0.695 & -0.231 \\
\hline НЕТАТМ & 4 & $\mathrm{C}$ & UNK & 0001 & -0.368 & 0.693 & -0.087 \\
\hline НЕТАТМ & 5 & $\mathrm{C}$ & UNK & 0001 & 2.011 & 0.714 & -0.204 \\
\hline НЕTATM & 6 & $\mathrm{C}$ & UNK & 0001 & 0.838 & -1.413 & -0.177 \\
\hline HЕTATM & 7 & $\mathrm{C}$ & UNK & 0001 & -0.380 & -0.719 & -0.095 \\
\hline HETATM & 8 & $\mathrm{C}$ & UNK & 0001 & 0.825 & 1.423 & -0.127 \\
\hline HETATM & 9 & $\mathrm{H}$ & UNK & 0001 & 2.942 & 1.248 & -0.275 \\
\hline HETATM & 10 & $\mathrm{H}$ & UNK & 0001 & 0.851 & -2.487 & -0.228 \\
\hline НЕТАТМ & 11 & $\mathrm{H}$ & UNK & 0001 & 0.824 & 2.498 & -0.140 \\
\hline HETATM & 12 & $\mathrm{C}$ & UNK & 0001 & -1.763 & -1.120 & -0.058 \\
\hline
\end{tabular}




$\begin{array}{lrrrrrrr}\text { HETATM } & 13 & \text { H } & \text { UNK } & 0001 & -2.147 & -2.119 & -0.071 \\ \text { HETATM } & 14 & \text { C } & \text { UNK } & 0001 & -2.490 & 0.016 & -0.041 \\ \text { HETATM } & 15 & \text { H } & \text { UNK } & 0001 & -3.554 & 0.141 & -0.031 \\ \text { HETATM } & 16 & \text { N } & \text { UNK } & 0001 & -1.665 & 1.113 & -0.039 \\ \text { HETATM } & 17 & \text { H } & \text { UNK } & 0001 & -1.972 & 2.055 & -0.112 \\ \text { HETATM } & 18 & \text { Lig } & \text { UNK } & 0001 & 0.824 & 0.001 & -0.153 \\ \text { CONECT } & 1 & 18 & & & & & \\ \text { CONECT } & 2 & 3 & & & & \\ \text { CONECT } & 3 & 2 & 6 & 5 & & \\ \text { CONECT } & 4 & 7 & 8 & 16 & & \\ \text { CONECT } & 5 & 8 & 3 & 9 & & \\ \text { CONECT } & 6 & 3 & 7 & 10 & & & \\ \text { CONECT } & 7 & 6 & 4 & 12 & & & \\ \text { CONECT } & 8 & 4 & 5 & 11 & & & \\ \text { CONECT } & 9 & 5 & & & & & \\ \text { CONECT } & 10 & 6 & & & & & \\ \text { CONECT } & 11 & 8 & & & & & \\ \text { CONECT } & 12 & 13 & 7 & 14 & & & \\ \text { CONECT } & 13 & 12 & & & & & \\ \text { CONECT } & 14 & 15 & 12 & 16 & & & \\ \text { CONECT } & 15 & 14 & & & & & \\ \text { CONECT } & 16 & 17 & 14 & 4 & & & \\ \text { CONECT } & 17 & 16 & & & & & \\ \text { CONECT } & 18 & 1 & & & & & \\ \text { END } & & & & & & & \end{array}$

\section{4c. $\eta^{5}$-Indole $\mathrm{Na}^{+}(\mathrm{LCH0006})$}

\begin{tabular}{|c|c|c|c|c|c|c|c|}
\hline & & & & & & & \\
\hline REMARK & Spart & :an & $02 \mathrm{e}$ & ported & Molecule00 & & \\
\hline HETATM & 1 & $\mathrm{Na}$ & UNK & 0001 & -1.126 & -0.028 & -2.285 \\
\hline HETATM & 2 & $\mathrm{H}$ & UNK & 0001 & -2.020 & -2.111 & 0.272 \\
\hline HETATM & 3 & C & UNK & 0001 & -1.634 & -1.114 & 0.197 \\
\hline HETATM & 4 & C & UNK & 0001 & -2.365 & 0.024 & 0.220 \\
\hline НЕТАТМ & 5 & $\mathrm{H}$ & UNK & 0001 & -3.424 & 0.153 & 0.315 \\
\hline HETATM & 6 & $\mathrm{~N}$ & UNK & 0001 & -1.533 & 1.123 & 0.108 \\
\hline НЕTATM & 7 & $\mathrm{H}$ & UNK & 0001 & -1.812 & 2.044 & 0.365 \\
\hline HETATM & 8 & $\mathrm{C}$ & UNK & 0001 & -0.219 & 0.683 & 0.126 \\
\hline HETATM & 9 & $\mathrm{C}$ & UNK & 0001 & -0.239 & -0.718 & 0.139 \\
\hline НЕTATM & 10 & $\mathrm{C}$ & UNK & 0001 & 0.975 & -1.424 & 0.100 \\
\hline HETATM & 11 & $\mathrm{H}$ & UNK & 0001 & 0.984 & -2.499 & 0.123 \\
\hline HETATM & 12 & $\mathrm{C}$ & UNK & 0001 & 2.148 & -0.713 & 0.057 \\
\hline HETATM & 13 & $\mathrm{H}$ & UNK & 0001 & 3.088 & -1.233 & 0.040 \\
\hline HETATM & 14 & $\mathrm{C}$ & UNK & 0001 & 2.145 & 0.695 & 0.047 \\
\hline НЕTATM & 15 & $\mathrm{H}$ & UNK & 0001 & 3.083 & 1.220 & 0.024 \\
\hline НЕTATM & 16 & $\mathrm{C}$ & UNK & 0001 & 0.973 & 1.412 & 0.074 \\
\hline HETATM & 17 & $\mathrm{H}$ & UNK & 0001 & 0.976 & 2.487 & 0.078 \\
\hline
\end{tabular}




$\begin{array}{lrrrrrrr}\text { HETATM } & 18 & \text { Lig } & \text { UNK } & 0001 & -1.198 & -0.001 & 0.158 \\ \text { CONECT } & 1 & 18 & & & & \\ \text { CONECT } & 2 & 3 & & & & \\ \text { CONECT } & 3 & 2 & 9 & 4 & & \\ \text { CONECT } & 4 & 6 & 3 & 5 & & \\ \text { CONECT } & 5 & 4 & & & & \\ \text { CONECT } & 6 & 8 & 4 & 7 & & \\ \text { CONECT } & 7 & 6 & & & & \\ \text { CONECT } & 8 & 9 & 6 & 16 & & \\ \text { CONECT } & 9 & 3 & 8 & 10 & & \\ \text { CONECT } & 10 & 11 & 9 & 12 & & \\ \text { CONECT } & 11 & 10 & & & & \\ \text { CONECT } & 12 & 13 & 10 & 14 & & \\ \text { CONECT } & 13 & 12 & & & & \\ \text { CONECT } & 14 & 15 & 12 & 16 & & \\ \text { CONECT } & 15 & 14 & & & & \\ \text { CONECT } & 16 & 17 & 14 & 8 & & \\ \text { CONECT } & 17 & 16 & & & & \\ \text { CONECT } & 18 & 1 & & & & \\ \text { END } & & & & & & \end{array}$

15a. $N$-Methylindole (LCH0008)

\begin{tabular}{|c|c|c|c|c|c|c|c|}
\hline $\begin{array}{l}\text { HEADER } \\
\text { REMARK }\end{array}$ & Spart & & $02 \mathrm{ex}$ & ported & Molecule001 & & \\
\hline & 1 & $\mathrm{C}$ & UNK & 0001 & $\begin{array}{c}\text { Molecule } 001 \\
0.058\end{array}$ & -0.201 & 0.002 \\
\hline HETATM & 2 & $\mathrm{C}$ & UNK & 0001 & -2.692 & -0.137 & -0.001 \\
\hline HETATM & 3 & $\mathrm{C}$ & UNK & 0001 & -0.608 & 1.033 & 0.000 \\
\hline НЕTATM & 4 & $\mathrm{C}$ & UNK & 0001 & -0.634 & -1.413 & 0.002 \\
\hline HETATM & 5 & $\mathrm{C}$ & UNK & 0001 & -2.008 & -1.362 & 0.001 \\
\hline HETATM & 6 & $\mathrm{C}$ & UNK & 0001 & -2.006 & 1.054 & -0.001 \\
\hline HETATM & 7 & $\mathrm{H}$ & UNK & 0001 & -0.117 & -2.356 & 0.002 \\
\hline HETATM & 8 & $\mathrm{H}$ & UNK & 0001 & -2.571 & -2.279 & 0.000 \\
\hline НЕTATM & 9 & $\mathrm{H}$ & UNK & 0001 & -2.536 & 1.991 & -0.002 \\
\hline HETATM & 10 & $\mathrm{H}$ & UNK & 0001 & -3.768 & -0.136 & -0.001 \\
\hline HETATM & 11 & $\mathrm{C}$ & UNK & 0001 & 0.412 & 2.049 & -0.000 \\
\hline HETATM & 12 & $\mathrm{C}$ & UNK & 0001 & 1.595 & 1.403 & 0.002 \\
\hline HETATM & 13 & $\mathrm{H}$ & UNK & 0001 & 2.588 & 1.805 & 0.002 \\
\hline HETATM & 14 & $\mathrm{~N}$ & UNK & 0001 & 1.408 & 0.042 & 0.004 \\
\hline HЕTATM & 15 & $\mathrm{H}$ & UNK & 0001 & 0.271 & 3.110 & -0.000 \\
\hline HETATM & 16 & $\mathrm{C}$ & UNK & 0001 & 2.443 & -0.959 & -0.002 \\
\hline HETATM & 17 & $\mathrm{H}$ & UNK & 0001 & 2.383 & -1.579 & -0.890 \\
\hline HETATM & 18 & $\mathrm{H}$ & UNK & 0001 & 2.374 & -1.596 & 0.874 \\
\hline HETATM & 19 & $\mathrm{H}$ & UNK & 0001 & 3.407 & -0.469 & 0.008 \\
\hline CONECT & 1 & & 3 & 14 & & & \\
\hline CONECT & 2 & & 5 & 10 & & & \\
\hline CONECT & 3 & & 6 & 11 & & & \\
\hline
\end{tabular}




$\begin{array}{lrrrrr}\text { CONECT } & 4 & 1 & 5 & 7 & \\ \text { CONECT } & 5 & 4 & 2 & 8 & \\ \text { CONECT } & 6 & 2 & 3 & 9 & \\ \text { CONECT } & 7 & 4 & & & \\ \text { CONECT } & 8 & 5 & & & \\ \text { CONECT } & 9 & 6 & & & \\ \text { CONECT } & 10 & 2 & & & \\ \text { CONECT } & 11 & 3 & 12 & 15 & \\ \text { CONECT } & 12 & 13 & 11 & 14 & \\ \text { CONECT } & 13 & 12 & & & \\ \text { CONECT } & 14 & 12 & 1 & 16 & \\ \text { CONECT } & 15 & 11 & & & \\ \text { CONECT } & 16 & 17 & 18 & 19 & 14 \\ \text { CONECT } & 17 & 16 & & & \\ \text { CONECT } & 18 & 16 & & & \\ \text { CONECT } & 19 & 16 & & & \\ \text { END } & & & & & \end{array}$

\section{5b. $\eta^{6}-(N$-Methylindole $) \cdot \mathrm{Na}^{+}(\mathrm{LCH0009})$}

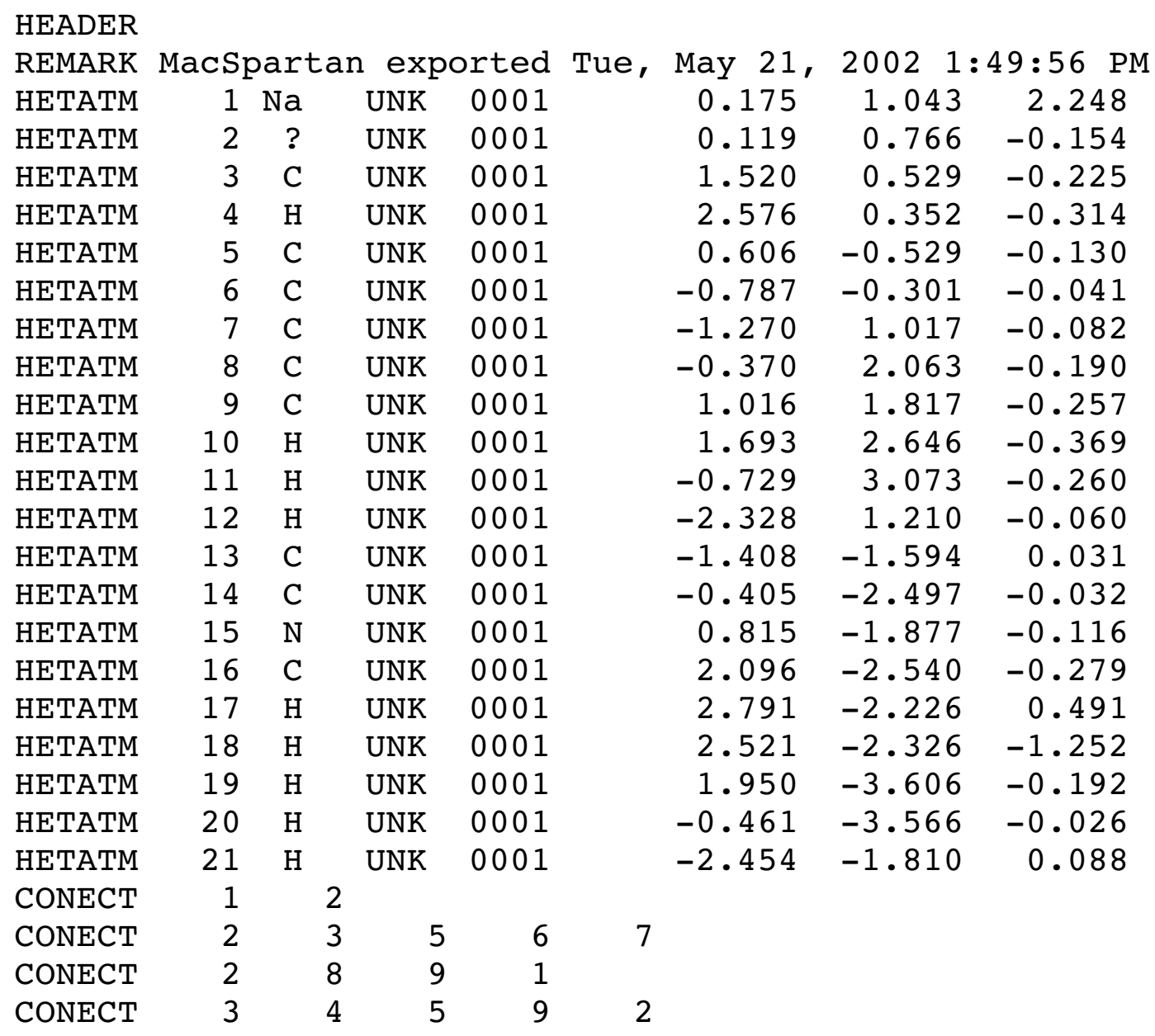




$\begin{array}{lrrrrr}\text { CONECT } & 4 & 3 & & & \\ \text { CONECT } & 5 & 3 & 6 & 15 & 2 \\ \text { CONECT } & 6 & 5 & 7 & 13 & 2 \\ \text { CONECT } & 7 & 6 & 8 & 12 & 2 \\ \text { CONECT } & 8 & 7 & 9 & 11 & 2 \\ \text { CONECT } & 9 & 8 & 3 & 10 & 2 \\ \text { CONECT } & 10 & 9 & & & \\ \text { CONECT } & 11 & 8 & & & \\ \text { CONECT } & 12 & 7 & & & \\ \text { CONECT } & 13 & 6 & 14 & 21 & \\ \text { CONECT } & 14 & 13 & 15 & 20 & \\ \text { CONECT } & 15 & 14 & 5 & 16 & \\ \text { CONECT } & 16 & 15 & 17 & 18 & 19 \\ \text { CONECT } & 17 & 16 & & & \\ \text { CONECT } & 18 & 16 & & & \\ \text { CONECT } & 19 & 16 & & & \\ \text { CONECT } & 20 & 14 & & & \\ \text { CONECT } & 21 & 13 & & & \\ \text { END } & & & & & \end{array}$

\section{5c. $\eta^{5}-(N$-Methylindole $) \cdot \mathrm{Na}^{+}($LCH0010 $)$}

\begin{tabular}{|c|c|c|c|c|c|c|c|}
\hline HEADER & & & & & & & \\
\hline REMARK & Spart & an & $02 \mathrm{ex}$ & ported & Molecule001 & & \\
\hline НЕТАТМ & 1 & $\mathrm{Na}$ & UNK & 0001 & 0.033 & 1.064 & 2.325 \\
\hline HETATM & 2 & $\mathrm{~N}$ & UNK & 0001 & 1.411 & -0.012 & 0.000 \\
\hline HETATM & 3 & $\mathrm{C}$ & UNK & 0001 & 1.606 & 1.340 & -0.129 \\
\hline HETATM & 4 & $\mathrm{H}$ & UNK & 0001 & 2.599 & 1.739 & -0.173 \\
\hline HETATM & 5 & C & UNK & 0001 & 0.418 & 1.993 & -0.204 \\
\hline НЕТАТМ & 6 & $\mathrm{H}$ & UNK & 0001 & 0.285 & 3.047 & -0.342 \\
\hline HETATM & 7 & $\mathrm{C}$ & UNK & 0001 & -0.610 & 0.975 & -0.170 \\
\hline HETATM & 8 & $\mathrm{C}$ & UNK & 0001 & 0.054 & -0.257 & -0.057 \\
\hline HETATM & 9 & $\mathrm{C}$ & UNK & 0001 & -0.645 & -1.469 & 0.020 \\
\hline НЕTATM & 10 & $\mathrm{H}$ & UNK & 0001 & -0.135 & -2.413 & 0.085 \\
\hline НЕТАТМ & 11 & $\mathrm{C}$ & UNK & 0001 & -2.017 & -1.410 & -0.018 \\
\hline HETATM & 12 & $\mathrm{H}$ & UNK & 0001 & -2.584 & -2.322 & 0.026 \\
\hline НЕТАТМ & 13 & C & UNK & 0001 & -2.700 & -0.184 & -0.126 \\
\hline НЕTATM & 14 & $\mathrm{H}$ & UNK & 0001 & -3.774 & -0.185 & -0.163 \\
\hline НЕTАTM & 15 & C & UNK & 0001 & -2.016 & 1.004 & -0.202 \\
\hline НЕТАТМ & 16 & $\mathrm{H}$ & UNK & 0001 & -2.542 & 1.936 & -0.306 \\
\hline НЕTATM & 17 & C & UNK & 0001 & 2.452 & -1.022 & -0.122 \\
\hline НЕTАТМ & 18 & $\mathrm{H}$ & UNK & 0001 & 3.412 & -0.558 & 0.049 \\
\hline НЕТАТМ & 19 & $\mathrm{H}$ & UNK & 0001 & 2.444 & -1.465 & -1.110 \\
\hline НЕTATM & 20 & $\mathrm{H}$ & UNK & 0001 & 2.308 & -1.800 & 0.617 \\
\hline НЕТАТМ & 21 & Lig & UNK & 0001 & 0.576 & 0.808 & -0.112 \\
\hline CONECT & 1 & 21 & & & & & \\
\hline CONECT & 2 & 8 & 3 & 17 & & & \\
\hline
\end{tabular}




$\begin{array}{lrrrr}\text { CONECT } & 3 & 5 & 2 & 4 \\ \text { CONECT } & 4 & 3 & & \\ \text { CONECT } & 5 & 7 & 3 & 6 \\ \text { CONECT } & 6 & 5 & & \\ \text { CONECT } & 7 & 8 & 5 & 15 \\ \text { CONECT } & 8 & 2 & 7 & 9 \\ \text { CONECT } & 9 & 10 & 8 & 11 \\ \text { CONECT } & 10 & 9 & & \\ \text { CONECT } & 11 & 12 & 9 & 13 \\ \text { CONECT } & 12 & 11 & & \\ \text { CONECT } & 13 & 14 & 11 & 15 \\ \text { CONECT } & 14 & 13 & & \\ \text { CONECT } & 15 & 16 & 13 & 7 \\ \text { CONECT } & 16 & 15 & & \\ \text { CONECT } & 17 & 18 & 19 & 20 \\ \text { CONECT } & 18 & 17 & & \\ \text { CONECT } & 19 & 17 & & \\ \text { CONECT } & 20 & 17 & & \\ \text { CONECT } & 21 & 1 & & \\ \text { END } & & & & \end{array}$

16a. 2-Methylindole (LCH0012)

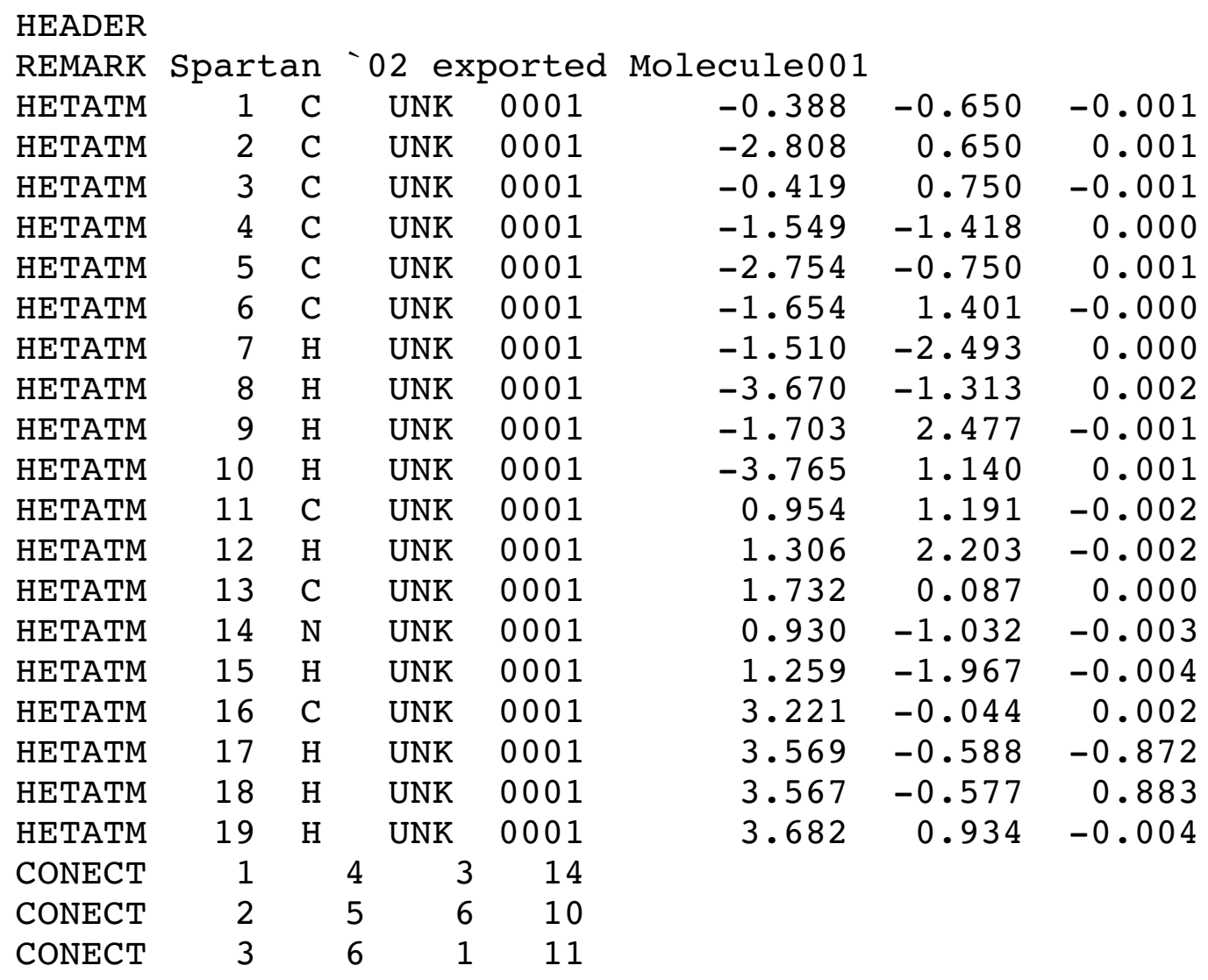




$\begin{array}{lrrrrr}\text { CONECT } & 4 & 1 & 5 & 7 & \\ \text { CONECT } & 5 & 4 & 2 & 8 & \\ \text { CONECT } & 6 & 2 & 3 & 9 & \\ \text { CONECT } & 7 & 4 & & & \\ \text { CONECT } & 8 & 5 & & & \\ \text { CONECT } & 9 & 6 & & & \\ \text { CONECT } & 10 & 2 & & & \\ \text { CONECT } & 11 & 12 & 3 & 13 & \\ \text { CONECT } & 12 & 11 & & & \\ \text { CONECT } & 13 & 11 & 14 & 16 & \\ \text { CONECT } & 14 & 15 & 13 & 1 & \\ \text { CONECT } & 15 & 14 & & & \\ \text { CONECT } & 16 & 17 & 18 & 19 & 13 \\ \text { CONECT } & 17 & 16 & & & \\ \text { CONECT } & 18 & 16 & & & \\ \text { CONECT } & 19 & 16 & & & \\ \text { END } & & & & & \end{array}$

16b. $\eta^{6}-(2-M e t h y l i n d o l e) \cdot \mathrm{Na}^{+}(\mathrm{LCHO013})$

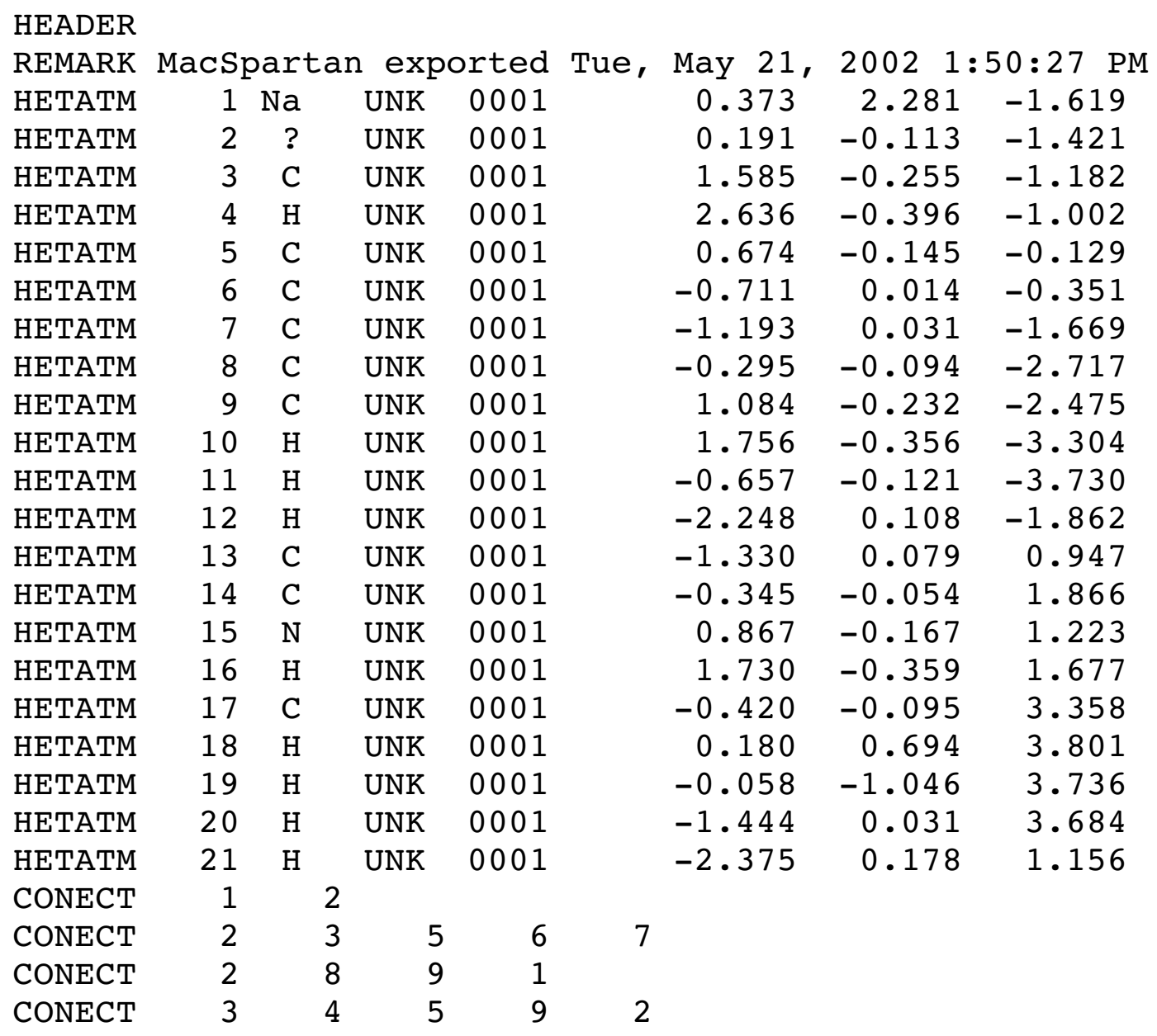




$\begin{array}{lrrrrr}\text { CONECT } & 4 & 3 & & & \\ \text { CONECT } & 5 & 3 & 6 & 15 & 2 \\ \text { CONECT } & 6 & 5 & 7 & 13 & 2 \\ \text { CONECT } & 7 & 6 & 8 & 12 & 2 \\ \text { CONECT } & 8 & 7 & 9 & 11 & 2 \\ \text { CONECT } & 9 & 8 & 3 & 10 & 2 \\ \text { CONECT } & 10 & 9 & & & \\ \text { CONECT } & 11 & 8 & & & \\ \text { CONECT } & 12 & 7 & & & \\ \text { CONECT } & 13 & 6 & 14 & 21 & \\ \text { CONECT } & 14 & 13 & 15 & 17 & \\ \text { CONECT } & 15 & 14 & 5 & 16 & \\ \text { CONECT } & 16 & 15 & & & \\ \text { CONECT } & 17 & 14 & 18 & 19 & 20 \\ \text { CONECT } & 18 & 17 & & & \\ \text { CONECT } & 19 & 17 & & & \\ \text { CONECT } & 20 & 17 & & & \\ \text { CONECT } & 21 & 13 & & & \\ \text { END } & & & & & \end{array}$

16c. $\eta^{5}$-(2-Methylindole) $\bullet \mathrm{Na}^{+}(\mathrm{LCH0014})$

\begin{tabular}{|c|c|c|c|c|c|c|c|}
\hline HEADER & & & & & & & \\
\hline REMARK & Spart & Ean & $02 \mathrm{exp}$ & ported & Molecule001 & & \\
\hline НETATM & 1 & $\mathrm{Na}$ & UNK & 0001 & 0.166 & 0.190 & -2.364 \\
\hline НЕТАТМ & 2 & C & UNK & 0001 & -0.402 & -0.660 & 0.083 \\
\hline НЕТАТМ & 3 & $\mathrm{C}$ & UNK & 0001 & -0.432 & 0.740 & 0.113 \\
\hline НЕТАТМ & 4 & $\mathrm{H}$ & UNK & 0001 & 1.252 & -1.964 & 0.200 \\
\hline НЕТАТМ & 5 & $\mathrm{C}$ & UNK & 0001 & 0.950 & 1.184 & 0.089 \\
\hline НЕТАТМ & 6 & $\mathrm{H}$ & UNK & 0001 & 1.301 & 2.194 & 0.160 \\
\hline НЕТАТМ & 7 & $\mathrm{C}$ & UNK & 0001 & 1.734 & 0.076 & 0.069 \\
\hline НЕТАТМ & 8 & $\mathrm{~N}$ & UNK & 0001 & 0.927 & -1.046 & -0.009 \\
\hline НЕТАТМ & 9 & $\mathrm{C}$ & UNK & 0001 & -1.565 & -1.433 & 0.073 \\
\hline HETATM & 10 & $\mathrm{H}$ & UNK & 0001 & -1.527 & -2.508 & 0.062 \\
\hline HETATM & 11 & $\mathrm{C}$ & UNK & 0001 & -2.766 & -0.762 & 0.108 \\
\hline НЕТАТМ & 12 & $\mathrm{H}$ & UNK & 0001 & -3.683 & -1.322 & 0.120 \\
\hline НETATM & 13 & C & UNK & 0001 & -2.820 & 0.642 & 0.136 \\
\hline НЕТАТМ & 14 & $\mathrm{H}$ & UNK & 0001 & -3.778 & 1.128 & 0.168 \\
\hline HETATM & 15 & $\mathrm{C}$ & UNK & 0001 & -1.672 & 1.398 & 0.135 \\
\hline НЕТАТМ & 16 & $\mathrm{H}$ & UNK & 0001 & -1.721 & 2.472 & 0.172 \\
\hline НЕТАТМ & 17 & C & UNK & 0001 & 3.223 & -0.057 & 0.150 \\
\hline HETATM & 18 & $\mathrm{H}$ & UNK & 0001 & 3.612 & -0.696 & -0.636 \\
\hline НЕТАТМ & 19 & $\mathrm{H}$ & UNK & 0001 & 3.510 & -0.492 & 1.103 \\
\hline НЕTATM & 20 & $\mathrm{H}$ & UNK & 0001 & 3.692 & 0.914 & 0.070 \\
\hline НЕTATM & 21 & Lig & UNK & 0001 & 0.555 & 0.059 & 0.069 \\
\hline CONECT & 1 & 21 & & & & & \\
\hline CONECT & 2 & 8 & 3 & 9 & & & \\
\hline
\end{tabular}




$\begin{array}{lrrrr}\text { CONECT } & 3 & 5 & 2 & 15 \\ \text { CONECT } & 4 & 8 & & \\ \text { CONECT } & 5 & 7 & 3 & 6 \\ \text { CONECT } & 6 & 5 & & \\ \text { CONECT } & 7 & 8 & 5 & 17 \\ \text { CONECT } & 8 & 2 & 7 & 4 \\ \text { CONECT } & 9 & 10 & 2 & 11 \\ \text { CONECT } & 10 & 9 & & \\ \text { CONECT } & 11 & 12 & 9 & 13 \\ \text { CONECT } & 12 & 11 & & \\ \text { CONECT } & 13 & 14 & 11 & 15 \\ \text { CONECT } & 14 & 13 & & \\ \text { CONECT } & 15 & 16 & 13 & 3 \\ \text { CONECT } & 16 & 15 & & \\ \text { CONECT } & 17 & 18 & 19 & 20 \\ \text { CONECT } & 18 & 17 & & \\ \text { CONECT } & 19 & 17 & & \\ \text { CONECT } & 20 & 17 & & \\ \text { CONECT } & 21 & 1 & & \\ \text { END } & & & & \end{array}$

17a. 3-Methylindole (LCH0016)

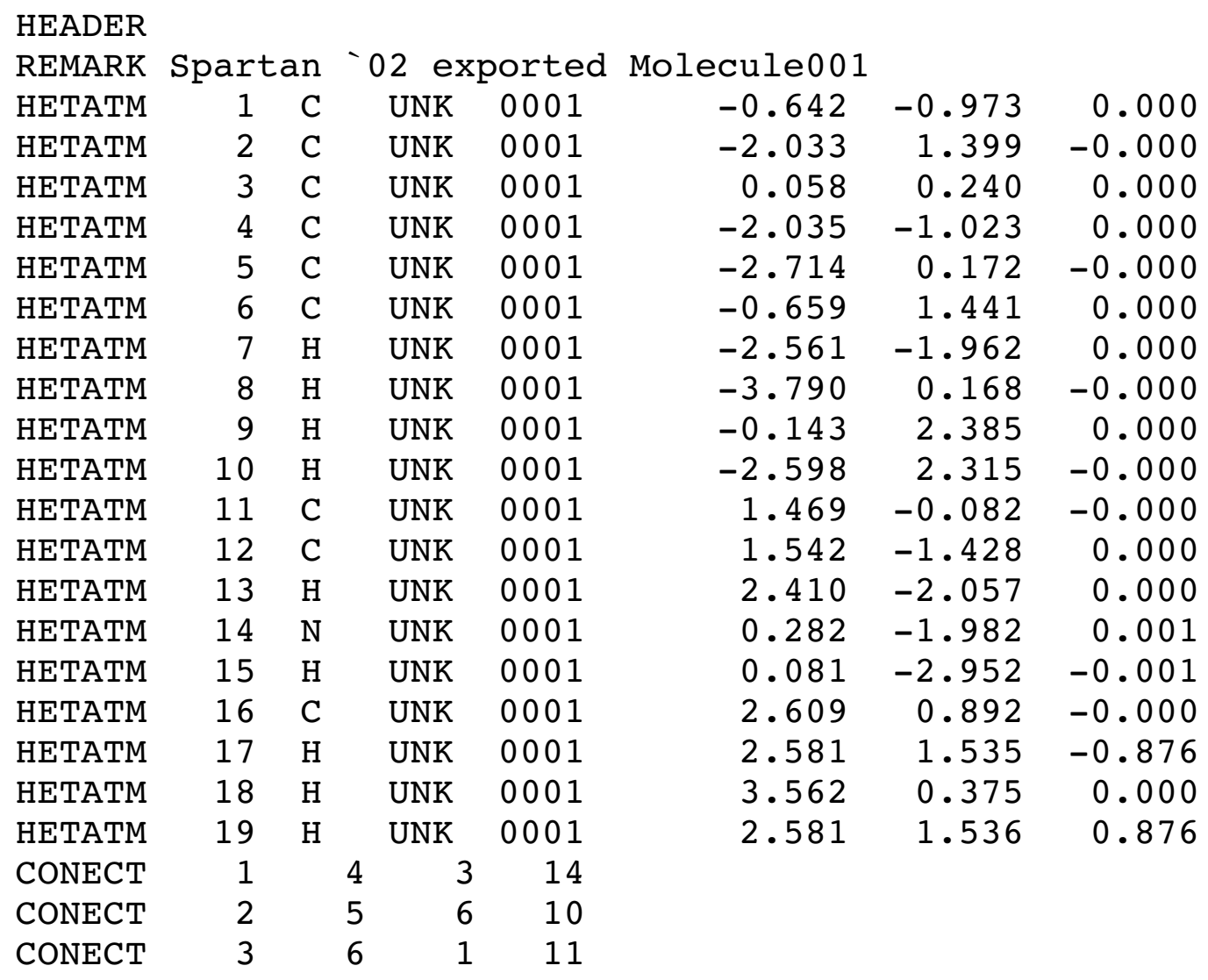




$\begin{array}{lrrrrr}\text { CONECT } & 4 & 1 & 5 & 7 & \\ \text { CONECT } & 5 & 4 & 2 & 8 & \\ \text { CONECT } & 6 & 2 & 3 & 9 & \\ \text { CONECT } & 7 & 4 & & & \\ \text { CONECT } & 8 & 5 & & & \\ \text { CONECT } & 9 & 6 & & & \\ \text { CONECT } & 10 & 2 & & & \\ \text { CONECT } & 11 & 3 & 12 & 16 & \\ \text { CONECT } & 12 & 13 & 11 & 14 & \\ \text { CONECT } & 13 & 12 & & & \\ \text { CONECT } & 14 & 15 & 12 & 1 & \\ \text { CONECT } & 15 & 14 & & & \\ \text { CONECT } & 16 & 17 & 18 & 19 & 11 \\ \text { CONECT } & 17 & 16 & & & \\ \text { CONECT } & 18 & 16 & & & \\ \text { CONECT } & 19 & 16 & & & \\ \text { END } & & & & & \end{array}$

17b. $\eta^{6}-(3-M e t h y l i n d o l e) \cdot \mathrm{Na}^{+}(\mathrm{LCHO017})$

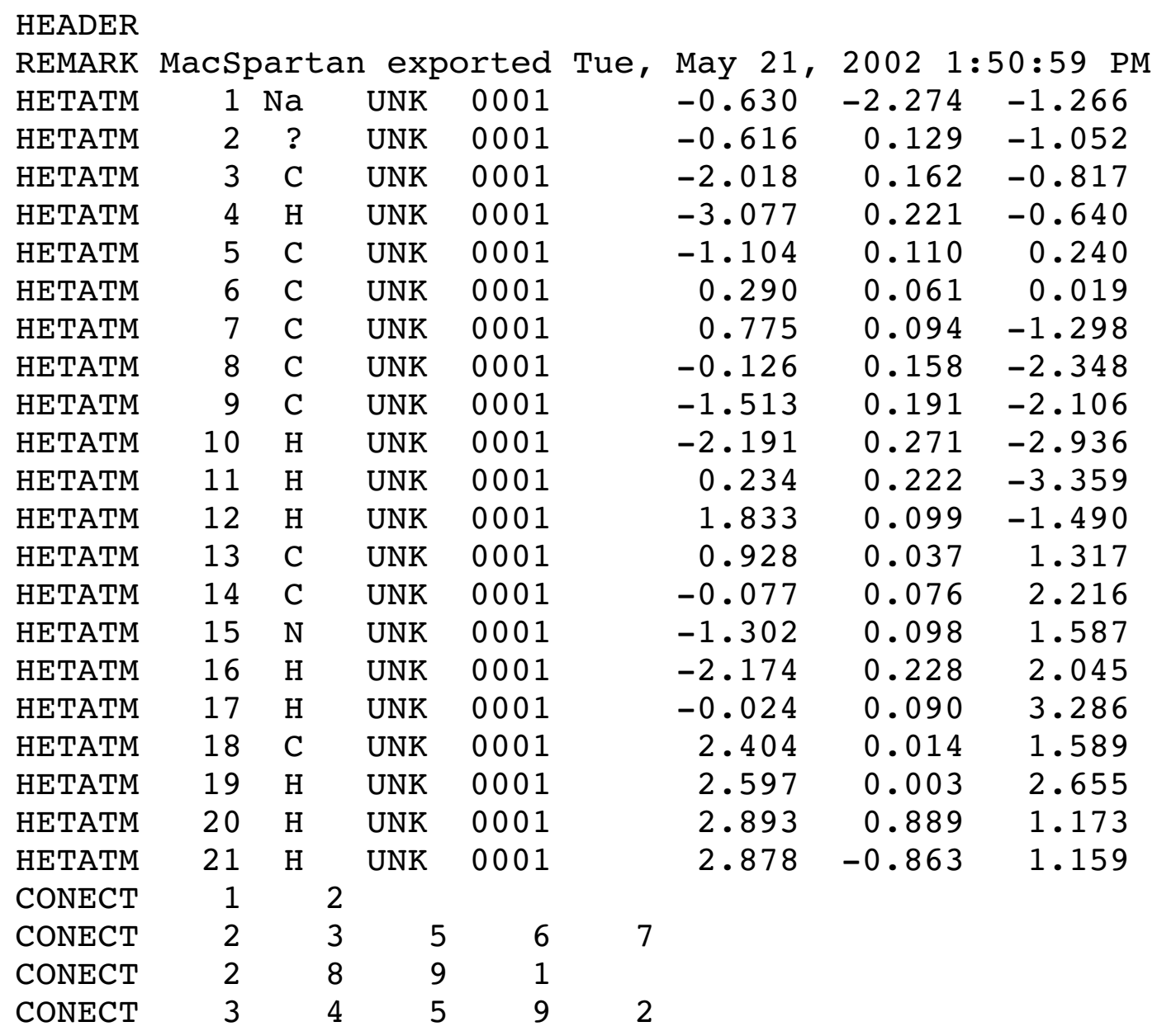




$\begin{array}{lrrrrr}\text { CONECT } & 4 & 3 & & & \\ \text { CONECT } & 5 & 3 & 6 & 15 & 2 \\ \text { CONECT } & 6 & 5 & 7 & 13 & 2 \\ \text { CONECT } & 7 & 6 & 8 & 12 & 2 \\ \text { CONECT } & 8 & 7 & 9 & 11 & 2 \\ \text { CONECT } & 9 & 8 & 3 & 10 & 2 \\ \text { CONECT } & 10 & 9 & & & \\ \text { CONECT } & 11 & 8 & & & \\ \text { CONECT } & 12 & 7 & & & \\ \text { CONECT } & 13 & 6 & 14 & 18 & \\ \text { CONECT } & 14 & 13 & 15 & 17 & \\ \text { CONECT } & 15 & 14 & 5 & 16 & \\ \text { CONECT } & 16 & 15 & & & \\ \text { CONECT } & 17 & 14 & & & \\ \text { CONECT } & 18 & 13 & 19 & 20 & 21 \\ \text { CONECT } & 19 & 18 & & & \\ \text { CONECT } & 20 & 18 & & & \\ \text { CONECT } & 21 & 18 & & & \\ \text { END } & & & & & \end{array}$

17c. $\eta^{5}$-(3-Methylindole) $\cdot \mathrm{Na}^{+}(\mathrm{LCH0018})$

\begin{tabular}{|c|c|c|c|c|c|c|c|}
\hline HEADER & & & & & & & \\
\hline REMARK & Spart & Ean & $02 \mathrm{exp}$ & orted & Molecule001 & & \\
\hline НЕTATM & 1 & $\mathrm{Na}$ & UNK & 0001 & 0.330 & -1.099 & -2.296 \\
\hline НЕTATM & 2 & $\mathrm{C}$ & UNK & 0001 & -0.656 & -0.923 & 0.131 \\
\hline HETATM & 3 & $\mathrm{C}$ & UNK & 0001 & 0.034 & 0.291 & 0.058 \\
\hline НЕТАТМ & 4 & $\mathrm{H}$ & UNK & 0001 & 0.106 & -2.844 & 0.501 \\
\hline HETATM & 5 & $\mathrm{C}$ & UNK & 0001 & 1.460 & -0.017 & 0.084 \\
\hline НЕTATM & 6 & $\mathrm{C}$ & UNK & 0001 & 1.551 & -1.363 & 0.155 \\
\hline НЕТАТМ & 7 & $\mathrm{~N}$ & UNK & 0001 & 0.283 & -1.948 & 0.100 \\
\hline HETATM & 8 & $\mathrm{C}$ & UNK & 0001 & -2.050 & -0.989 & 0.144 \\
\hline HETATM & 9 & $\mathrm{H}$ & UNK & 0001 & -2.569 & -1.929 & 0.214 \\
\hline HETATM & 10 & $\mathrm{C}$ & UNK & 0001 & -2.735 & 0.203 & 0.096 \\
\hline HETATM & 11 & $\mathrm{H}$ & UNK & 0001 & -3.810 & 0.194 & 0.122 \\
\hline НЕTATM & 12 & $\mathrm{C}$ & UNK & 0001 & -2.063 & 1.434 & 0.018 \\
\hline НЕTАТМ & 13 & $\mathrm{H}$ & UNK & 0001 & -2.636 & 2.342 & -0.015 \\
\hline НЕTATM & 14 & $\mathrm{C}$ & UNK & 0001 & -0.690 & 1.492 & -0.004 \\
\hline HETATM & 15 & $\mathrm{H}$ & UNK & 0001 & -0.182 & 2.438 & -0.050 \\
\hline НETATM & 16 & $\mathrm{H}$ & UNK & 0001 & 2.423 & -1.979 & 0.245 \\
\hline НЕТАТМ & 17 & $\mathrm{C}$ & UNK & 0001 & 2.584 & 0.979 & 0.114 \\
\hline НЕTATM & 18 & $\mathrm{H}$ & UNK & 0001 & 2.546 & 1.656 & -0.734 \\
\hline НЕTATM & 19 & $\mathrm{H}$ & UNK & 0001 & 3.546 & 0.480 & 0.103 \\
\hline HETATM & 20 & $\mathrm{H}$ & UNK & 0001 & 2.529 & 1.582 & 1.014 \\
\hline НЕTАТМ & 21 & Lig & UNK & 0001 & 0.535 & -0.792 & 0.106 \\
\hline CONECT & 1 & 21 & & & & & \\
\hline CONECT & 2 & 7 & 3 & 8 & & & \\
\hline
\end{tabular}




$\begin{array}{lrrrr}\text { CONECT } & 3 & 5 & 2 & 14 \\ \text { CONECT } & 4 & 7 & & \\ \text { CONECT } & 5 & 6 & 3 & 17 \\ \text { CONECT } & 6 & 7 & 5 & 16 \\ \text { CONECT } & 7 & 2 & 6 & 4 \\ \text { CONECT } & 8 & 9 & 2 & 10 \\ \text { CONECT } & 9 & 8 & & \\ \text { CONECT } & 10 & 11 & 8 & 12 \\ \text { CONECT } & 11 & 10 & & \\ \text { CONECT } & 12 & 13 & 10 & 14 \\ \text { CONECT } & 13 & 12 & & \\ \text { CONECT } & 14 & 15 & 12 & 3 \\ \text { CONECT } & 15 & 14 & & \\ \text { CONECT } & 16 & 6 & & \\ \text { CONECT } & 17 & 18 & 19 & 20 \\ \text { CONECT } & 18 & 17 & & \\ \text { CONECT } & 19 & 17 & & \\ \text { CONECT } & 20 & 17 & & \\ \text { CONECT } & 21 & 1 & & \\ \text { END } & & & & \end{array}$

18a. 4-Methylindole (LCH0020)

\begin{tabular}{|c|c|c|c|c|c|c|c|}
\hline HEADER & & & & & & & \\
\hline REMARK & Spar & & $02 \mathrm{ex}$ & orted & Molecule001 & & \\
\hline НЕТАТМ & 1 & $\mathrm{C}$ & UNK & 0001 & 1.128 & 0.649 & -0.000 \\
\hline НЕTATM & 2 & $\mathrm{C}$ & UNK & 0001 & -1.567 & 1.137 & 0.000 \\
\hline НЕТАТМ & 3 & C & UNK & 0001 & 0.229 & -0.422 & 0.000 \\
\hline НЕTATM & 4 & $\mathrm{C}$ & UNK & 0001 & 0.705 & 1.979 & -0.000 \\
\hline HETATM & 5 & C & UNK & 0001 & -0.649 & 2.202 & -0.000 \\
\hline НЕТАТМ & 6 & $\mathrm{C}$ & UNK & 0001 & -1.155 & -0.176 & 0.000 \\
\hline НЕТАТМ & 7 & $\mathrm{H}$ & UNK & 0001 & 1.408 & 2.794 & -0.000 \\
\hline НЕTАТМ & 8 & $\mathrm{H}$ & UNK & 0001 & -1.020 & 3.212 & -0.000 \\
\hline HETATM & 9 & $\mathrm{H}$ & UNK & 0001 & -2.619 & 1.362 & 0.000 \\
\hline HETATM & 10 & C & UNK & 0001 & 1.023 & -1.627 & -0.000 \\
\hline НЕТАТМ & 11 & C & UNK & 0001 & 2.315 & -1.241 & 0.000 \\
\hline НЕTATM & 12 & $\mathrm{H}$ & UNK & 0001 & 3.205 & -1.837 & 0.000 \\
\hline НЕТАТМ & 13 & $\mathrm{~N}$ & UNK & 0001 & 2.395 & 0.128 & 0.000 \\
\hline НЕТАТМ & 14 & $\mathrm{H}$ & UNK & 0001 & 3.235 & 0.654 & 0.001 \\
\hline HETATM & 15 & $\mathrm{H}$ & UNK & 0001 & 0.671 & -2.638 & -0.000 \\
\hline НETATM & 16 & $\mathrm{C}$ & UNK & 0001 & -2.137 & -1.320 & 0.000 \\
\hline HETATM & 17 & $\mathrm{H}$ & UNK & 0001 & -3.159 & -0.958 & 0.001 \\
\hline HETATM & 18 & $\mathrm{H}$ & UNK & 0001 & -2.004 & -1.950 & 0.875 \\
\hline НETATM & 19 & $\mathrm{H}$ & UNK & 0001 & -2.005 & -1.949 & -0.876 \\
\hline CONECT & 1 & & 3 & 13 & & & \\
\hline CONECT & 2 & & 6 & 9 & & & \\
\hline NECT & 3 & & 1 & 10 & & & \\
\hline
\end{tabular}




$\begin{array}{lrrrr}\text { CONECT } & 4 & 1 & 5 & 7 \\ \text { CONECT } & 5 & 4 & 2 & 8 \\ \text { CONECT } & 6 & 2 & 3 & 16 \\ \text { CONECT } & 7 & 4 & & \\ \text { CONECT } & 8 & 5 & & \\ \text { CONECT } & 9 & 2 & & \\ \text { CONECT } & 10 & 3 & 11 & 15 \\ \text { CONECT } & 11 & 12 & 10 & 13 \\ \text { CONECT } & 12 & 11 & & \\ \text { CONECT } & 13 & 14 & 11 & 1 \\ \text { CONECT } & 14 & 13 & & \\ \text { CONECT } & 15 & 10 & & \\ \text { CONECT } & 16 & 17 & 18 & 19 \\ \text { CONECT } & 17 & 16 & & \\ \text { CONECT } & 18 & 16 & & \\ \text { CONECT } & 19 & 16 & & \\ \text { END } & & & & \end{array}$

18b. $\eta^{6}-(4-M e t h y l i n d o l e) \cdot \mathrm{Na}^{+}($LCHO021)

\begin{tabular}{|c|c|c|c|c|c|c|c|c|}
\hline HEADER & & & & & & & & \\
\hline REMARK & $\operatorname{MacSp}$ & artan & exp & orted & Tue, & May 21 , & 20021 & $51: 29 \quad \mathrm{PM}$ \\
\hline HETATM & 1 & $\mathrm{Na}$ & UNK & 0001 & & 0.679 & 2.282 & -0.706 \\
\hline HETATM & 2 & ? & UNK & 0001 & & 0.594 & -0.111 & -0.578 \\
\hline НЕТАТМ & 3 & $\mathrm{C}$ & UNK & 0001 & & 2.003 & -0.155 & -0.335 \\
\hline НЕTATM & 4 & $\mathrm{H}$ & UNK & 0001 & & 3.060 & -0.227 & -0.148 \\
\hline HETATM & 5 & C & UNK & 0001 & & 1.074 & -0.096 & 0.713 \\
\hline НЕTATM & 6 & $\mathrm{C}$ & UNK & 0001 & & -0.314 & -0.034 & 0.482 \\
\hline HETATM & 7 & $\mathrm{C}$ & UNK & 0001 & & -0.815 & -0.073 & -0.838 \\
\hline HETATM & 8 & $\mathrm{C}$ & UNK & 0001 & & 0.112 & -0.130 & -1.868 \\
\hline HETATM & 9 & C & UNK & 0001 & & 1.501 & -0.176 & -1.620 \\
\hline HETATM & 10 & $\mathrm{H}$ & UNK & 0001 & & 2.177 & -0.256 & -2.452 \\
\hline HETATM & 11 & $\mathrm{H}$ & UNK & 0001 & & -0.232 & -0.188 & -2.886 \\
\hline HETATM & 12 & $\mathrm{C}$ & UNK & 0001 & & -2.301 & -0.106 & -1.100 \\
\hline НЕTATM & 13 & $\mathrm{H}$ & UNK & 0001 & & -2.516 & -0.017 & -2.157 \\
\hline HETATM & 14 & $\mathrm{H}$ & UNK & 0001 & & -2.819 & 0.691 & -0.576 \\
\hline HETATM & 15 & $\mathrm{H}$ & UNK & 0001 & & -2.717 & -1.046 & -0.751 \\
\hline HETATM & 16 & $\mathrm{C}$ & UNK & 0001 & & -0.945 & 0.002 & 1.778 \\
\hline HETATM & 17 & $\mathrm{C}$ & UNK & 0001 & & 0.048 & -0.046 & 2.691 \\
\hline HETATM & 18 & $\mathrm{~N}$ & UNK & 0001 & & 1.267 & -0.085 & 2.064 \\
\hline HETATM & 19 & $\mathrm{H}$ & UNK & 0001 & & 2.139 & -0.218 & 2.523 \\
\hline HETATM & 20 & $\mathrm{H}$ & UNK & 0001 & & -0.011 & -0.056 & 3.760 \\
\hline НЕТАТМ & 21 & $\mathrm{H}$ & UNK & 0001 & & -1.993 & 0.034 & 1.990 \\
\hline CONECT & 1 & 2 & & & & & & \\
\hline CONECT & 2 & 3 & 5 & 6 & 7 & & & \\
\hline CONECT & 2 & 8 & 9 & 1 & & & & \\
\hline CONECT & 3 & 4 & 5 & 9 & 2 & & & \\
\hline
\end{tabular}




$\begin{array}{lrrrrr}\text { CONECT } & 4 & 3 & & & \\ \text { CONECT } & 5 & 3 & 6 & 18 & 2 \\ \text { CONECT } & 6 & 5 & 7 & 16 & 2 \\ \text { CONECT } & 7 & 6 & 8 & 12 & 2 \\ \text { CONECT } & 8 & 7 & 9 & 11 & 2 \\ \text { CONECT } & 9 & 8 & 3 & 10 & 2 \\ \text { CONECT } & 10 & 9 & & & \\ \text { CONECT } & 11 & 8 & & & \\ \text { CONECT } & 12 & 7 & 13 & 14 & 15 \\ \text { CONECT } & 13 & 12 & & & \\ \text { CONECT } & 14 & 12 & & & \\ \text { CONECT } & 15 & 12 & & & \\ \text { CONECT } & 16 & 6 & 17 & 21 & \\ \text { CONECT } & 17 & 16 & 18 & 20 & \\ \text { CONECT } & 18 & 17 & 5 & 19 & \\ \text { CONECT } & 19 & 18 & & & \\ \text { CONECT } & 20 & 17 & & & \\ \text { CONECT } & 21 & 16 & & & \\ \text { END } & & & & & \end{array}$

18c. $\eta^{5}$-(4-Methylindole) $\bullet \mathrm{Na}^{+}(\mathrm{LCH0022})$

\begin{tabular}{|c|c|c|c|c|c|c|c|}
\hline HEADER & & & & & & & \\
\hline REMARK & Spar & Ean & $02 \mathrm{exp}$ & orted & Molecule001 & & \\
\hline НЕТАТМ & 1 & $\mathrm{Na}$ & UNK & 0001 & 1.385 & -0.318 & 2.281 \\
\hline HETATM & 2 & $\mathrm{C}$ & UNK & 0001 & 1.036 & 0.704 & -0.137 \\
\hline НЕТАТМ & 3 & $\mathrm{C}$ & UNK & 0001 & 0.169 & -0.394 & -0.104 \\
\hline НЕTАТМ & 4 & $\mathrm{H}$ & UNK & 0001 & 3.123 & 0.751 & -0.427 \\
\hline НЕТАТМ & 5 & C & UNK & 0001 & 1.003 & -1.583 & -0.153 \\
\hline НЕТАТМ & 6 & $\mathrm{C}$ & UNK & 0001 & 2.287 & -1.163 & -0.215 \\
\hline НЕТАТМ & 7 & $\mathrm{~N}$ & UNK & 0001 & 2.334 & 0.217 & -0.136 \\
\hline НЕТАТМ & 8 & $\mathrm{C}$ & UNK & 0001 & 0.579 & 2.025 & -0.099 \\
\hline НЕТАТМ & 9 & $\mathrm{H}$ & UNK & 0001 & 1.254 & 2.861 & -0.137 \\
\hline НЕТАТМ & 10 & $\mathrm{C}$ & UNK & 0001 & -0.781 & 2.200 & -0.045 \\
\hline HETATM & 11 & $\mathrm{H}$ & UNK & 0001 & -1.185 & 3.196 & -0.031 \\
\hline НЕТАТМ & 12 & $\mathrm{C}$ & UNK & 0001 & -1.669 & 1.108 & -0.017 \\
\hline НЕТАТМ & 13 & $\mathrm{H}$ & UNK & 0001 & -2.725 & 1.306 & 0.017 \\
\hline НETATM & 14 & C & UNK & 0001 & -1.229 & -0.195 & -0.047 \\
\hline НЕТАТМ & 15 & $\mathrm{H}$ & UNK & 0001 & 3.189 & -1.732 & -0.316 \\
\hline НЕТАТМ & 16 & $\mathrm{H}$ & UNK & 0001 & 0.677 & -2.603 & -0.194 \\
\hline HЕTATM & 17 & C & UNK & 0001 & -2.179 & -1.366 & -0.055 \\
\hline НЕTATM & 18 & $\mathrm{H}$ & UNK & 0001 & -3.203 & -1.037 & 0.055 \\
\hline НETATM & 19 & $\mathrm{H}$ & UNK & 0001 & -1.963 & -2.063 & 0.750 \\
\hline НЕТАТМ & 20 & $\mathrm{H}$ & UNK & 0001 & -2.102 & -1.915 & -0.989 \\
\hline НЕTATM & 21 & Lig & UNK & 0001 & 1.366 & -0.444 & -0.149 \\
\hline CONECT & 1 & 21 & & & & & \\
\hline CONECT & 2 & 7 & 3 & 8 & & & \\
\hline
\end{tabular}




$\begin{array}{lrrrr}\text { CONECT } & 3 & 5 & 2 & 14 \\ \text { CONECT } & 4 & 7 & & \\ \text { CONECT } & 5 & 6 & 3 & 16 \\ \text { CONECT } & 6 & 7 & 5 & 15 \\ \text { CONECT } & 7 & 2 & 6 & 4 \\ \text { CONECT } & 8 & 9 & 2 & 10 \\ \text { CONECT } & 9 & 8 & & \\ \text { CONECT } & 10 & 11 & 8 & 12 \\ \text { CONECT } & 11 & 10 & & \\ \text { CONECT } & 12 & 13 & 10 & 14 \\ \text { CONECT } & 13 & 12 & & \\ \text { CONECT } & 14 & 12 & 3 & 17 \\ \text { CONECT } & 15 & 6 & & \\ \text { CONECT } & 16 & 5 & & \\ \text { CONECT } & 17 & 18 & 19 & 20 \\ \text { CONECT } & 18 & 17 & & \\ \text { CONECT } & 19 & 17 & & \\ \text { CONECT } & 20 & 17 & & \\ \text { CONECT } & 21 & 1 & & \\ \text { END } & & & & \end{array}$

19a. 5-Methylindole (LCH0024)

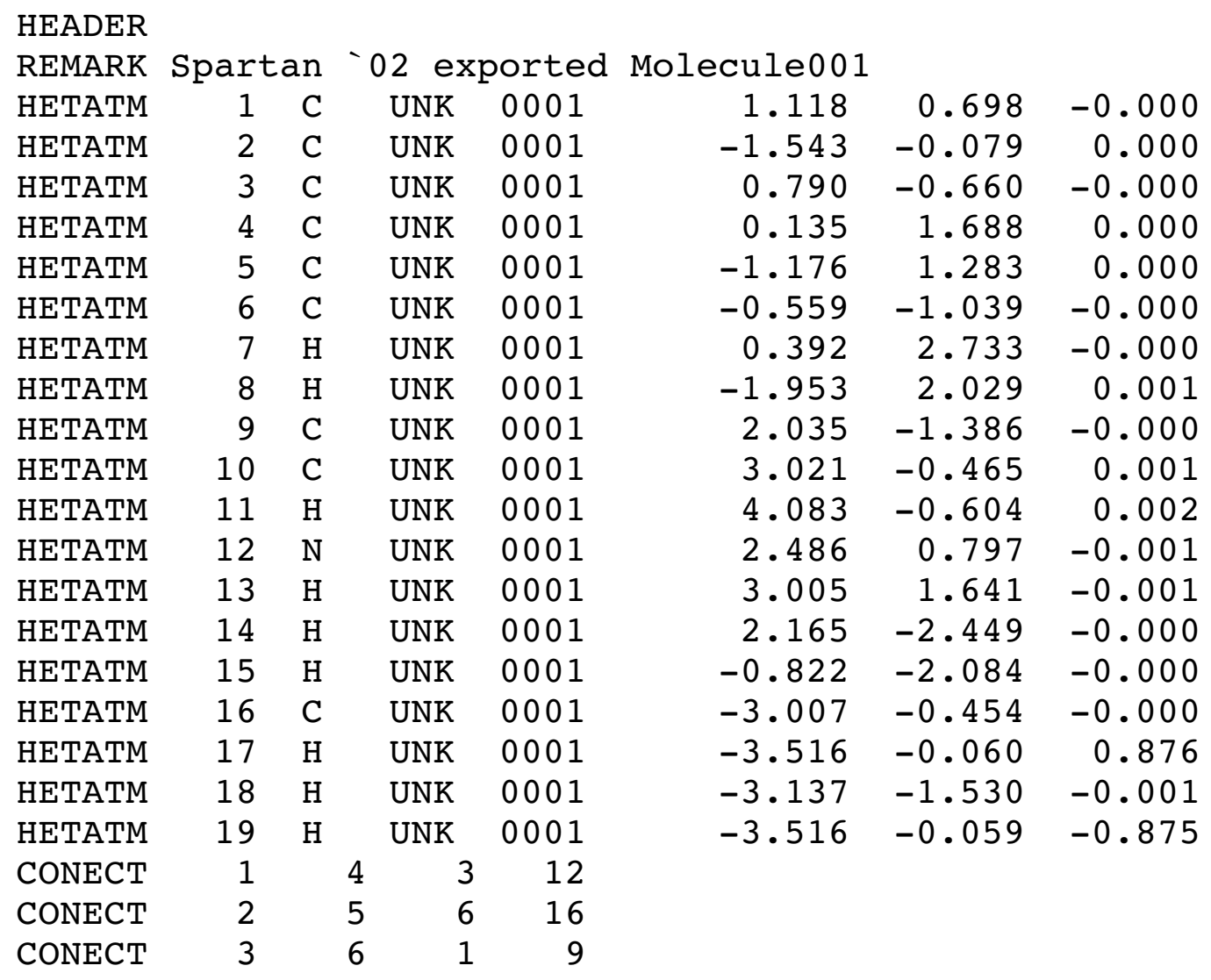




$\begin{array}{lrrrr}\text { CONECT } & 4 & 1 & 5 & 7 \\ \text { CONECT } & 5 & 4 & 2 & 8 \\ \text { CONECT } & 6 & 2 & 3 & 15 \\ \text { CONECT } & 7 & 4 & & \\ \text { CONECT } & 8 & 5 & & \\ \text { CONECT } & 9 & 3 & 10 & 14 \\ \text { CONECT } & 10 & 11 & 9 & 12 \\ \text { CONECT } & 11 & 10 & & \\ \text { CONECT } & 12 & 13 & 10 & 1 \\ \text { CONECT } & 13 & 12 & & \\ \text { CONECT } & 14 & 9 & & \\ \text { CONECT } & 15 & 6 & & \\ \text { CONECT } & 16 & 17 & 18 & 19 \\ \text { CONECT } & 17 & 16 & & \\ \text { CONECT } & 18 & 16 & & \\ \text { CONECT } & 19 & 16 & & \\ \text { END } & & & & \end{array}$

19b. $\eta^{6}-(5-M e t h y l i n d o l e) \cdot \mathrm{Na}^{+}(\mathrm{LCHO025})$

\begin{tabular}{|c|c|c|c|c|c|c|c|}
\hline \\
\hline REMARK & \multicolumn{2}{|c|}{ Spartan } & \multicolumn{2}{|c|}{02 exported } & \multicolumn{3}{|l|}{ Molecule001 } \\
\hline НЕTATM & 1 & $\mathrm{Na}$ & UNK & 0001 & -0.254 & 0.292 & -2.324 \\
\hline НЕTATM & 2 & $\mathrm{H}$ & UNK & 0001 & -0.808 & -2.101 & 0.043 \\
\hline HETATM & 3 & $\mathrm{C}$ & UNK & 0001 & -0.546 & -1.057 & 0.038 \\
\hline НЕТАТМ & 4 & $\mathrm{C}$ & UNK & 0001 & 0.152 & 1.680 & 0.105 \\
\hline НЕТАТМ & 5 & $\mathrm{C}$ & UNK & 0001 & -1.540 & -0.092 & 0.076 \\
\hline HETATM & 6 & $\mathrm{C}$ & UNK & 0001 & 0.809 & -0.676 & 0.045 \\
\hline HETATM & 7 & C & UNK & 0001 & 1.140 & 0.691 & 0.094 \\
\hline HETATM & 8 & C & UNK & 0001 & -1.170 & 1.275 & 0.095 \\
\hline HETATM & 9 & $\mathrm{H}$ & UNK & 0001 & -1.943 & 2.021 & 0.139 \\
\hline HETATM & 10 & $\mathrm{H}$ & UNK & 0001 & 0.403 & 2.725 & 0.156 \\
\hline НЕТАТМ & 11 & C & UNK & 0001 & 2.052 & -1.403 & 0.050 \\
\hline НЕТАТМ & 12 & $\mathrm{H}$ & UNK & 0001 & 2.181 & -2.465 & 0.038 \\
\hline HETATM & 13 & C & UNK & 0001 & 3.035 & -0.479 & 0.115 \\
\hline НЕТАТМ & 14 & $\mathrm{H}$ & UNK & 0001 & 4.095 & -0.620 & 0.158 \\
\hline НЕТАТМ & 15 & $\mathrm{~N}$ & UNK & 0001 & 2.504 & 0.784 & 0.121 \\
\hline HETATM & 16 & $\mathrm{H}$ & UNK & 0001 & 3.026 & 1.619 & 0.253 \\
\hline НЕТАТМ & 17 & $\mathrm{C}$ & UNK & 0001 & -3.001 & -0.476 & 0.173 \\
\hline НЕTATM & 18 & $\mathrm{H}$ & UNK & 0001 & -3.164 & -1.498 & -0.148 \\
\hline НЕТАТМ & 19 & $\mathrm{H}$ & UNK & 0001 & -3.628 & 0.173 & -0.428 \\
\hline НЕТАТМ & 20 & $\mathrm{H}$ & UNK & 0001 & -3.342 & -0.395 & 1.200 \\
\hline HETATM & 21 & Lig & UNK & 0001 & -0.193 & 0.303 & 0.076 \\
\hline CONECT & 1 & 21 & & & & & \\
\hline CONECT & 2 & 3 & 3 & & & & \\
\hline CONECT & 3 & 2 & 6 & 5 & & & \\
\hline CONECT & 4 & 7 & 8 & 10 & & & \\
\hline
\end{tabular}




$\begin{array}{lrrrr}\text { CONECT } & 5 & 8 & 3 & 17 \\ \text { CONECT } & 6 & 3 & 7 & 11 \\ \text { CONECT } & 7 & 6 & 4 & 15 \\ \text { CONECT } & 8 & 4 & 5 & 9 \\ \text { CONECT } & 9 & 8 & & \\ \text { CONECT } & 10 & 4 & & \\ \text { CONECT } & 11 & 12 & 6 & 13 \\ \text { CONECT } & 12 & 11 & & \\ \text { CONECT } & 13 & 14 & 11 & 15 \\ \text { CONECT } & 14 & 13 & & \\ \text { CONECT } & 15 & 16 & 13 & 7 \\ \text { CONECT } & 16 & 15 & & \\ \text { CONECT } & 17 & 18 & 19 & 20 \\ \text { CONECT } & 18 & 17 & & \\ \text { CONECT } & 19 & 17 & & \\ \text { CONECT } & 20 & 17 & & \\ \text { CONECT } & 21 & 1 & & \\ \text { END } & & & & \end{array}$

19c. $\eta^{5}$-(5-Methylindole) $\bullet \mathrm{Na}^{+}(\mathrm{LCH0026})$

\begin{tabular}{|c|c|c|c|c|c|c|c|}
\hline HEADER & & & & & & & \\
\hline REMARK & Spart & Ean & $02 \mathrm{exp}$ & orted & Molecule001 & & \\
\hline НЕТАТМ & 1 & $\mathrm{Na}$ & UNK & 0001 & 1.759 & -0.170 & 2.274 \\
\hline НETATM & 2 & $\mathrm{C}$ & UNK & 0001 & 1.018 & 0.716 & -0.124 \\
\hline НЕTАТМ & 3 & $\mathrm{C}$ & UNK & 0001 & 0.699 & -0.644 & -0.131 \\
\hline HETATM & 4 & $\mathrm{H}$ & UNK & 0001 & 2.891 & 1.652 & -0.387 \\
\hline НЕТАТМ & 5 & $\mathrm{C}$ & UNK & 0001 & 1.956 & -1.366 & -0.194 \\
\hline НЕTATM & 6 & $\mathrm{C}$ & UNK & 0001 & 2.942 & -0.439 & -0.228 \\
\hline НЕТАТМ & 7 & $\mathrm{~N}$ & UNK & 0001 & 2.402 & 0.828 & -0.115 \\
\hline HETATM & 8 & $\mathrm{C}$ & UNK & 0001 & 0.029 & 1.704 & -0.071 \\
\hline НЕТАТМ & 9 & $\mathrm{H}$ & UNK & 0001 & 0.276 & 2.751 & -0.078 \\
\hline НЕTАTM & 10 & C & UNK & 0001 & -1.275 & 1.284 & -0.037 \\
\hline НЕТАТМ & 11 & $\mathrm{H}$ & UNK & 0001 & -2.057 & 2.023 & -0.013 \\
\hline НЕТАТМ & 12 & $\mathrm{C}$ & UNK & 0001 & -1.638 & -0.085 & -0.041 \\
\hline НЕТАТМ & 13 & C & UNK & 0001 & -0.651 & -1.040 & -0.081 \\
\hline НЕТАТМ & 14 & $\mathrm{H}$ & UNK & 0001 & 4.000 & -0.571 & -0.331 \\
\hline HETATM & 15 & $\mathrm{H}$ & UNK & 0001 & 2.089 & -2.427 & -0.265 \\
\hline НЕТАТМ & 16 & $\mathrm{H}$ & UNK & 0001 & -0.904 & -2.086 & -0.098 \\
\hline НЕТАТМ & 17 & C & UNK & 0001 & -3.100 & -0.462 & -0.022 \\
\hline HETATM & 18 & $\mathrm{H}$ & UNK & 0001 & -3.607 & -0.022 & 0.831 \\
\hline HETATM & 19 & $\mathrm{H}$ & UNK & 0001 & -3.230 & -1.536 & 0.029 \\
\hline НЕТАТМ & 20 & $\mathrm{H}$ & UNK & 0001 & -3.600 & -0.108 & -0.918 \\
\hline HETATM & 21 & Lig & UNK & 0001 & 1.803 & -0.181 & -0.158 \\
\hline CONECT & 1 & 21 & & & & & \\
\hline CONECT & 2 & 7 & 3 & 8 & & & \\
\hline CONECT & 3 & 5 & 2 & 13 & & & \\
\hline
\end{tabular}




$\begin{array}{lrrrrr}\text { CONECT } & 4 & 7 & & & \\ \text { CONECT } & 5 & 6 & 3 & 15 & \\ \text { CONECT } & 6 & 7 & 5 & 14 & \\ \text { CONECT } & 7 & 2 & 6 & 4 & \\ \text { CONECT } & 8 & 9 & 2 & 10 \\ \text { CONECT } & 9 & 8 & & & \\ \text { CONECT } & 10 & 11 & 8 & 12 & \\ \text { CONECT } & 11 & 10 & & & \\ \text { CONECT } & 12 & 10 & 13 & 17 & \\ \text { CONECT } & 13 & 12 & 3 & 16 & \\ \text { CONECT } & 14 & 6 & & & \\ \text { CONECT } & 15 & 5 & & & \\ \text { CONECT } & 16 & 13 & & & \\ \text { CONECT } & 17 & 18 & 19 & 20 & 12 \\ \text { CONECT } & 18 & 17 & & & \\ \text { CONECT } & 19 & 17 & & & \\ \text { CONECT } & 20 & 17 & & & \\ \text { CONECT } & 21 & 1 & & & \\ \text { END } & & & & & \end{array}$

20a. 6-Methylindole (LCH0028)

\begin{tabular}{|c|c|c|c|c|c|c|c|}
\hline HEADER & & & & & & & \\
\hline REMARK & Spart & & $02 \mathrm{ex}$ & orted & Molecule001 & & \\
\hline HETATM & 1 & $\mathrm{C}$ & UNK & 0001 & 0.782 & -0.612 & -0.000 \\
\hline HETATM & 2 & $\mathrm{C}$ & UNK & 0001 & -1.180 & 1.297 & -0.000 \\
\hline НЕTATM & 3 & $\mathrm{C}$ & UNK & 0001 & 1.144 & 0.737 & -0.000 \\
\hline HETATM & 4 & $\mathrm{C}$ & UNK & 0001 & -0.551 & -1.028 & -0.000 \\
\hline НЕТАТМ & 5 & $\mathrm{C}$ & UNK & 0001 & -1.537 & -0.068 & 0.000 \\
\hline НЕТАТМ & 6 & $\mathrm{C}$ & UNK & 0001 & 0.130 & 1.702 & -0.000 \\
\hline НЕTATM & 7 & $\mathrm{H}$ & UNK & 0001 & -0.802 & -2.075 & -0.000 \\
\hline HETATM & 8 & $\mathrm{C}$ & UNK & 0001 & 2.585 & 0.782 & 0.000 \\
\hline HETATM & 9 & $\mathrm{C}$ & UNK & 0001 & 3.012 & -0.497 & 0.000 \\
\hline НЕTATM & 10 & $\mathrm{H}$ & UNK & 0001 & 4.010 & -0.884 & 0.000 \\
\hline HETATM & 11 & $\mathrm{~N}$ & UNK & 0001 & 1.935 & -1.351 & -0.001 \\
\hline HETATM & 12 & $\mathrm{H}$ & UNK & 0001 & 1.988 & -2.340 & 0.000 \\
\hline HETATM & 13 & $\mathrm{H}$ & UNK & 0001 & 3.206 & 1.654 & 0.000 \\
\hline HETATM & 14 & $\mathrm{H}$ & UNK & 0001 & 0.375 & 2.751 & -0.000 \\
\hline НETATM & 15 & $\mathrm{H}$ & UNK & 0001 & -1.963 & 2.037 & 0.000 \\
\hline НЕТАТМ & 16 & $\mathrm{C}$ & UNK & 0001 & -2.998 & -0.454 & 0.000 \\
\hline HETATM & 17 & $\mathrm{H}$ & UNK & 0001 & -3.123 & -1.531 & -0.000 \\
\hline HETATM & 18 & $\mathrm{H}$ & UNK & 0001 & -3.507 & -0.059 & -0.875 \\
\hline НЕТАТМ & 19 & $\mathrm{H}$ & UNK & 0001 & -3.506 & -0.060 & 0.876 \\
\hline CONECT & 1 & & 3 & 11 & & & \\
\hline CONECT & 2 & & 5 & 15 & & & \\
\hline CONECT & 3 & & 6 & 8 & & & \\
\hline CONECT & 4 & & 1 & 7 & & & \\
\hline
\end{tabular}




$\begin{array}{lrrrrr}\text { CONECT } & 5 & 4 & 2 & 16 & \\ \text { CONECT } & 6 & 2 & 3 & 14 & \\ \text { CONECT } & 7 & 4 & & & \\ \text { CONECT } & 8 & 3 & 9 & 13 & \\ \text { CONECT } & 9 & 10 & 8 & 11 \\ \text { CONECT } & 10 & 9 & & & \\ \text { CONECT } & 11 & 12 & 9 & 1 & \\ \text { CONECT } & 12 & 11 & & & \\ \text { CONECT } & 13 & 8 & & & \\ \text { CONECT } & 14 & 6 & & & \\ \text { CONECT } & 15 & 2 & & & \\ \text { CONECT } & 16 & 17 & 18 & 19 & 5 \\ \text { CONECT } & 17 & 16 & & & \\ \text { CONECT } & 18 & 16 & & & \\ \text { CONECT } & 19 & 16 & & & \\ \text { END } & & & & \end{array}$

20b. $\eta^{6}-(6-M e t h y l i n d o l e) \cdot \mathrm{Na}^{+}(\mathrm{LCHO029})$

\begin{tabular}{|c|c|c|c|c|c|c|c|}
\hline HEADER & & & & & & & \\
\hline REMARK & Spart & Ean & $02 \mathrm{exp}$ & oorted & Molecule001 & & \\
\hline HETATM & 1 & $\mathrm{Na}$ & UNK & 0001 & -0.208 & -0.554 & -2.303 \\
\hline НЕTATM & 2 & $\mathrm{H}$ & UNK & 0001 & 0.390 & -2.725 & 0.243 \\
\hline HETATM & 3 & $\mathrm{C}$ & UNK & 0001 & 0.146 & -1.680 & 0.160 \\
\hline НЕТАТМ & 4 & $\mathrm{C}$ & UNK & 0001 & -0.542 & 1.056 & 0.008 \\
\hline НЕТАТМ & 5 & C & UNK & 0001 & -1.175 & -1.275 & 0.138 \\
\hline HETATM & 6 & $\mathrm{C}$ & UNK & 0001 & 1.163 & -0.714 & 0.104 \\
\hline HETATM & 7 & $\mathrm{C}$ & UNK & 0001 & 0.797 & 0.645 & 0.044 \\
\hline HETATM & 8 & $\mathrm{C}$ & UNK & 0001 & -1.537 & 0.093 & 0.072 \\
\hline НЕТАТМ & 9 & $\mathrm{H}$ & UNK & 0001 & -0.796 & 2.102 & -0.016 \\
\hline НЕТАТМ & 10 & C & UNK & 0001 & 2.604 & -0.755 & 0.133 \\
\hline HETATM & 11 & $\mathrm{H}$ & UNK & 0001 & 3.225 & -1.624 & 0.198 \\
\hline НЕТАТМ & 12 & $\mathrm{C}$ & UNK & 0001 & 3.023 & 0.526 & 0.102 \\
\hline НЕТАТМ & 13 & $\mathrm{H}$ & UNK & 0001 & 4.020 & 0.916 & 0.128 \\
\hline НЕTATM & 14 & $\mathrm{~N}$ & UNK & 0001 & 1.948 & 1.378 & 0.027 \\
\hline НЕТАТМ & 15 & $\mathrm{H}$ & UNK & 0001 & 2.003 & 2.369 & 0.082 \\
\hline НЕТАТМ & 16 & $\mathrm{H}$ & UNK & 0001 & -1.954 & -2.013 & 0.215 \\
\hline НЕTATM & 17 & $\mathrm{C}$ & UNK & 0001 & -2.995 & 0.489 & 0.144 \\
\hline НЕTATM & 18 & $\mathrm{H}$ & UNK & 0001 & -3.621 & -0.184 & -0.430 \\
\hline НЕТАТМ & 19 & $\mathrm{H}$ & UNK & 0001 & -3.152 & 1.496 & -0.222 \\
\hline НЕТАТМ & 20 & $\mathrm{H}$ & UNK & 0001 & -3.339 & 0.454 & 1.173 \\
\hline НЕTATM & 21 & Lig & UNK & 0001 & -0.191 & -0.312 & 0.088 \\
\hline CONECT & 1 & 21 & & & & & \\
\hline CONECT & 2 & 3 & & & & & \\
\hline CONECT & 3 & 2 & 6 & 5 & & & \\
\hline CONECT & 4 & 7 & 8 & 9 & & & \\
\hline CONECT & 5 & 8 & 3 & 16 & & & \\
\hline
\end{tabular}




$\begin{array}{lrrrrl}\text { CONECT } & 6 & 3 & 7 & 10 \\ \text { CONECT } & 7 & 6 & 4 & 14 \\ \text { CONECT } & 8 & 4 & 5 & 17 \\ \text { CONECT } & 9 & 4 & & & \\ \text { CONECT } & 10 & 11 & 6 & 12 \\ \text { CONECT } & 11 & 10 & & & \\ \text { CONECT } & 12 & 13 & 10 & 14 \\ \text { CONECT } & 13 & 12 & & & \\ \text { CONECT } & 14 & 15 & 12 & 7 \\ \text { CONECT } & 15 & 14 & & & \\ \text { CONECT } & 16 & 5 & & & \\ \text { CONECT } & 17 & 18 & 19 & 20 \\ \text { CONECT } & 18 & 17 & & & \\ \text { CONECT } & 19 & 17 & & \\ \text { CONECT } & 20 & 17 & & \\ \text { CONECT } & 21 & 1 & & \\ \text { END } & & & & \end{array}$

20c. $\eta^{5}$-(6-Methylindole $) \cdot \mathrm{Na}^{+}(\mathrm{LCHO030})$

\begin{tabular}{|c|c|c|c|c|c|c|c|}
\hline HEADER & & & & & & & \\
\hline REMARK & Spart & $=a n$ & $02 \mathrm{exp}$ & orted & Molecule001 & & \\
\hline НЕTATM & 1 & $\mathrm{Na}$ & UNK & 0001 & 1.776 & 0.143 & 2.272 \\
\hline НЕTATM & 2 & C & UNK & 0001 & 0.688 & 0.603 & -0.120 \\
\hline HETATM & 3 & C & UNK & 0001 & 1.043 & -0.748 & -0.135 \\
\hline HETATM & 4 & $\mathrm{H}$ & UNK & 0001 & 1.906 & 2.306 & -0.364 \\
\hline НЕТАТМ & 5 & $\mathrm{C}$ & UNK & 0001 & 2.492 & -0.802 & -0.204 \\
\hline HETATM & 6 & $\mathrm{C}$ & UNK & 0001 & 2.931 & 0.478 & -0.228 \\
\hline HETATM & 7 & $\mathrm{~N}$ & UNK & 0001 & 1.858 & 1.345 & -0.107 \\
\hline HETATM & 8 & C & UNK & 0001 & -0.643 & 1.031 & -0.062 \\
\hline HETATM & 9 & $\mathrm{H}$ & UNK & 0001 & -0.888 & 2.079 & -0.064 \\
\hline HETATM & 10 & $\mathrm{C}$ & UNK & 0001 & -1.632 & 0.074 & -0.037 \\
\hline НЕТАТМ & 11 & C & UNK & 0001 & -1.280 & -1.298 & -0.048 \\
\hline HETATM & 12 & $\mathrm{C}$ & UNK & 0001 & 0.022 & -1.717 & -0.094 \\
\hline HETATM & 13 & $\mathrm{H}$ & UNK & 0001 & 3.927 & 0.857 & -0.329 \\
\hline НЕТАТМ & 14 & $\mathrm{H}$ & UNK & 0001 & 3.104 & -1.678 & -0.283 \\
\hline НЕTATM & 15 & $\mathrm{H}$ & UNK & 0001 & 0.260 & -2.765 & -0.118 \\
\hline НETATM & 16 & $\mathrm{H}$ & UNK & 0001 & -2.067 & -2.030 & -0.030 \\
\hline НЕТАТМ & 17 & $\mathrm{C}$ & UNK & 0001 & -3.091 & 0.461 & -0.014 \\
\hline HETATM & 18 & $\mathrm{H}$ & UNK & 0001 & -3.602 & -0.000 & 0.825 \\
\hline НЕТАТМ & 19 & $\mathrm{H}$ & UNK & 0001 & -3.586 & 0.128 & -0.921 \\
\hline HETATM & 20 & $\mathrm{H}$ & UNK & 0001 & -3.217 & 1.533 & 0.061 \\
\hline НETATM & 21 & Lig & UNK & 0001 & 1.802 & 0.175 & -0.159 \\
\hline CONECT & 1 & 21 & & & & & \\
\hline CONECT & 2 & 7 & 3 & 8 & & & \\
\hline CONECT & 3 & 5 & 2 & 12 & & & \\
\hline CONECT & 4 & 7 & 7 & & & & \\
\hline
\end{tabular}




$\begin{array}{lrrrrr}\text { CONECT } & 5 & 6 & 3 & 14 & \\ \text { CONECT } & 6 & 7 & 5 & 13 & \\ \text { CONECT } & 7 & 2 & 6 & 4 & \\ \text { CONECT } & 8 & 9 & 2 & 10 & \\ \text { CONECT } & 9 & 8 & & & \\ \text { CONECT } & 10 & 8 & 11 & 17 & \\ \text { CONECT } & 11 & 10 & 12 & 16 & \\ \text { CONECT } & 12 & 11 & 3 & 15 & \\ \text { CONECT } & 13 & 6 & & & \\ \text { CONECT } & 14 & 5 & & & \\ \text { CONECT } & 15 & 12 & & & \\ \text { CONECT } & 16 & 11 & & & \\ \text { CONECT } & 17 & 18 & 19 & 20 & 10 \\ \text { CONECT } & 18 & 17 & & & \\ \text { CONECT } & 19 & 17 & & & \\ \text { CONECT } & 20 & 17 & & & \\ \text { CONECT } & 21 & 1 & & & \\ \text { END } & & & & & \end{array}$

\section{1a. 7-Methylindole (LCH0032)}

\begin{tabular}{|c|c|c|c|c|c|c|c|}
\hline HEADER & & & & & & & \\
\hline REMARK & Spart & & 02 ex & ported & Molecule001 & & \\
\hline HETATM & 1 & $\mathrm{C}$ & UNK & 0001 & -0.241 & -0.371 & -0.000 \\
\hline НЕТАТМ & 2 & $\mathrm{C}$ & UNK & 0001 & 0.660 & 2.215 & 0.000 \\
\hline НЕТАТМ & 3 & $\mathrm{C}$ & UNK & 0001 & -1.163 & 0.680 & 0.000 \\
\hline НЕТАТМ & 4 & $\mathrm{C}$ & UNK & 0001 & 1.145 & -0.171 & -0.000 \\
\hline HETATM & 5 & $\mathrm{C}$ & UNK & 0001 & 1.565 & 1.139 & 0.000 \\
\hline НЕТАТМ & 6 & $\mathrm{C}$ & UNK & 0001 & -0.694 & 1.999 & 0.000 \\
\hline НЕТАТМ & 7 & $\mathrm{C}$ & UNK & 0001 & -2.474 & 0.082 & -0.000 \\
\hline HETATM & 8 & $\mathrm{C}$ & UNK & 0001 & -2.292 & -1.254 & -0.000 \\
\hline НЕТАТМ & 9 & $\mathrm{H}$ & UNK & 0001 & -3.018 & -2.042 & -0.001 \\
\hline НЕТАТМ & 10 & $\mathrm{~N}$ & UNK & 0001 & -0.952 & -1.546 & -0.002 \\
\hline НЕТАТМ & 11 & $\mathrm{H}$ & UNK & 0001 & -0.566 & -2.458 & 0.006 \\
\hline НЕТАТМ & 12 & $\mathrm{H}$ & UNK & 0001 & -3.416 & 0.590 & -0.001 \\
\hline НЕTATM & 13 & $\mathrm{H}$ & UNK & 0001 & -1.384 & 2.826 & -0.000 \\
\hline НЕТАТМ & 14 & $\mathrm{H}$ & UNK & 0001 & 1.045 & 3.220 & 0.001 \\
\hline НЕТАТМ & 15 & $\mathrm{H}$ & UNK & 0001 & 2.621 & 1.349 & 0.000 \\
\hline НЕТАТМ & 16 & $\mathrm{C}$ & UNK & 0001 & 2.103 & -1.336 & -0.001 \\
\hline НЕТАТМ & 17 & $\mathrm{H}$ & UNK & 0001 & 1.966 & -1.963 & 0.877 \\
\hline HETATM & 18 & $\mathrm{H}$ & UNK & 0001 & 3.130 & -0.993 & -0.002 \\
\hline НЕТАТМ & 19 & $\mathrm{H}$ & UNK & 0001 & 1.963 & -1.964 & -0.877 \\
\hline CONECT & 1 & & 3 & 10 & & & \\
\hline CONECT & 2 & & 5 & 14 & & & \\
\hline CONECT & 3 & & 6 & 7 & & & \\
\hline CONECT & 4 & & 1 & 16 & & & \\
\hline CONECT & 5 & & 4 & 15 & & & \\
\hline
\end{tabular}




\begin{tabular}{|c|c|c|c|c|}
\hline CONECT & 6 & 2 & 3 & 13 \\
\hline CONECT & 7 & 3 & 8 & 12 \\
\hline CONECT & 8 & 9 & 7 & 10 \\
\hline CONECT & 9 & 8 & & \\
\hline CONECT & 10 & 11 & 8 & 1 \\
\hline CONECT & 11 & 10 & & \\
\hline CONECT & 12 & 7 & & \\
\hline CONECT & 13 & 6 & & \\
\hline CONECT & 14 & 2 & & \\
\hline CONECT & 15 & 5 & & \\
\hline CONECT & 16 & 17 & 18 & 19 \\
\hline CONECT & 17 & 16 & & \\
\hline CONECT & 18 & 16 & & \\
\hline CONECT & 19 & 16 & & \\
\hline END & & & & \\
\hline
\end{tabular}

\section{1b. $\eta^{6}$-(7-Methylindole) $\bullet \mathrm{Na}^{+}(\mathrm{LCHO033})$}

\begin{tabular}{|c|c|c|c|c|c|c|c|}
\hline \multicolumn{8}{|l|}{ HEADER } \\
\hline REMARK & Spart & $\tan$ & $02 \mathrm{ex}$ & ported & Molecule001 & & \\
\hline HETATM & 1 & $\mathrm{Na}$ & UNK & 0001 & -0.208 & -1.210 & -2.248 \\
\hline HETATM & 2 & $\mathrm{H}$ & UNK & 0001 & -1.072 & -3.141 & 0.343 \\
\hline HETATM & 3 & $\mathrm{C}$ & UNK & 0001 & -0.677 & -2.147 & 0.231 \\
\hline HETATM & 4 & $\mathrm{C}$ & UNK & 0001 & 0.257 & 0.438 & 0.051 \\
\hline НЕTATM & 5 & $\mathrm{C}$ & UNK & 0001 & -1.574 & -1.059 & 0.152 \\
\hline HETATM & 6 & $\mathrm{C}$ & UNK & 0001 & 0.688 & -1.947 & 0.209 \\
\hline HETATM & 7 & $\mathrm{C}$ & UNK & 0001 & 1.174 & -0.631 & 0.107 \\
\hline HETATM & 8 & $\mathrm{C}$ & UNK & 0001 & -1.137 & 0.253 & 0.068 \\
\hline НЕТАТМ & 9 & $\mathrm{H}$ & UNK & 0001 & -2.631 & -1.255 & 0.201 \\
\hline НЕTATM & 10 & $\mathrm{H}$ & UNK & 0001 & 1.367 & -2.777 & 0.301 \\
\hline HETATM & 11 & $\mathrm{C}$ & UNK & 0001 & 2.490 & -0.050 & 0.086 \\
\hline НЕТАТМ & 12 & $\mathrm{H}$ & UNK & 0001 & 3.426 & -0.567 & 0.133 \\
\hline НЕTATM & 13 & $\mathrm{C}$ & UNK & 0001 & 2.318 & 1.288 & 0.034 \\
\hline HETATM & 14 & $\mathrm{H}$ & UNK & 0001 & 3.053 & 2.067 & 0.022 \\
\hline НЕTATM & 15 & $\mathrm{~N}$ & UNK & 0001 & 0.983 & 1.595 & -0.005 \\
\hline НЕTATM & 16 & $\mathrm{H}$ & UNK & 0001 & 0.614 & 2.516 & 0.036 \\
\hline НЕTATM & 17 & $\mathrm{C}$ & UNK & 0001 & -2.085 & 1.428 & 0.065 \\
\hline НЕТАТМ & 18 & $\mathrm{H}$ & UNK & 0001 & -3.112 & 1.099 & -0.025 \\
\hline НЕТАТМ & 19 & $\mathrm{H}$ & UNK & 0001 & -1.878 & 2.111 & -0.753 \\
\hline НЕTATM & 20 & $\mathrm{H}$ & UNK & 0001 & -1.997 & 1.987 & 0.992 \\
\hline HETATM & 21 & Lig & UNK & 0001 & -0.211 & -0.849 & 0.136 \\
\hline CONECT & 1 & 21 & & & & & \\
\hline CONECT & 2 & 3 & & & & & \\
\hline CONECT & 3 & 2 & 6 & 5 & & & \\
\hline CONECT & 4 & 7 & 8 & 15 & & & \\
\hline CONECT & 5 & 8 & 3 & 9 & & & \\
\hline CONECT & 6 & 3 & 7 & 10 & & & \\
\hline
\end{tabular}




$\begin{array}{lrrrrr}\text { CONECT } & 7 & 6 & 4 & 11 & \\ \text { CONECT } & 8 & 4 & 5 & 17 & \\ \text { CONECT } & 9 & 5 & & & \\ \text { CONECT } & 10 & 6 & & & \\ \text { CONECT } & 11 & 12 & 7 & 13 & \\ \text { CONECT } & 12 & 11 & & & \\ \text { CONECT } & 13 & 14 & 11 & 15 & \\ \text { CONECT } & 14 & 13 & & & \\ \text { CONECT } & 15 & 16 & 13 & 4 & \\ \text { CONECT } & 16 & 15 & & & \\ \text { CONECT } & 17 & 18 & 19 & 20 & 8 \\ \text { CONECT } & 18 & 17 & & & \\ \text { CONECT } & 19 & 17 & & & \\ \text { CONECT } & 20 & 17 & & & \\ \text { CONECT } & 21 & 1 & & & \\ \text { END } & & & & & \end{array}$

\section{1c. $\eta^{5}$-(7-Methylindole) $\bullet \mathrm{Na}^{+}(\mathrm{LCHO034})$}

\begin{tabular}{|c|c|c|c|c|c|c|c|}
\hline \multicolumn{8}{|l|}{ HEADER } \\
\hline REMARK & Spart & $\tan$ & $02 \mathrm{exp}$ & ported & Molecule001 & & \\
\hline HETATM & 1 & $\mathrm{Na}$ & UNK & 0001 & 1.370 & 0.255 & 2.287 \\
\hline НЕTATM & 2 & $\mathrm{H}$ & UNK & 0001 & 3.331 & -0.677 & -0.315 \\
\hline НЕTATM & 3 & $\mathrm{C}$ & UNK & 0001 & 2.405 & -0.148 & -0.217 \\
\hline НЕTATM & 4 & $\mathrm{C}$ & UNK & 0001 & 2.254 & 1.197 & -0.211 \\
\hline НЕTATM & 5 & $\mathrm{H}$ & UNK & 0001 & 2.995 & 1.967 & -0.299 \\
\hline НЕTATM & 6 & $\mathrm{~N}$ & UNK & 0001 & 0.920 & 1.522 & -0.079 \\
\hline HETATM & 7 & $\mathrm{H}$ & UNK & 0001 & 0.555 & 2.420 & -0.303 \\
\hline НЕTATM & 8 & $\mathrm{C}$ & UNK & 0001 & 0.175 & 0.351 & -0.106 \\
\hline НЕTATM & 9 & $\mathrm{C}$ & UNK & 0001 & 1.073 & -0.721 & -0.153 \\
\hline НЕTATM & 10 & $\mathrm{C}$ & UNK & 0001 & 0.580 & -2.037 & -0.122 \\
\hline НЕTATM & 11 & $\mathrm{H}$ & UNK & 0001 & 1.248 & -2.878 & -0.168 \\
\hline HETATM & 12 & $\mathrm{C}$ & UNK & 0001 & -0.778 & -2.215 & -0.059 \\
\hline НЕTATM & 13 & $\mathrm{H}$ & UNK & 0001 & -1.187 & -3.208 & -0.048 \\
\hline НЕTATM & 14 & $\mathrm{C}$ & UNK & 0001 & -1.659 & -1.117 & -0.025 \\
\hline НЕTATM & 15 & $\mathrm{H}$ & UNK & 0001 & -2.717 & -1.308 & 0.010 \\
\hline НЕTATM & 16 & $\mathrm{C}$ & UNK & 0001 & -1.219 & 0.187 & -0.044 \\
\hline НЕTATM & 17 & $\mathrm{C}$ & UNK & 0001 & -2.153 & 1.372 & -0.036 \\
\hline HETATM & 18 & $\mathrm{H}$ & UNK & 0001 & -2.085 & 1.928 & -0.967 \\
\hline НЕTATM & 19 & $\mathrm{H}$ & UNK & 0001 & -1.928 & 2.059 & 0.776 \\
\hline НЕTATM & 20 & $\mathrm{H}$ & UNK & 0001 & -3.180 & 1.052 & 0.080 \\
\hline НЕTATM & 21 & Lig & UNK & 0001 & 1.365 & 0.440 & -0.153 \\
\hline CONECT & 1 & 21 & & & & & \\
\hline CONECT & 2 & 3 & & & & & \\
\hline CONECT & 3 & 2 & 9 & 4 & & & \\
\hline CONECT & 4 & 6 & 3 & 5 & & & \\
\hline CONECT & 5 & & & & & & \\
\hline
\end{tabular}




$\begin{array}{lrrrrr}\text { CONECT } & 6 & 8 & 4 & 7 & \\ \text { CONECT } & 7 & 6 & & & \\ \text { CONECT } & 8 & 9 & 6 & 16 & \\ \text { CONECT } & 9 & 3 & 8 & 10 & \\ \text { CONECT } & 10 & 11 & 9 & 12 & \\ \text { CONECT } & 11 & 10 & & & \\ \text { CONECT } & 12 & 13 & 10 & 14 & \\ \text { CONECT } & 13 & 12 & & & \\ \text { CONECT } & 14 & 15 & 12 & 16 & \\ \text { CONECT } & 15 & 14 & & & \\ \text { CONECT } & 16 & 14 & 8 & 17 & \\ \text { CONECT } & 17 & 18 & 19 & 20 & 16 \\ \text { CONECT } & 18 & 17 & & & \\ \text { CONECT } & 19 & 17 & & & \\ \text { CONECT } & 20 & 17 & & & \\ \text { CONECT } & 21 & 1 & & & \\ \text { END } & & & & & \end{array}$




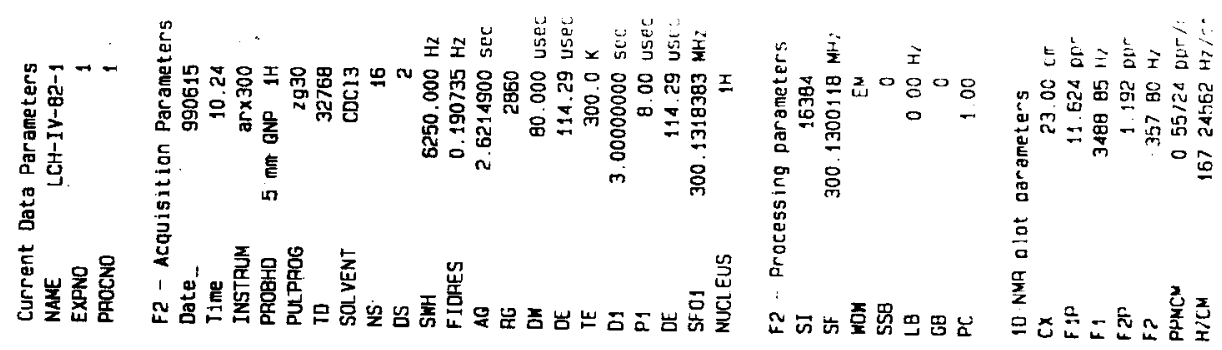

000.0

$852^{\circ} \cdot$

$2 \angle D^{\circ}$

EBT.

$\angle 2 G^{\circ} \mathrm{L}$

b89' I

s29.

$\nabla 59.1$

$1 \angle 9.1-$

$\angle 89$.

$862 \cdot 5$

I9P $\angle$

O6E $\angle$

टEE ' $\angle$

EOt $L$

SOt $L 7$

IDO

$99 \sigma^{\circ} L$

$890^{\circ} \angle$

E6t $\angle$

टाL'L

GI $L \angle$

$\angle E L ' \angle$

$O D L L$

$99 \angle \cdot$

$8 \angle L \angle L$

$095 \cdot 8-$

$88+8$

टDC OI

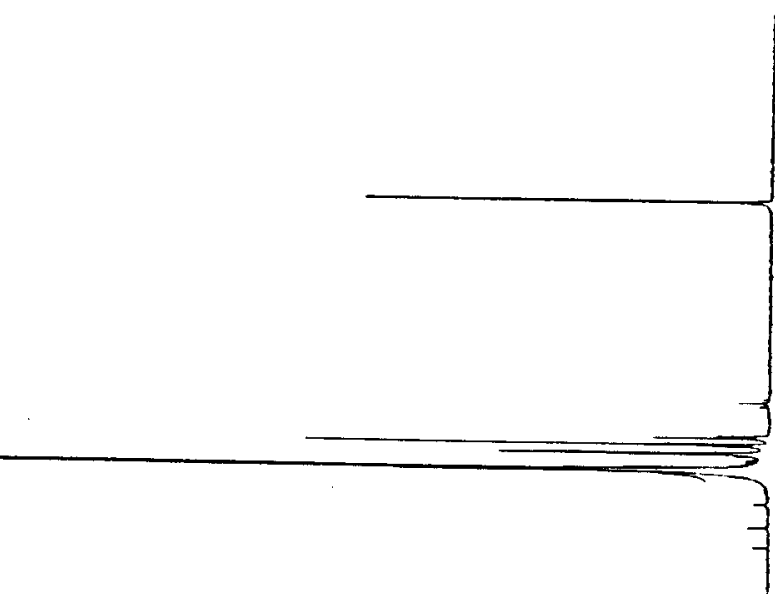

( 


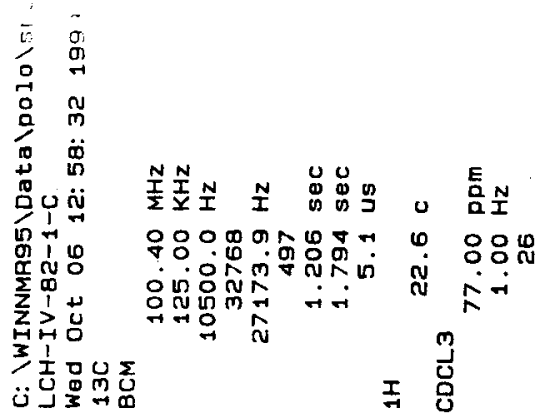

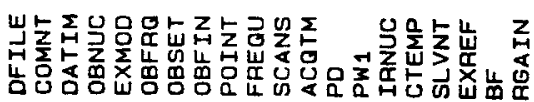

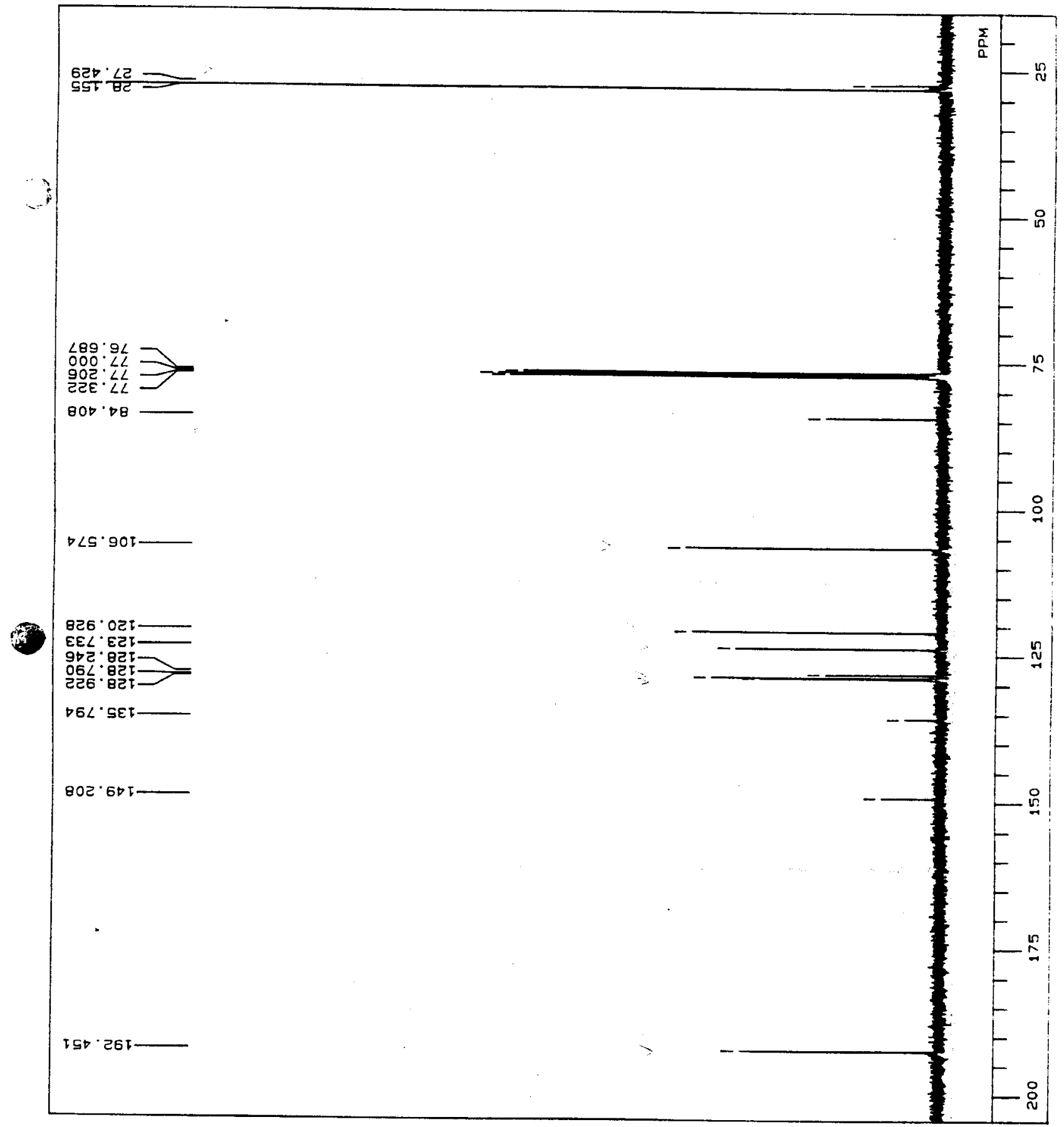




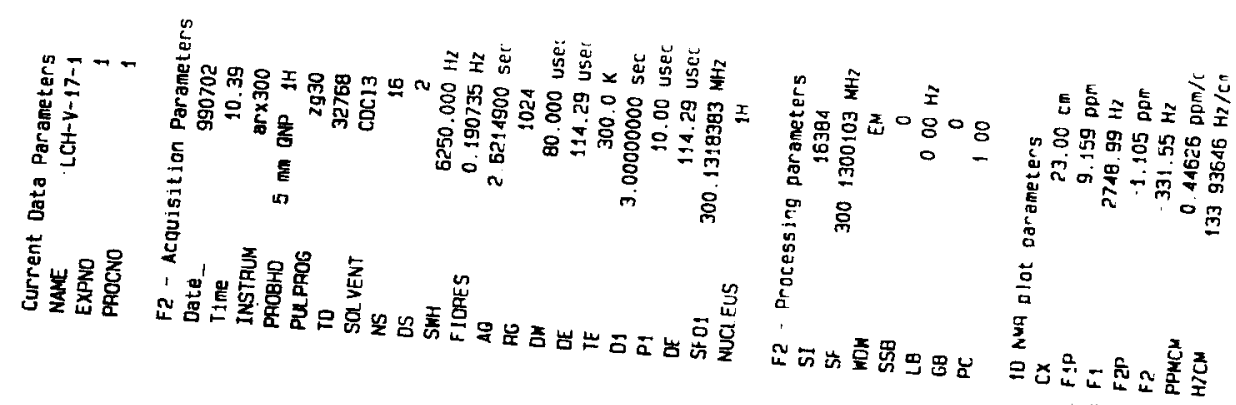

$000 \%$

$9 \angle 9 \cdot$

BL $\angle$.

$988^{\circ} \cdot$

150.2

820

I88 $\varepsilon$

$\varepsilon-$

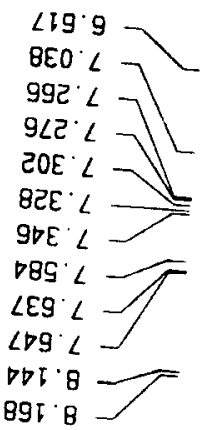

wod

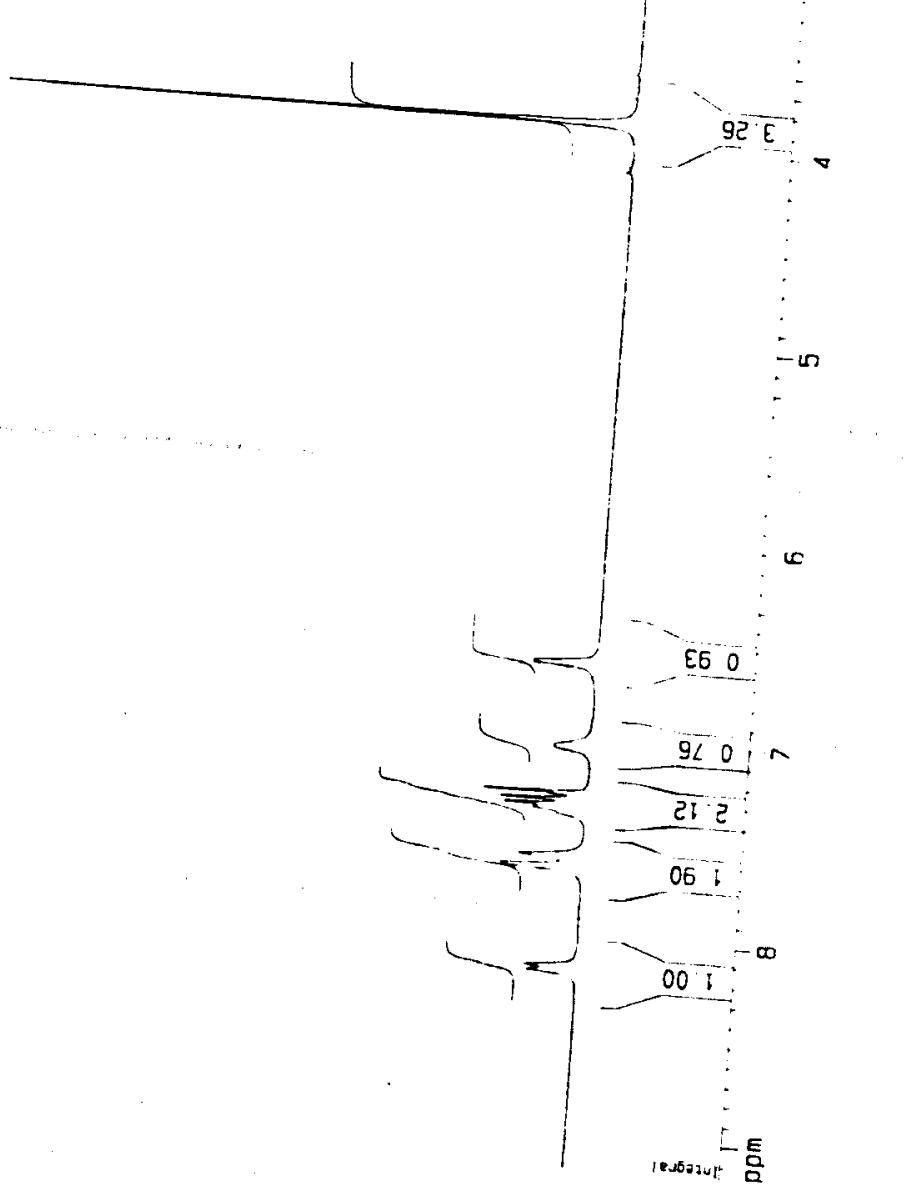




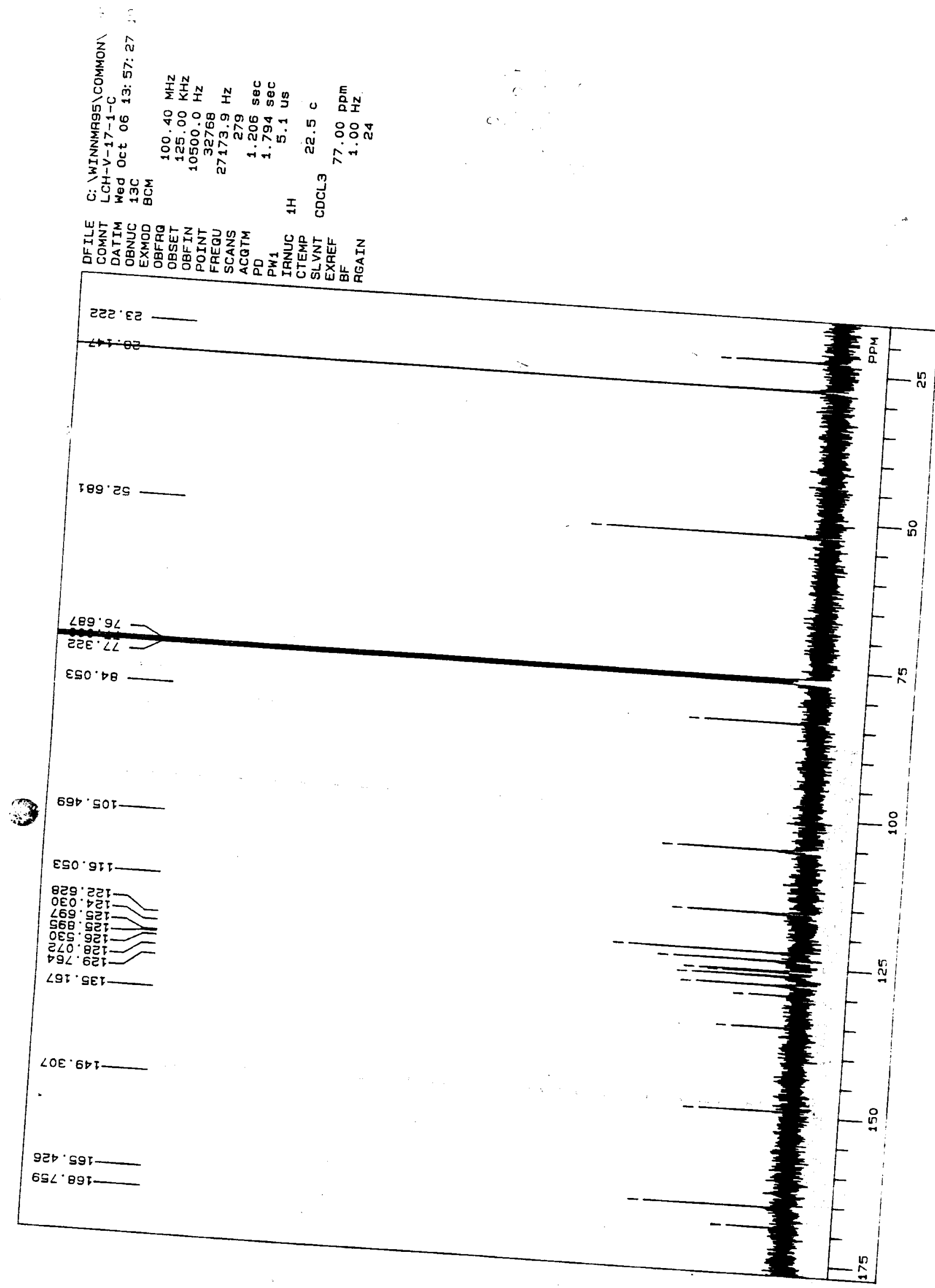




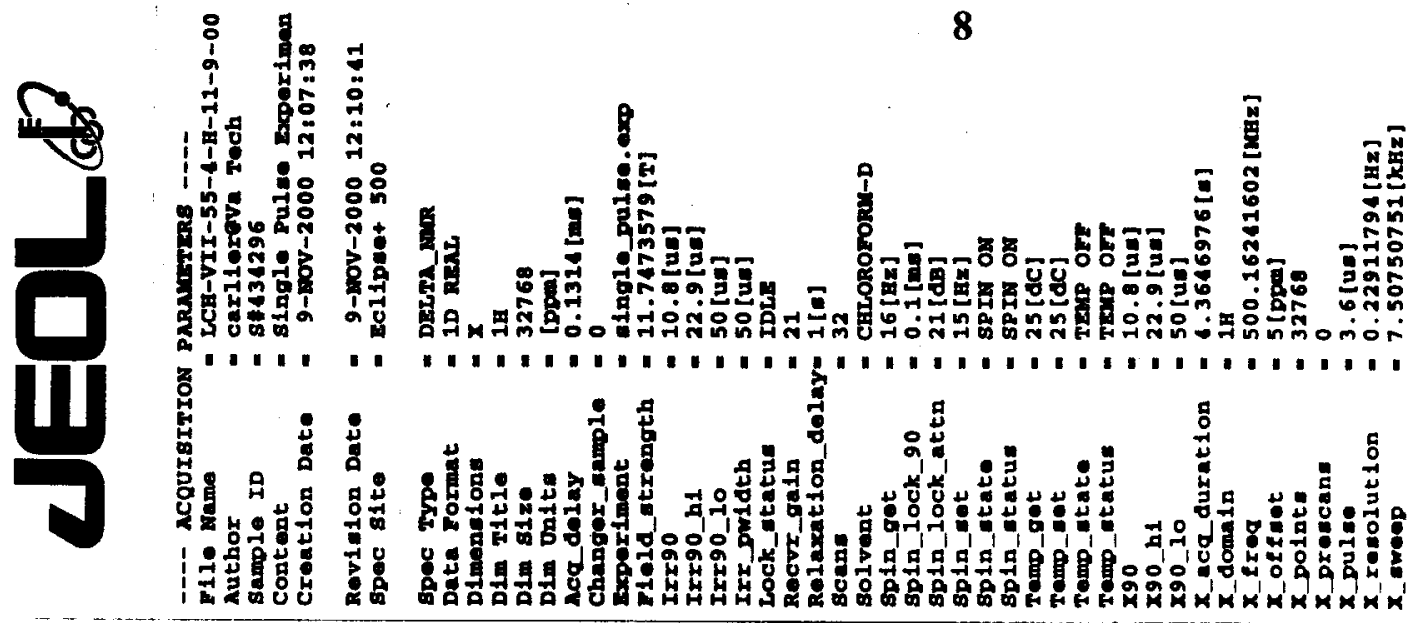

$\$ 8966^{\circ} 0$

市

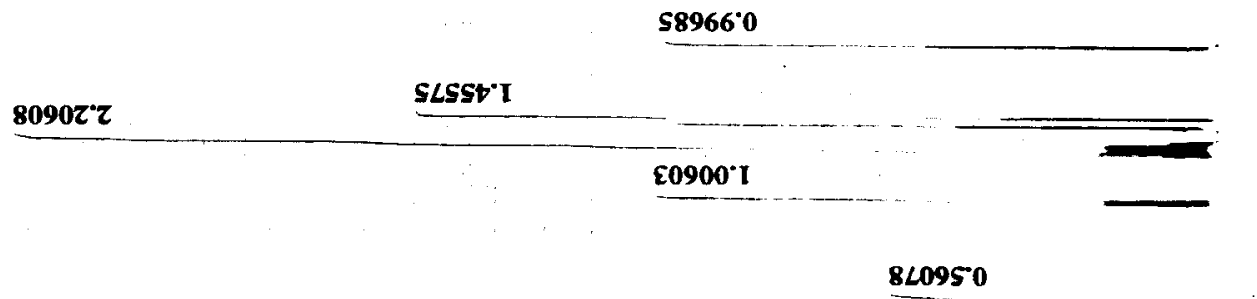

e $53<8^{\circ}$

209T:L

i: $11 \% L$ 9SOE. $L$ zItr. $L$ $\angle \angle 69^{\circ} \mathrm{L}$

웅

LEET'8

I

$0<99^{\circ} 8$ 5889.8 


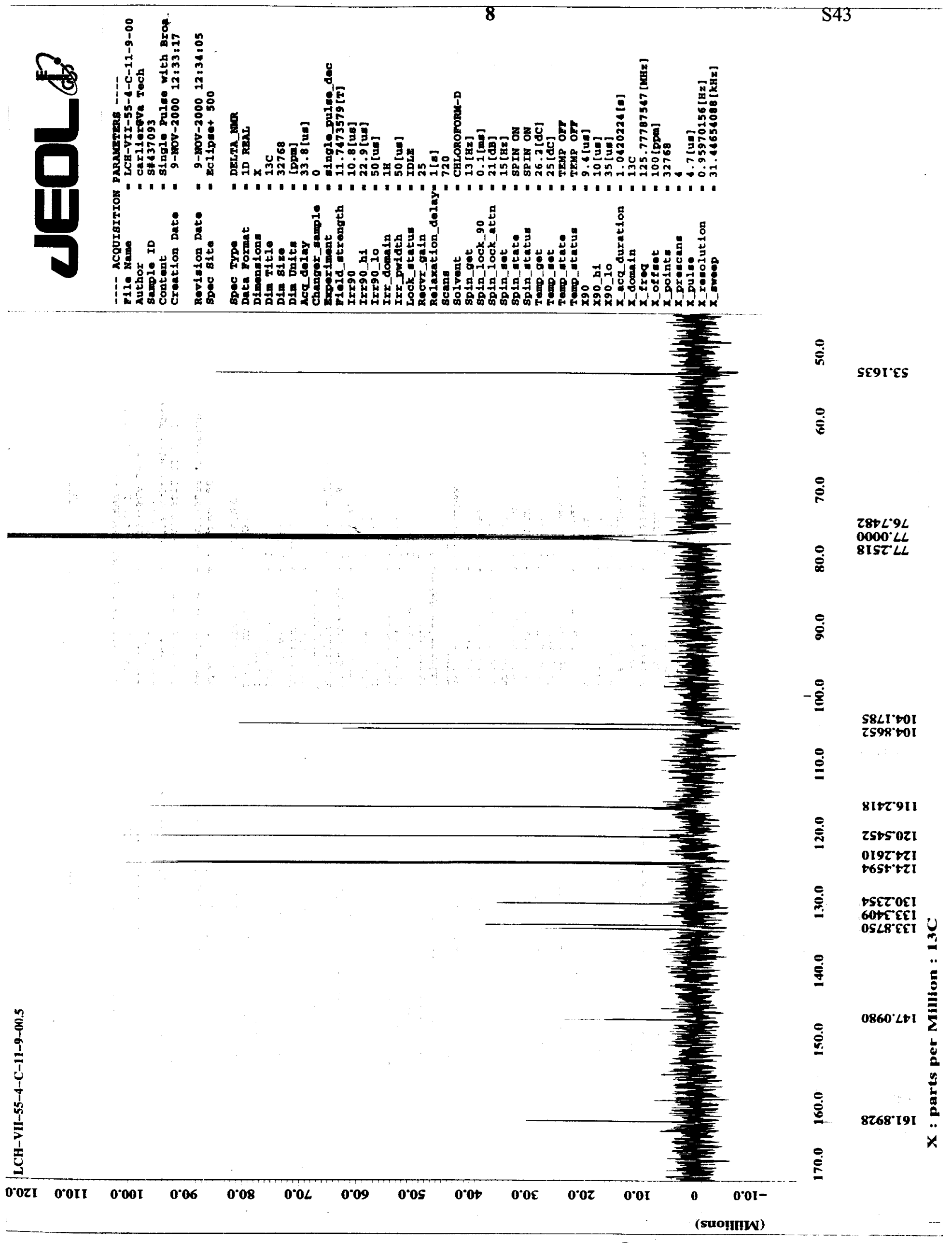



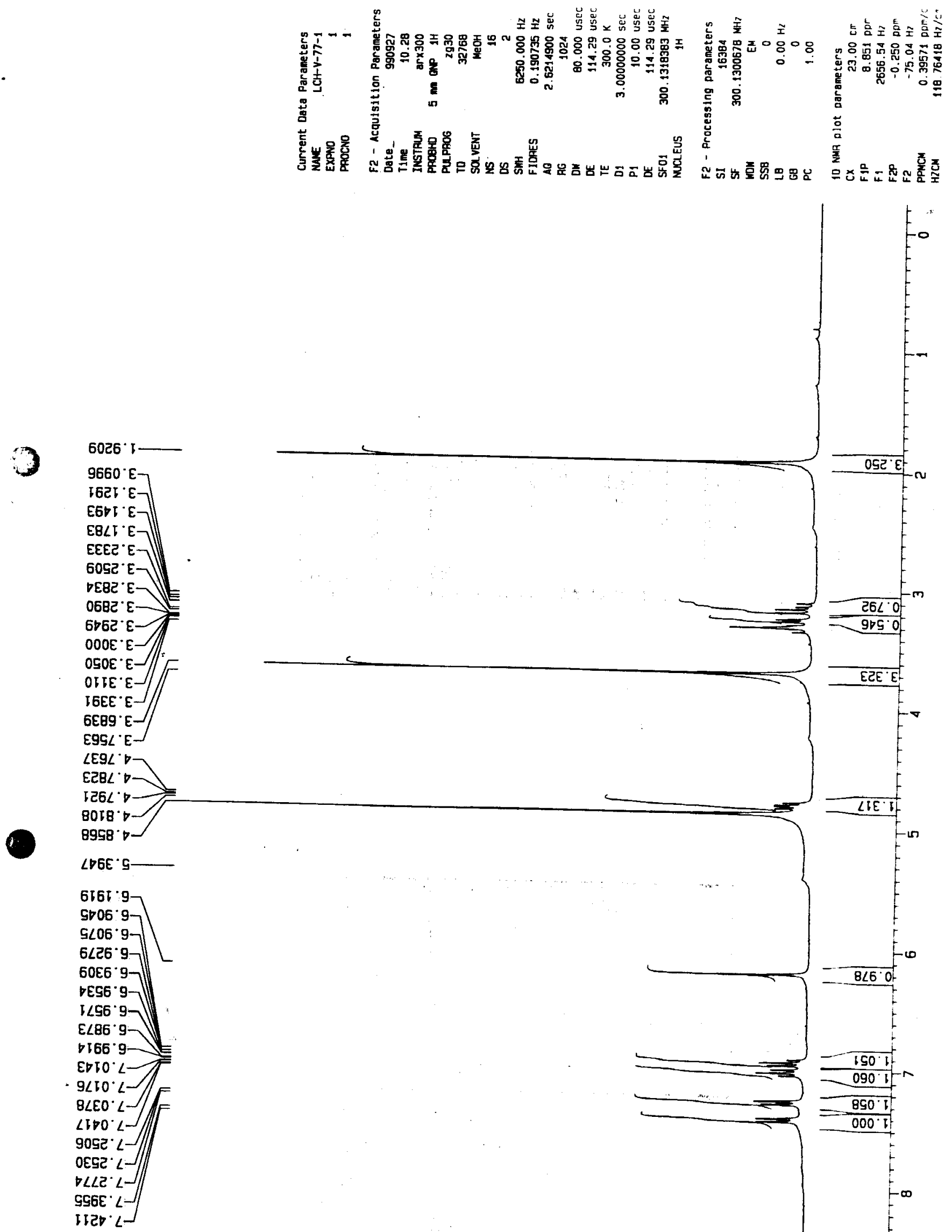

undd

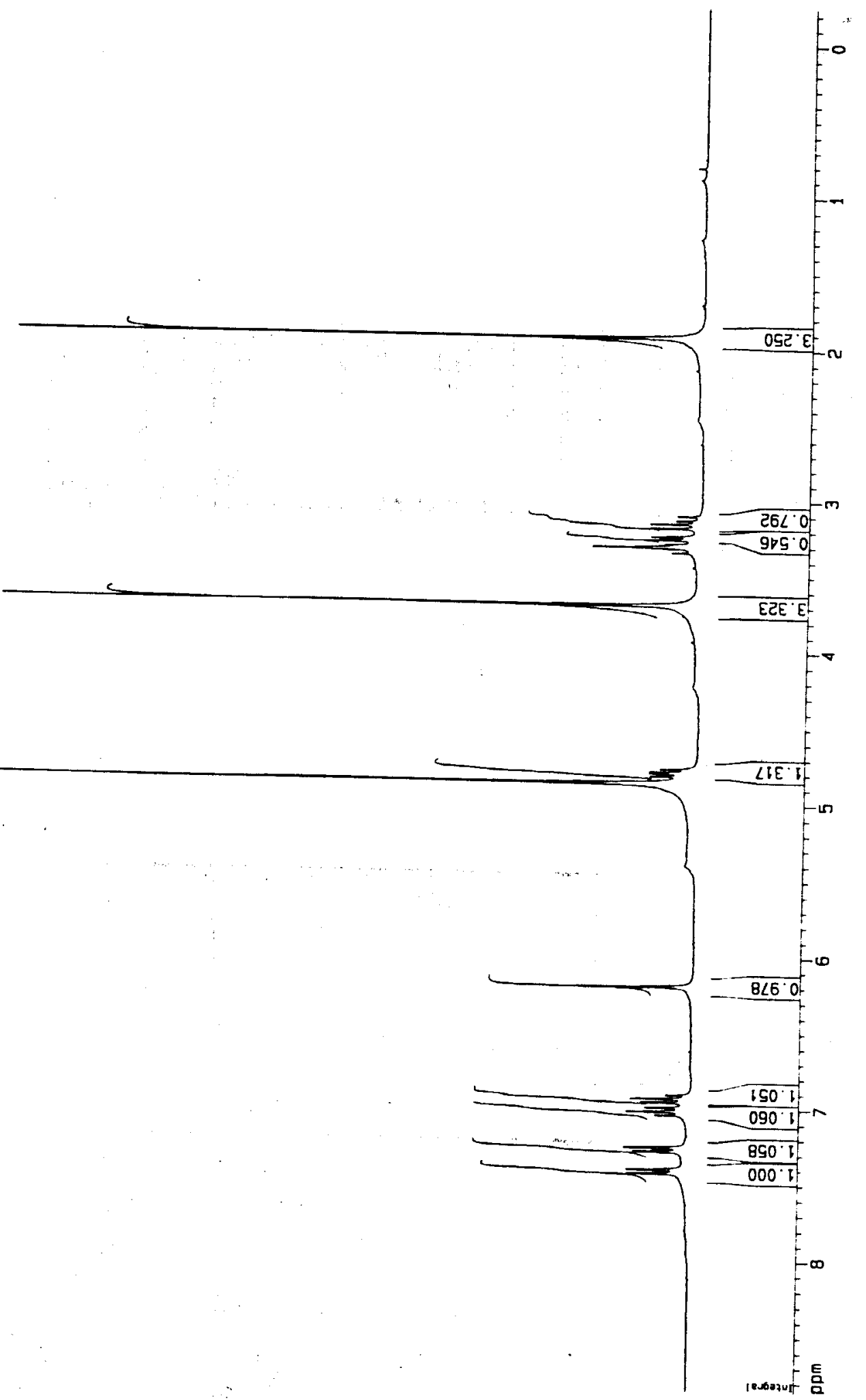




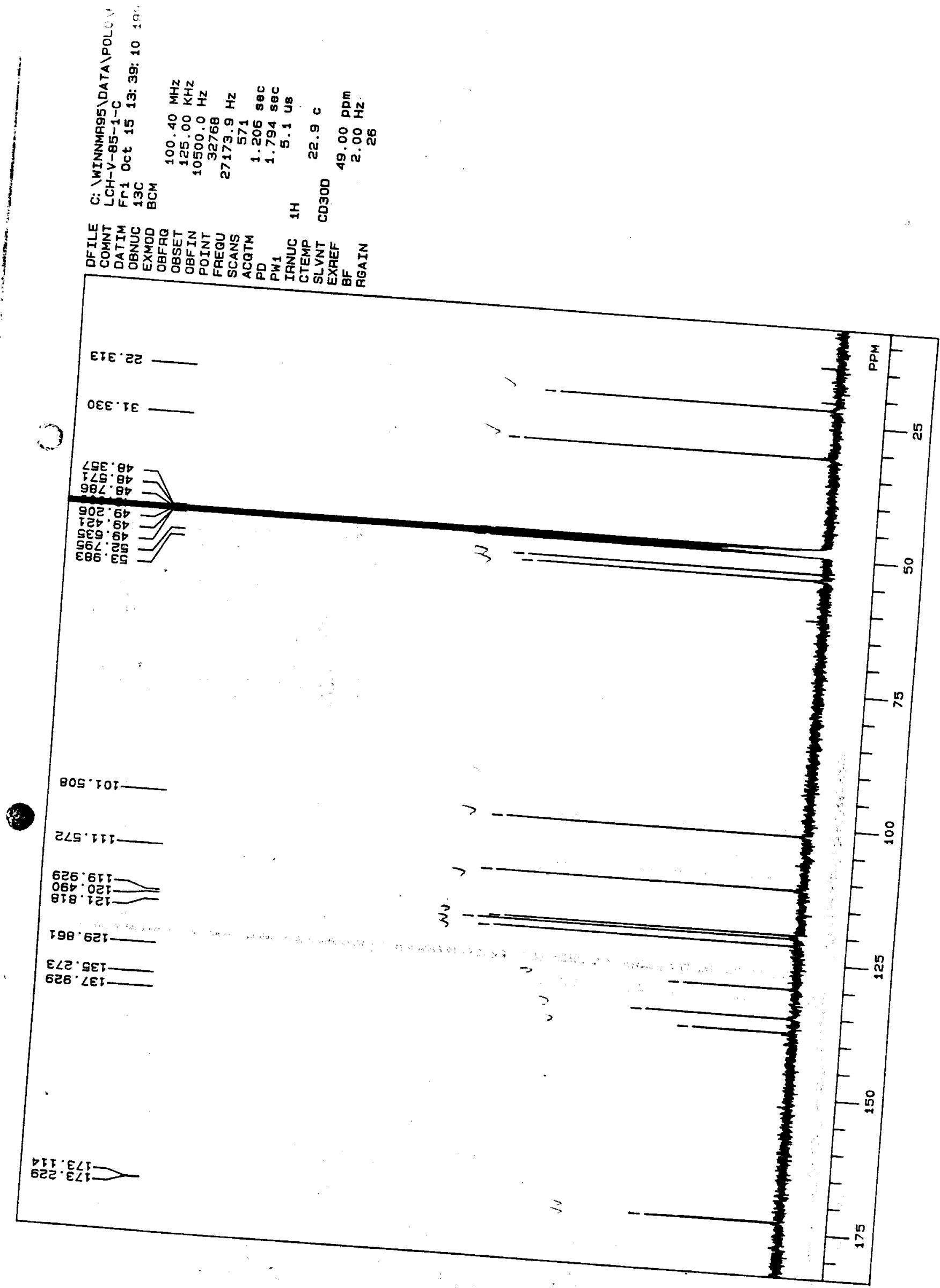



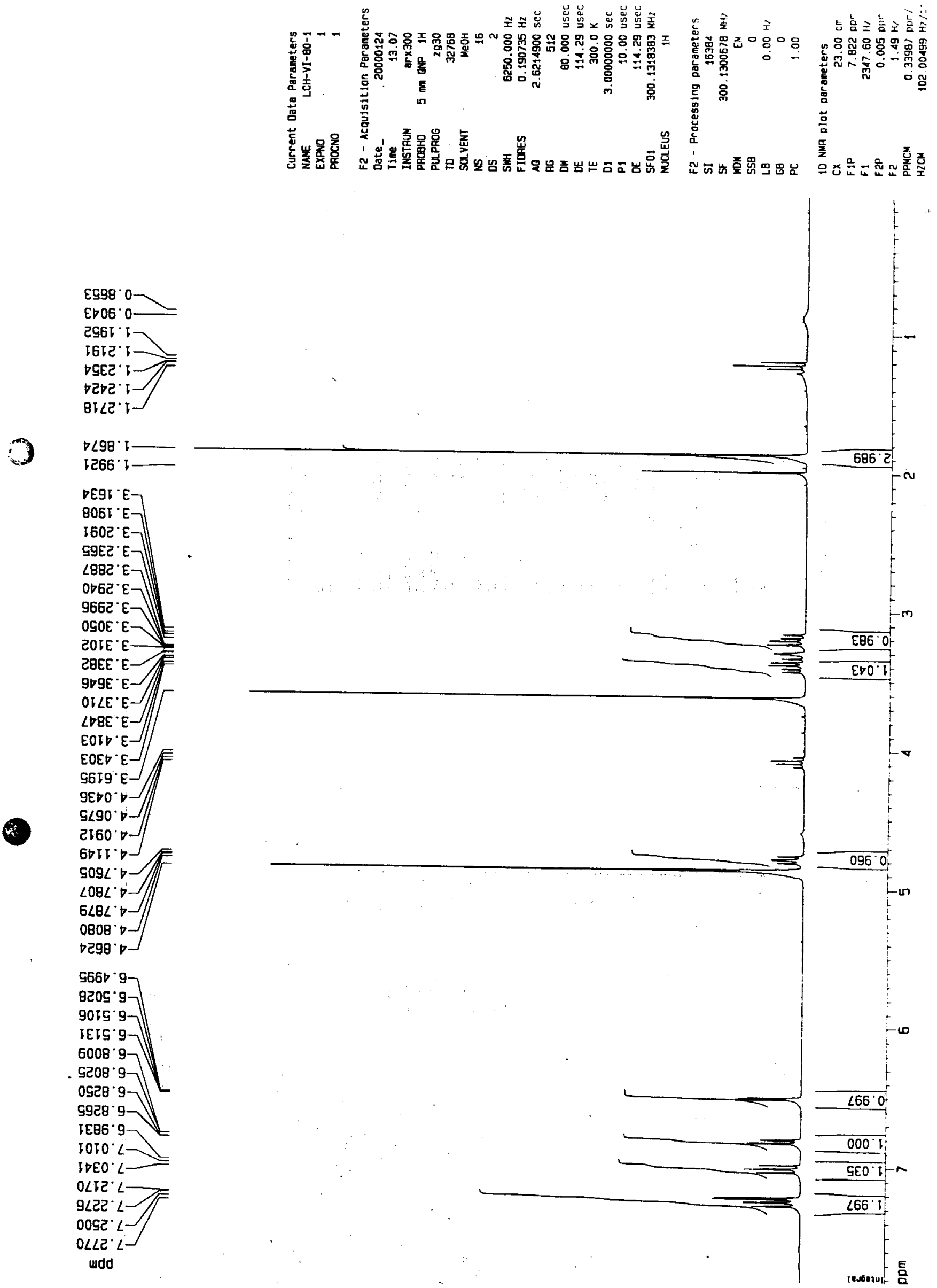

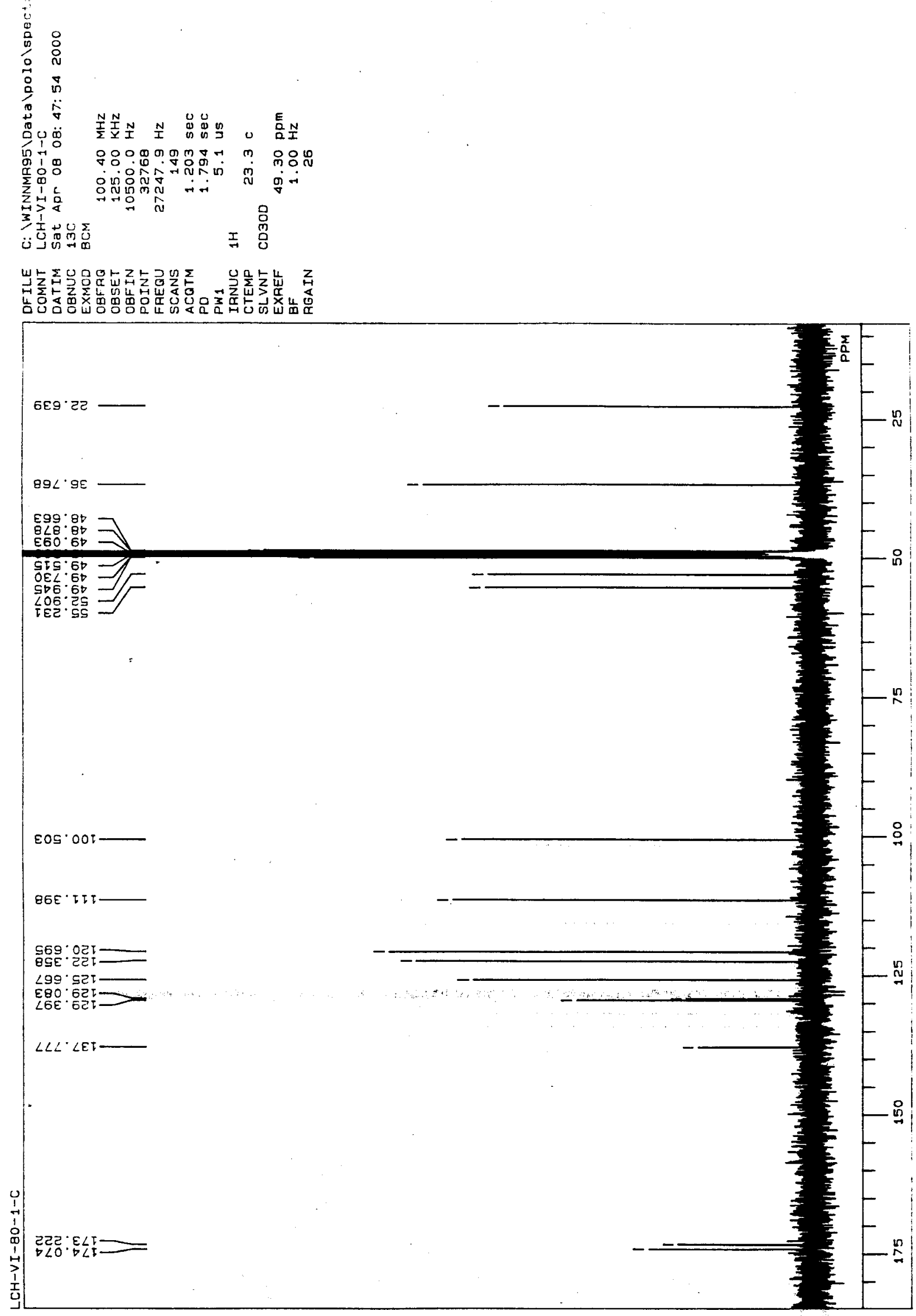


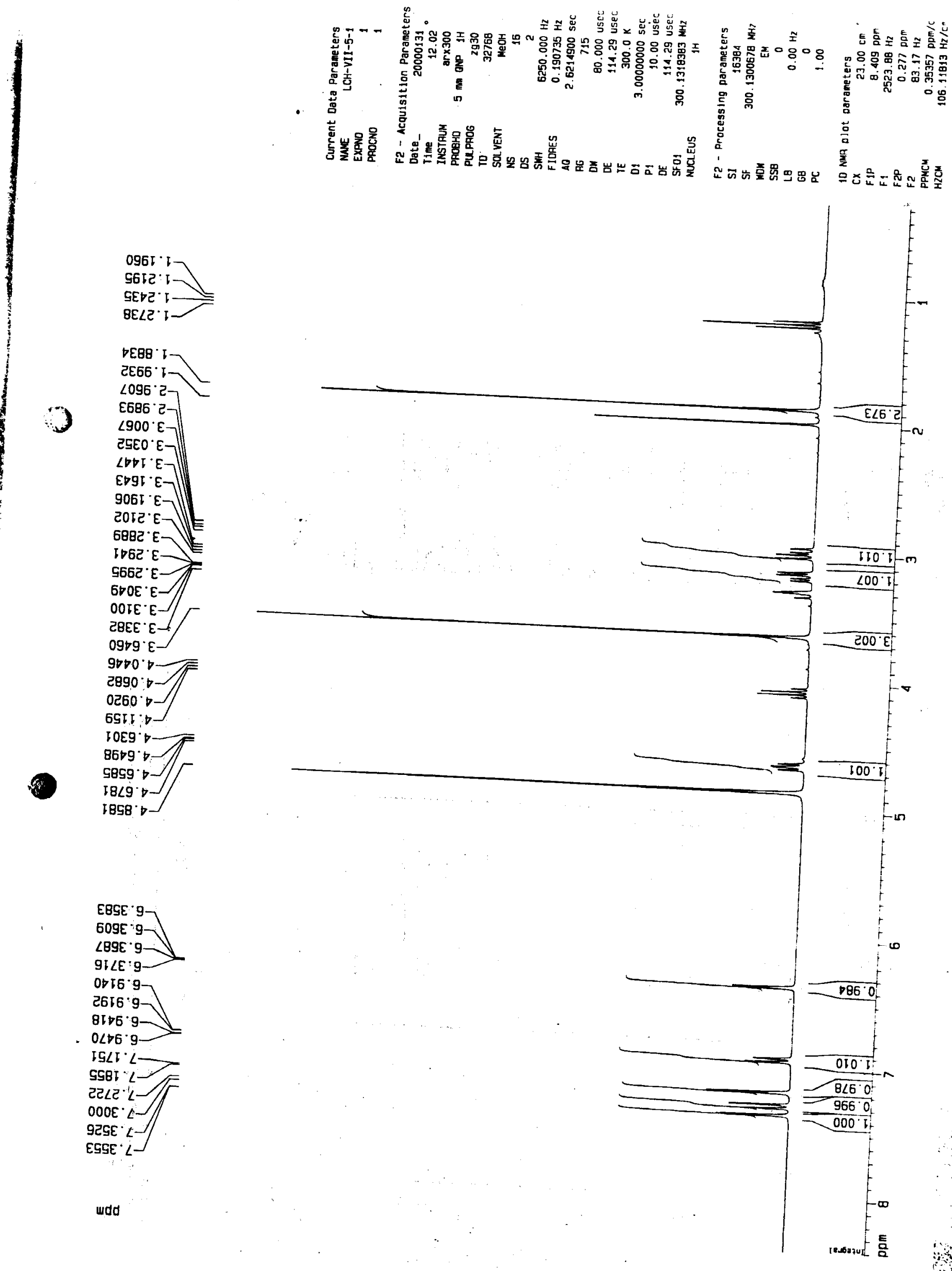




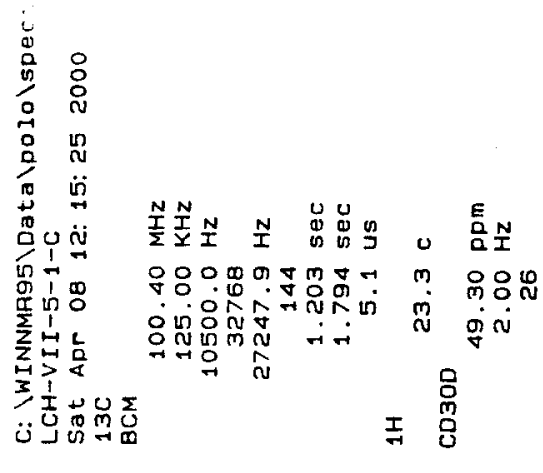

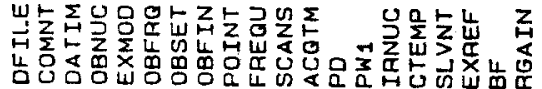

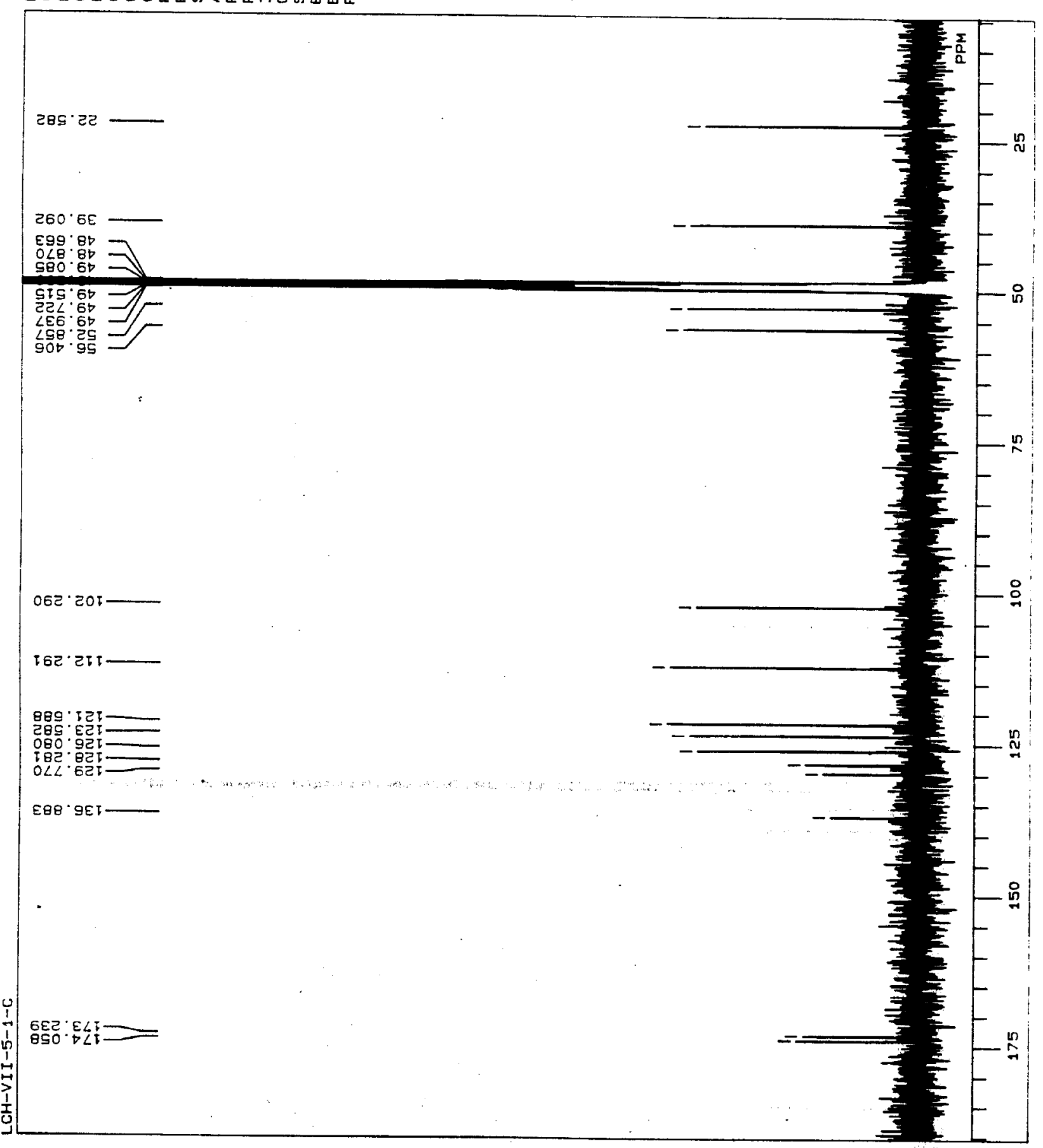



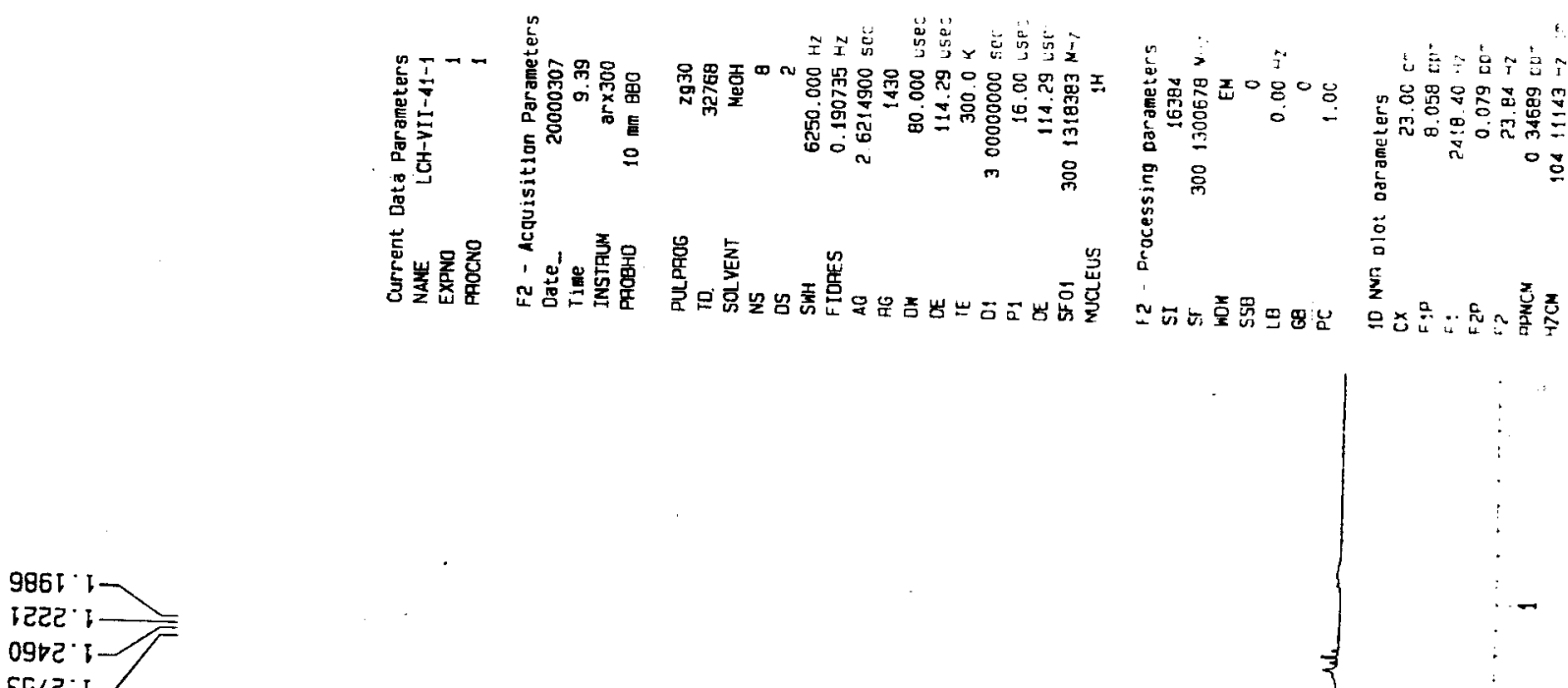

ESLC' 1-

$D 068^{\circ} \mathrm{L}$

2966. I-

$6686^{\circ} \mathrm{C}$

$6 \angle 10^{\circ} E-$

$\angle S E O^{\circ} E$

$8 E 90^{\circ} \varepsilon-$

$\angle I S T^{\circ} E-$

$\triangle\left[B I^{\circ} \varepsilon>\right.$

9LOC $E$

$E \angle Z C^{\circ} E-$

068ट $E$

$\triangle \square \sigma C \cdot E-$

966ट ' $E-$

GDOE' $\varepsilon-$

LOLE.E

$\angle D 59^{\circ} \mathrm{E}-$

$80 \angle 0^{\circ} \circ$

$5060 \%$

c6ro

E699.
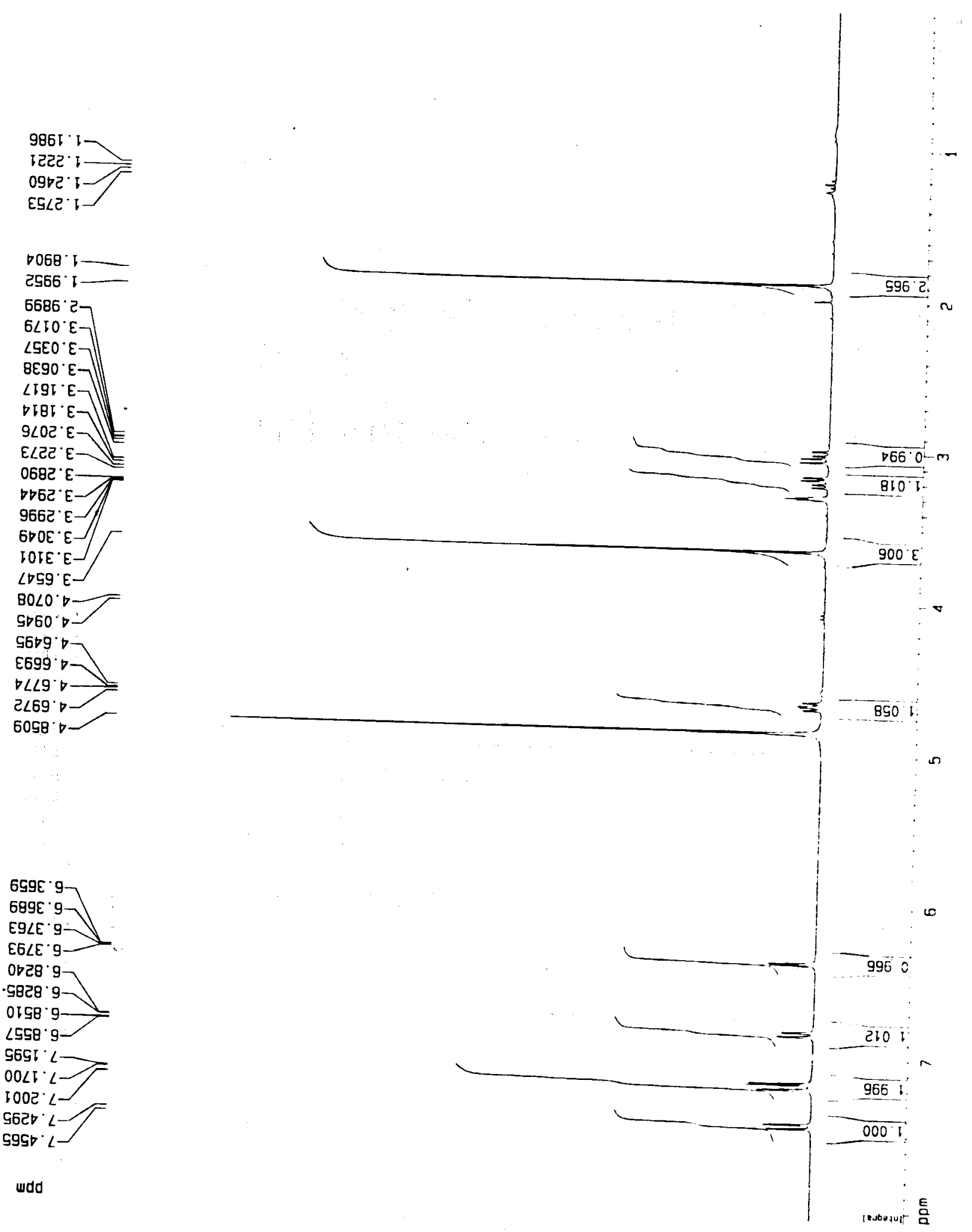

689E' 9

E9LE 9 -

E6LE' 9

$0 B 28 \cdot 9$

-5828. 9

$0158 \cdot 9$

$\angle 550^{\circ} 9-$

SES ' $\angle$

$00 \angle 1<$

$1002 \angle$

G6टt $\angle$

S9St $L$ 
11d

S51
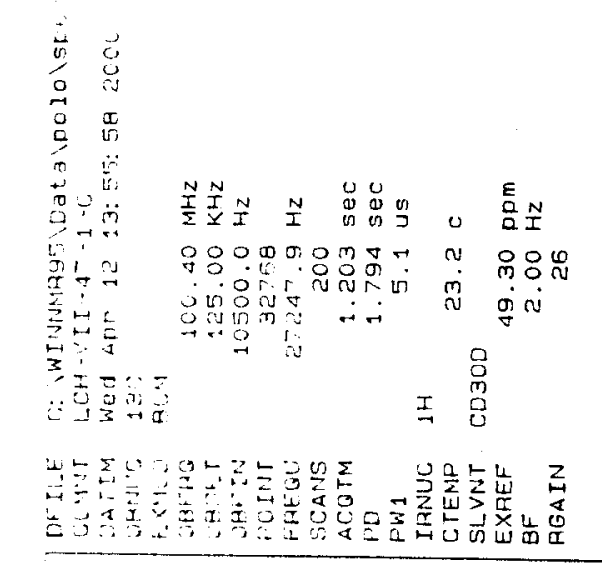

$\operatorname{seg}=$
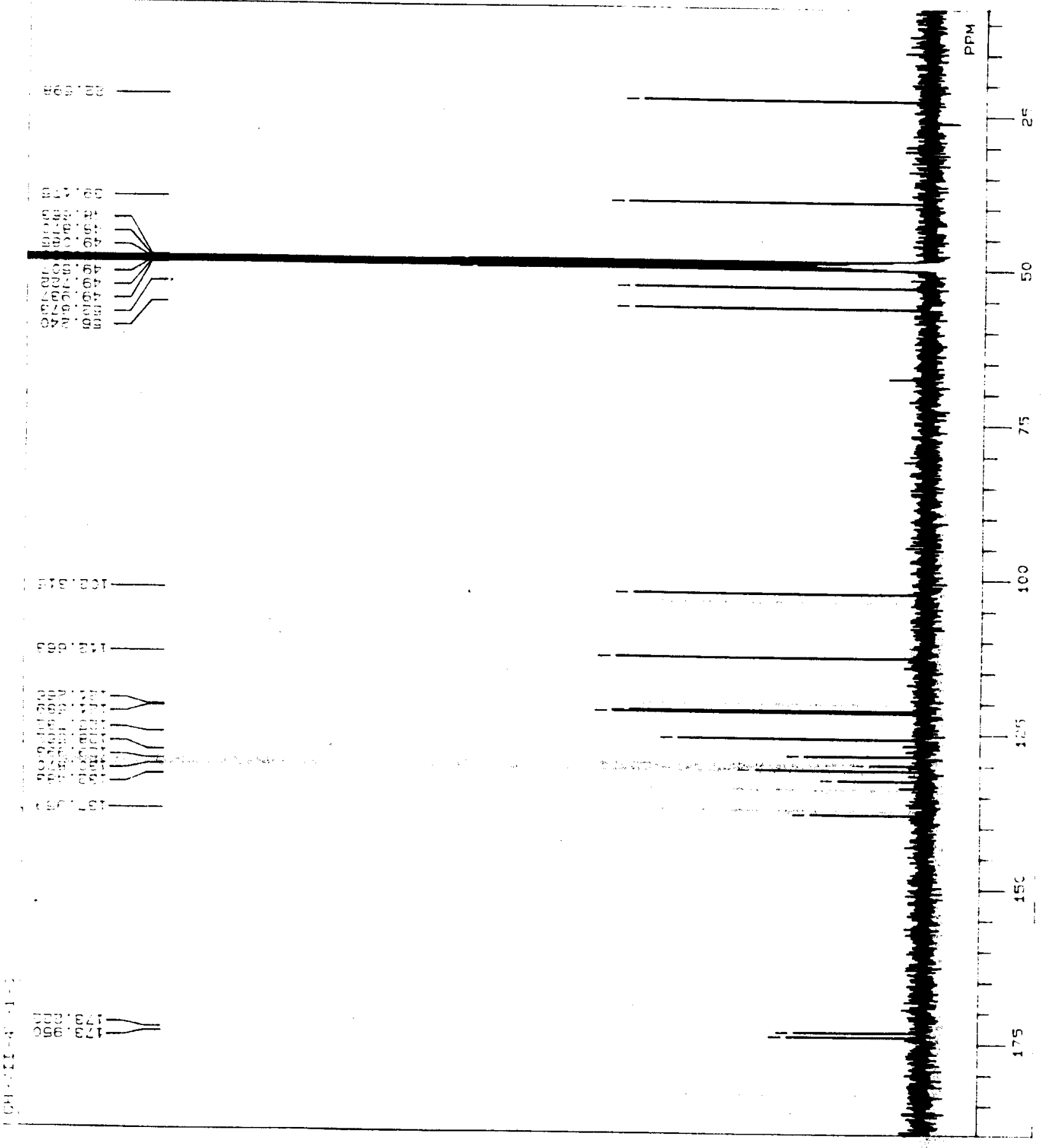


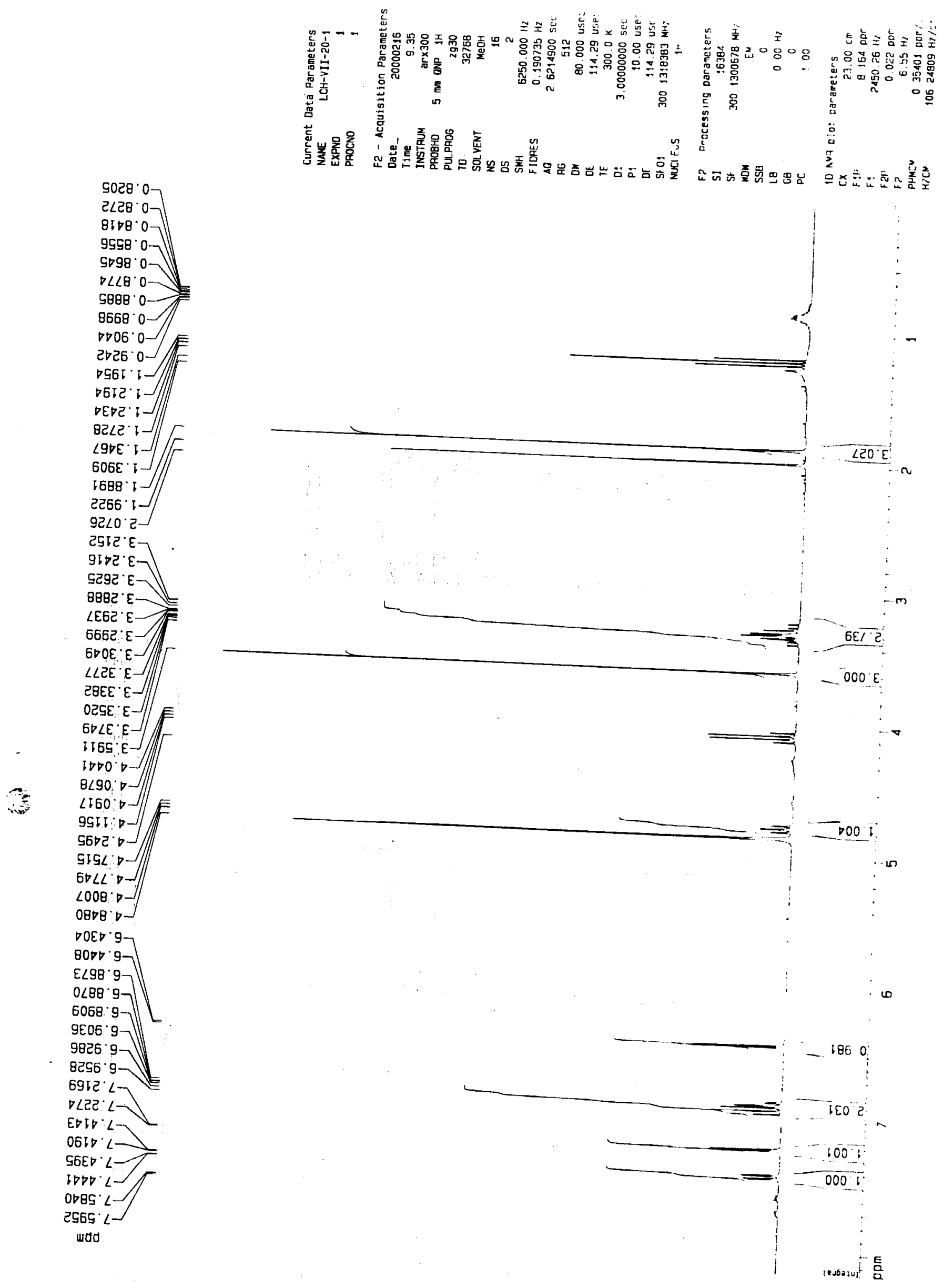


$11 \mathrm{e}$

S53

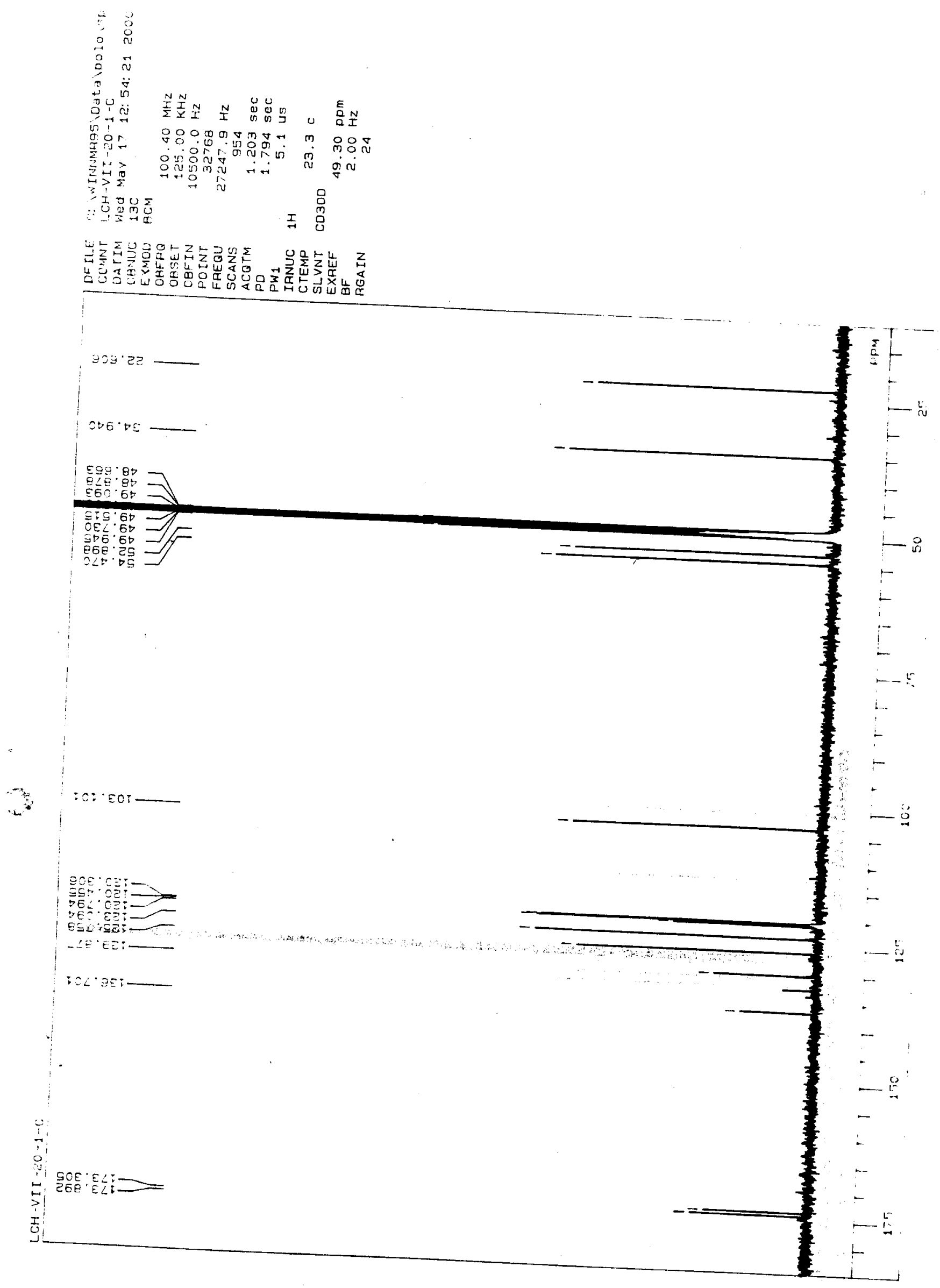



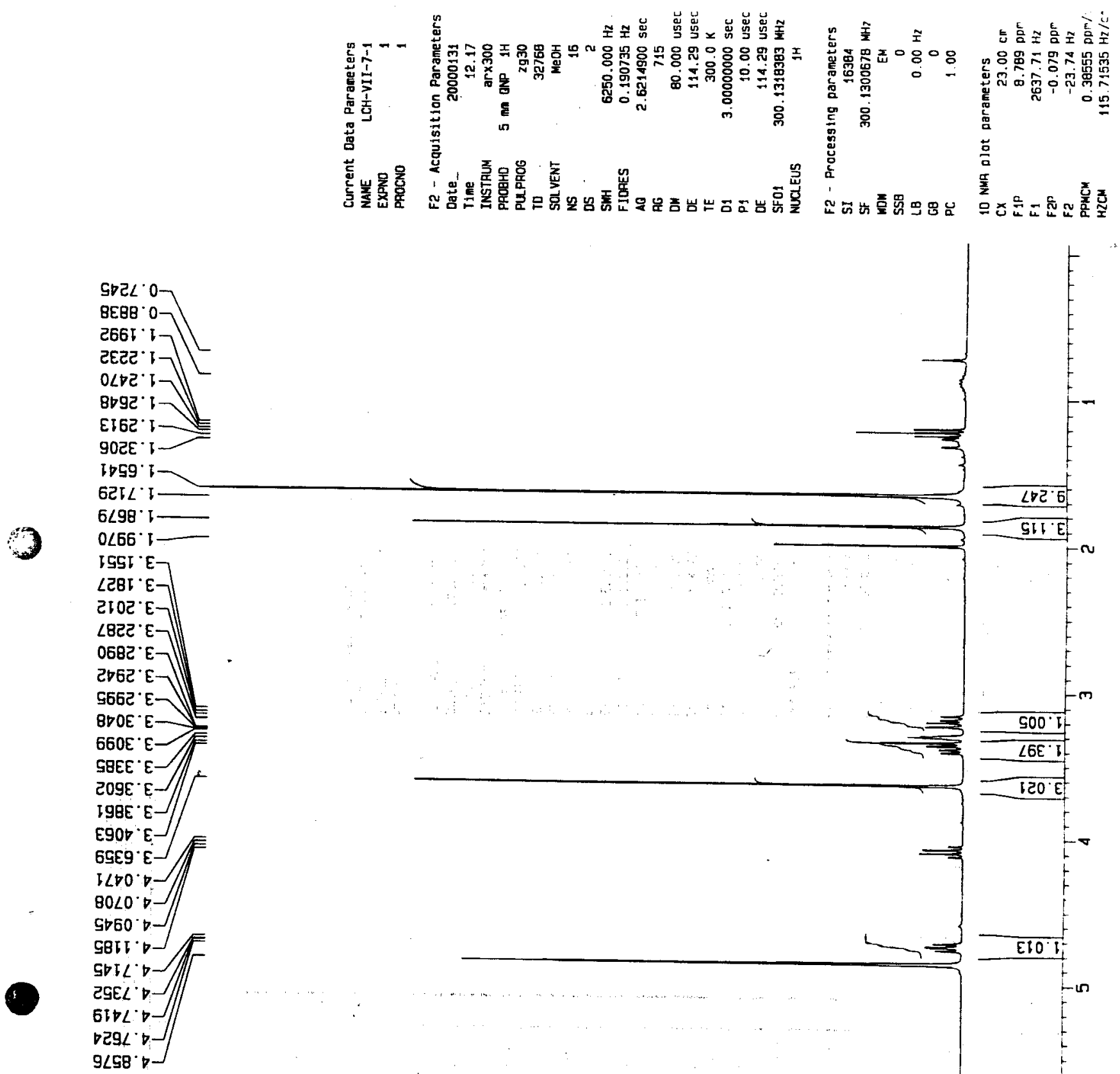

$9 \angle 89 \cdot 9$

$100<\cdot 9$

$5600 . \mathrm{L}$

EVEO $\angle \triangle$

'OLLV'

टDOZ' $\angle$

S6ट2 $L$

EEIS: $\angle$

$0929^{\circ} \angle$

$5666 . \mathrm{L}$

6920.8

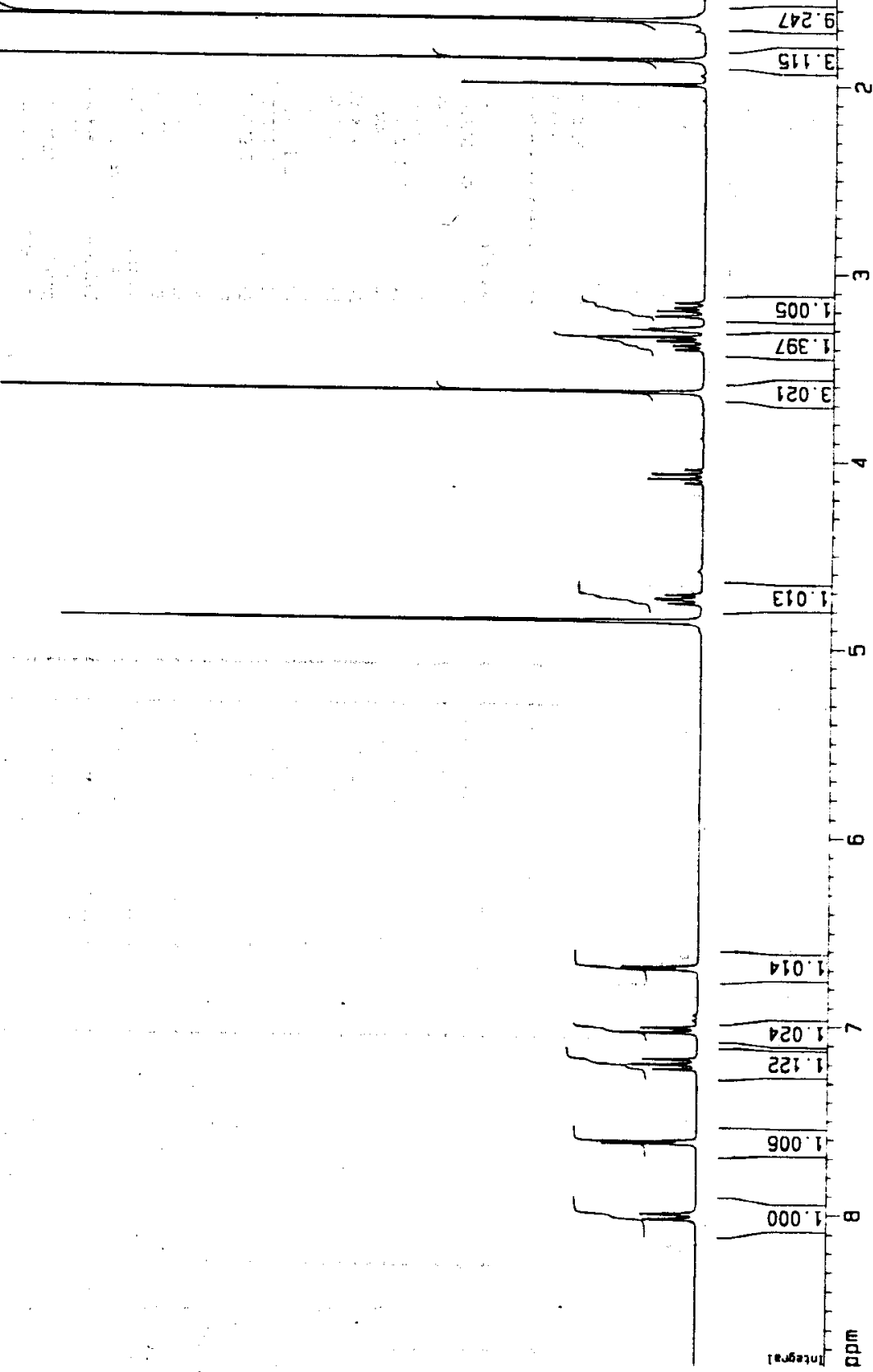




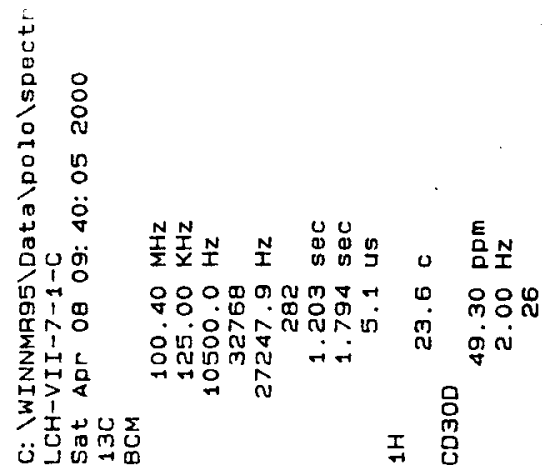

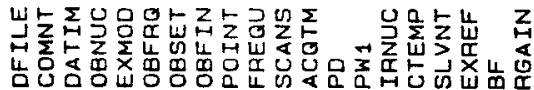

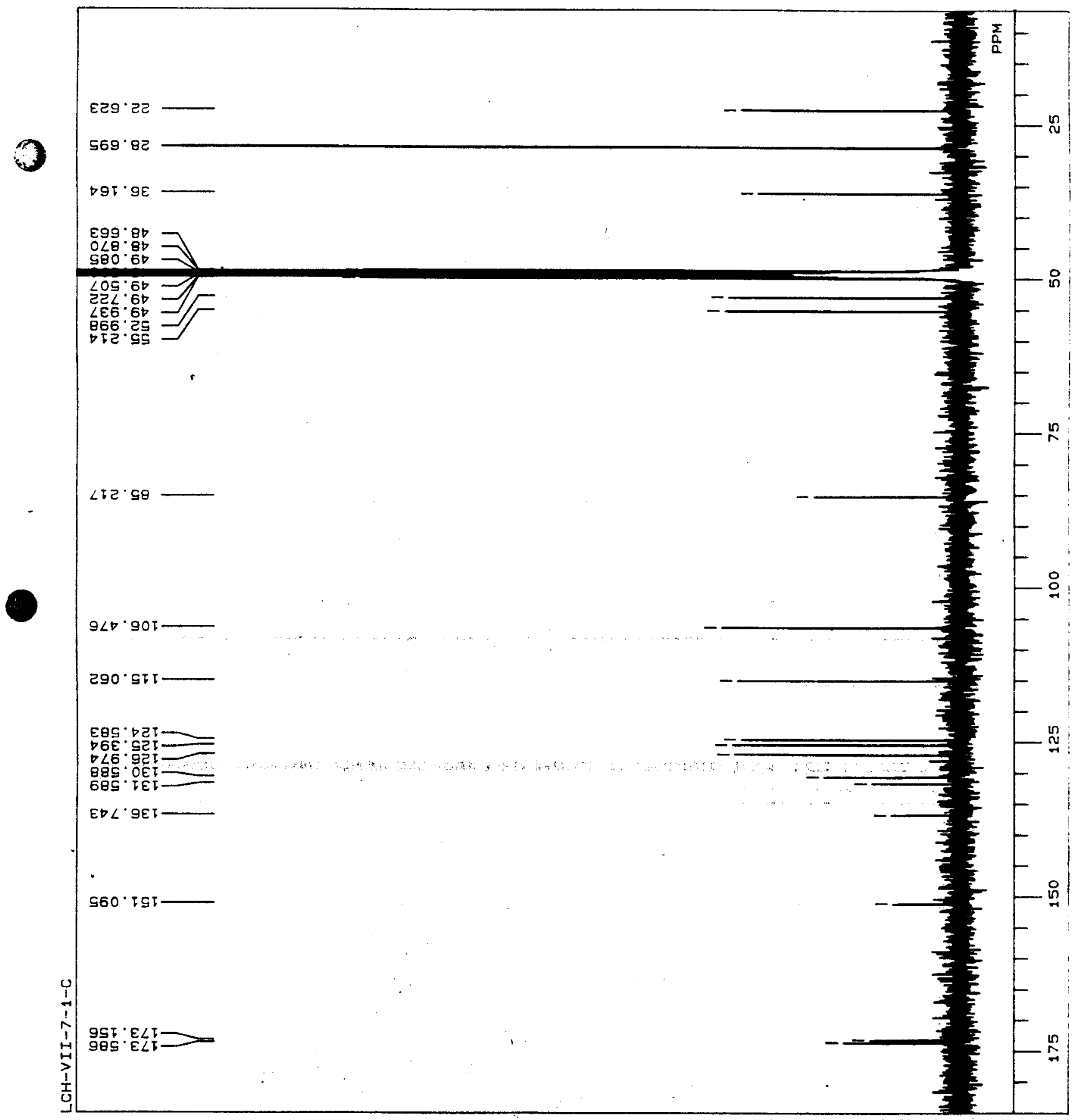




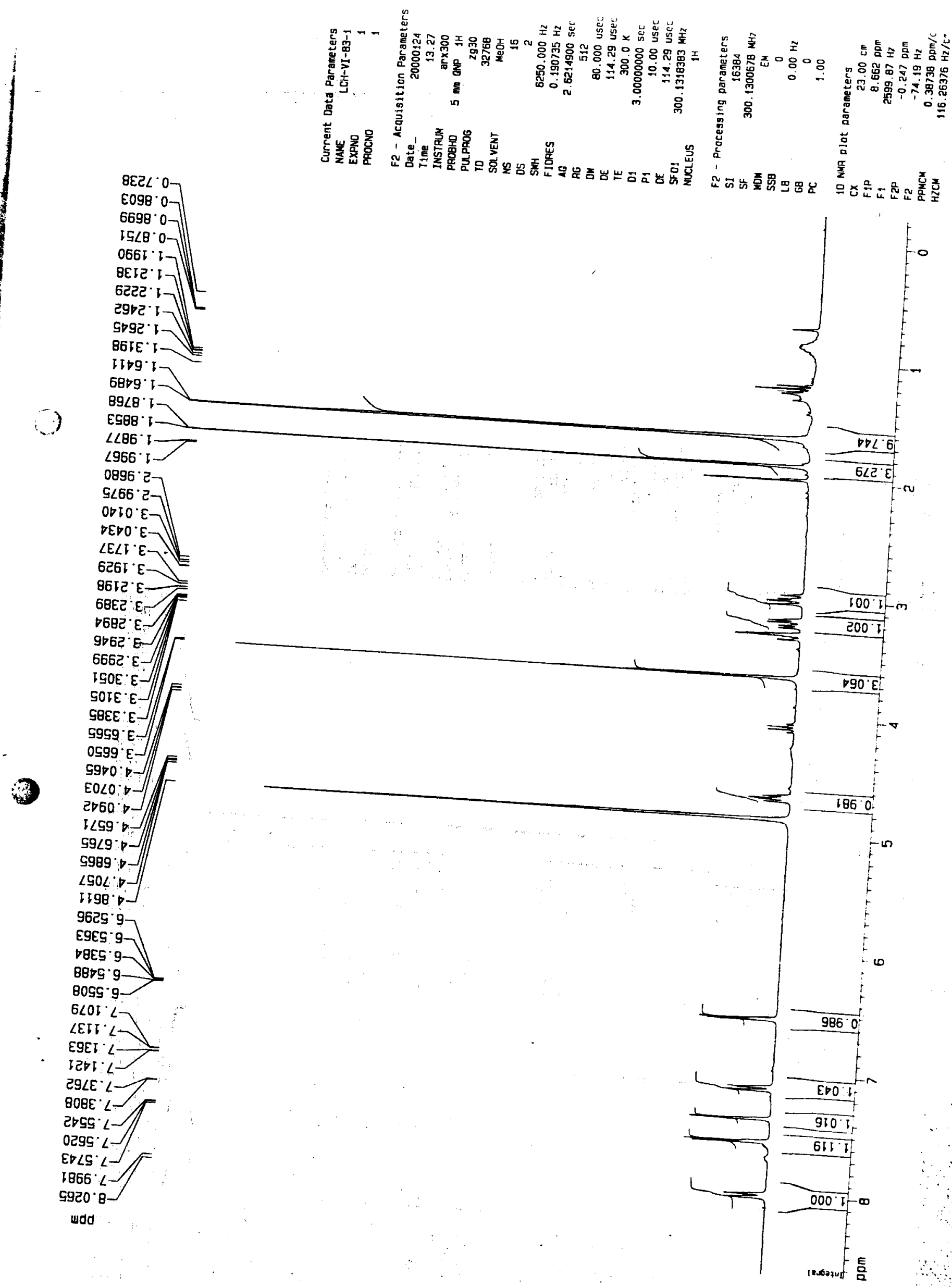




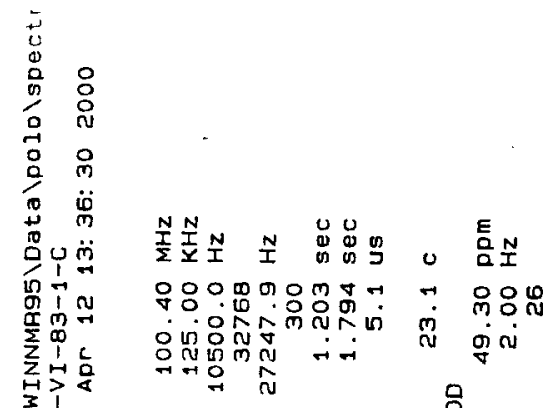

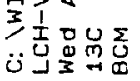

I $\stackrel{\stackrel{\circ}{0}}{0}$

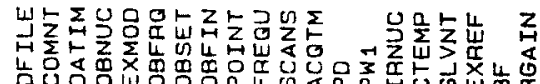

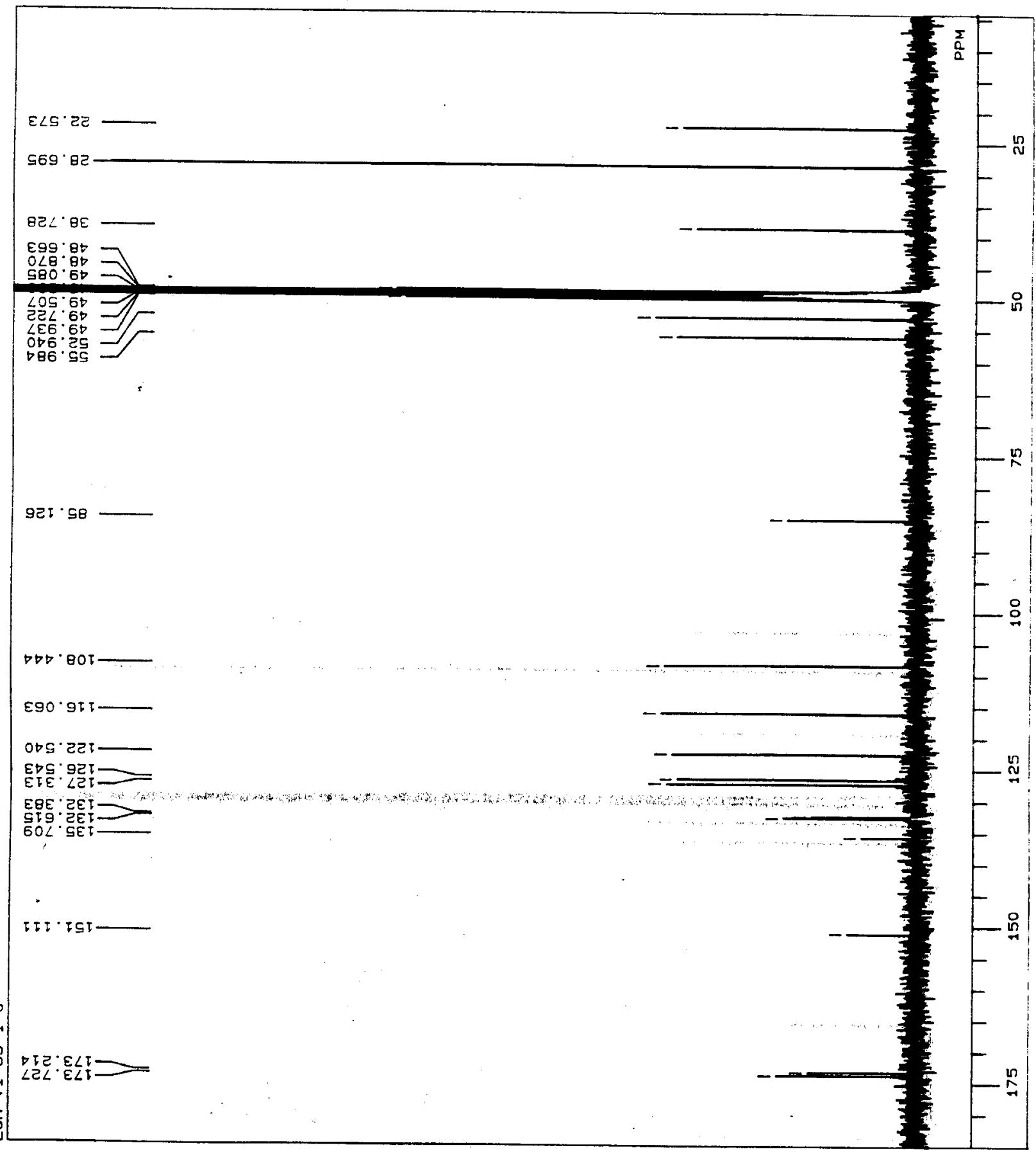

Ot:

E⿱乛龰:92]

EgE:टE

SOL. SET 

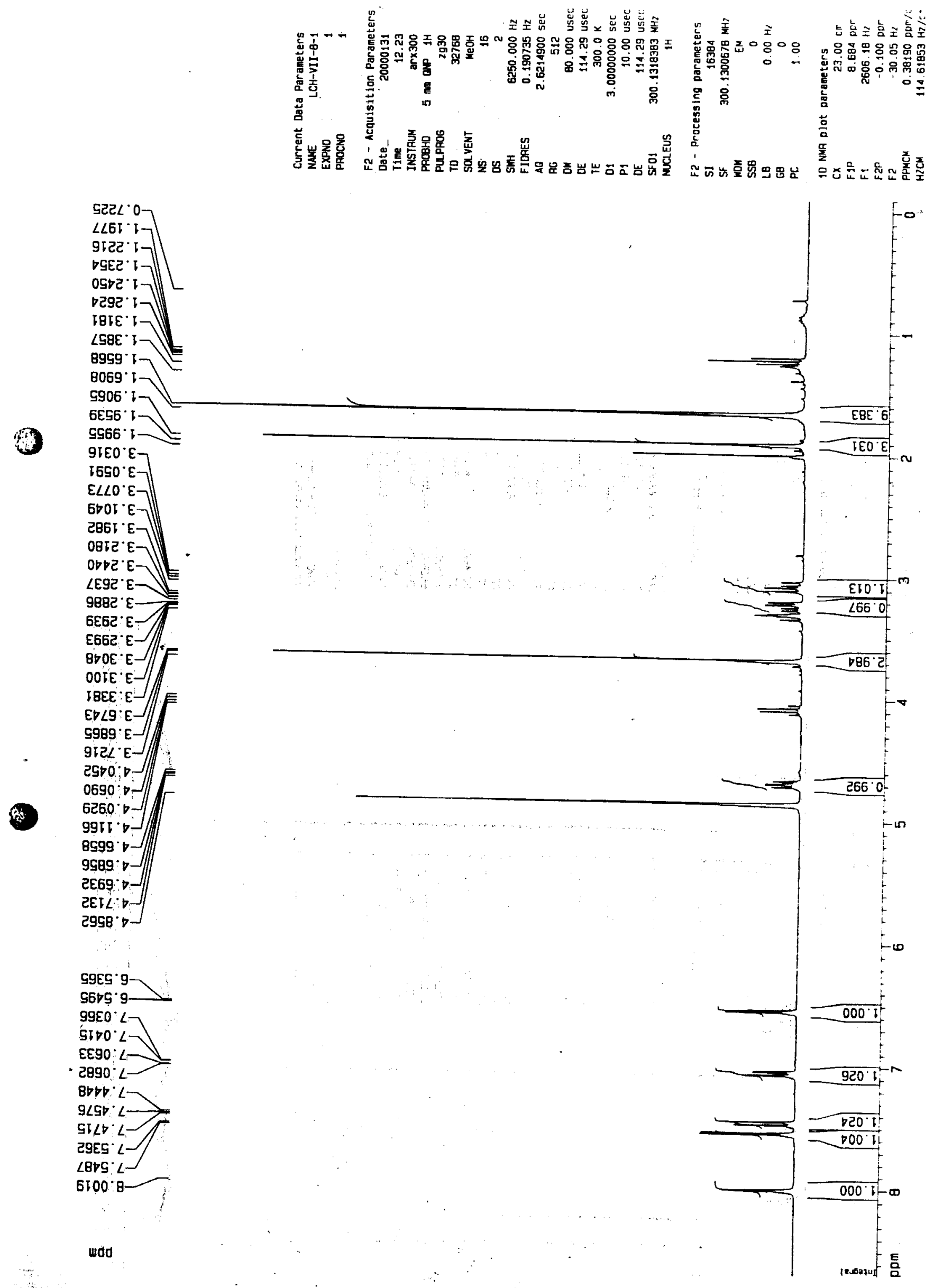

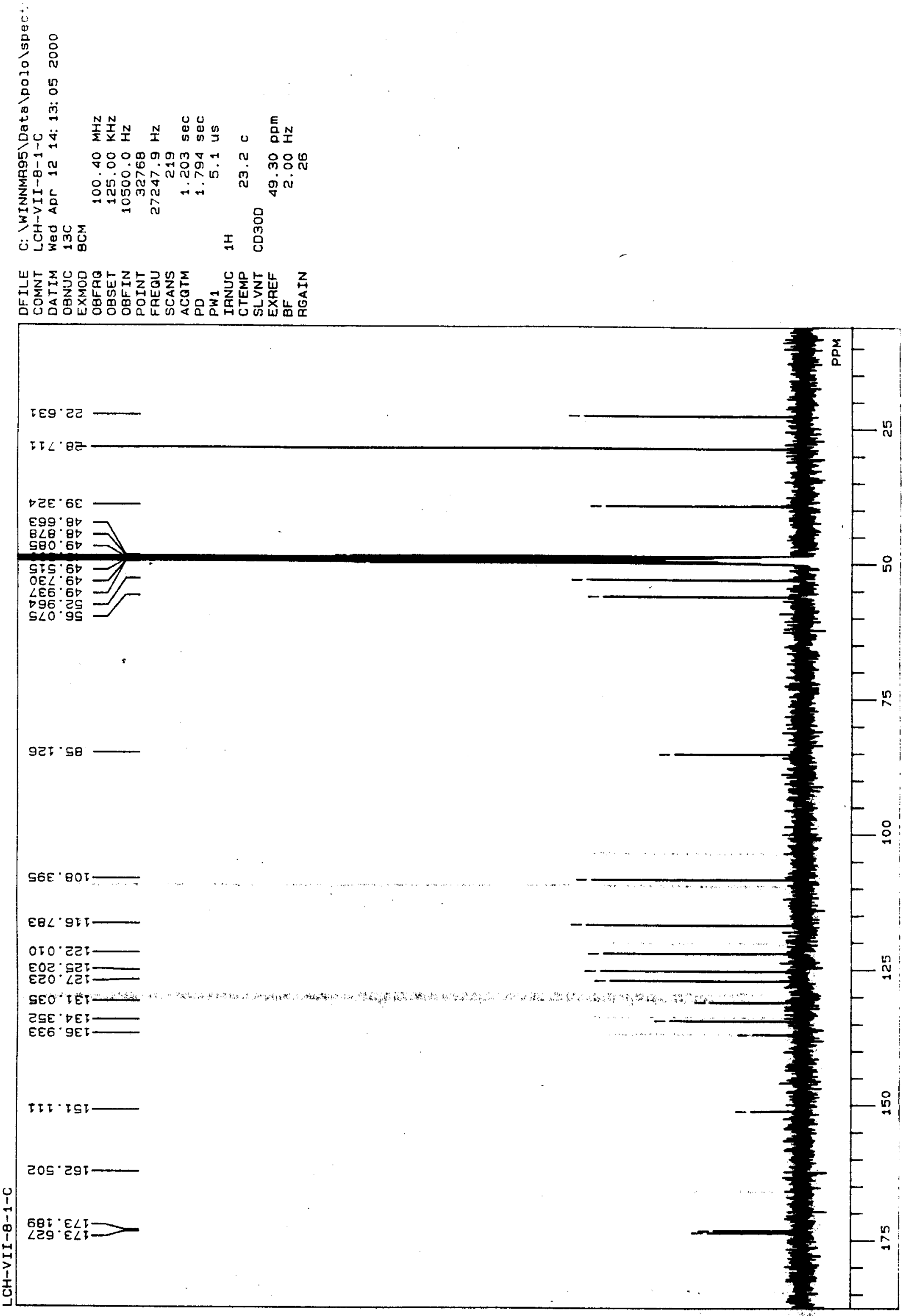

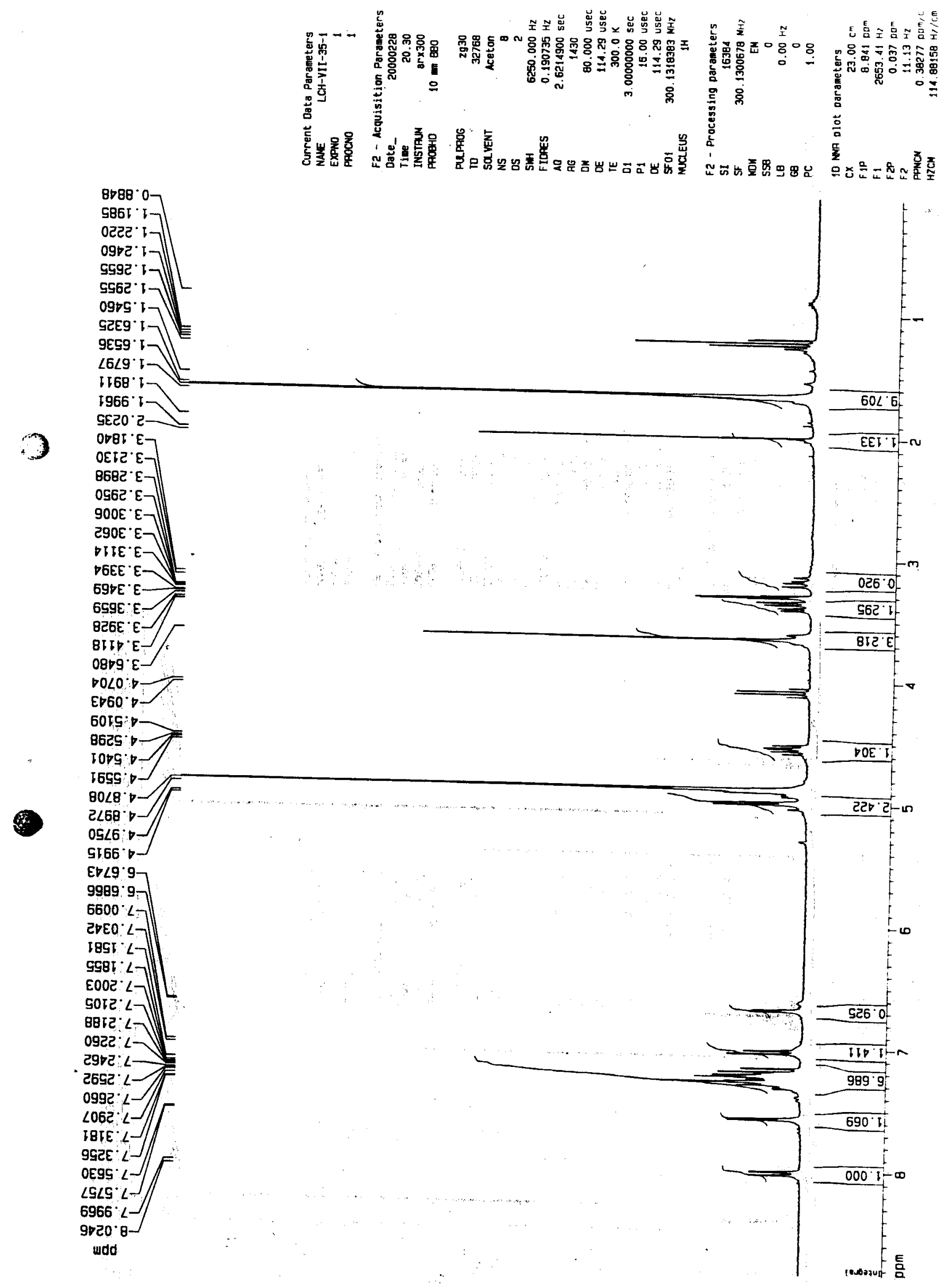


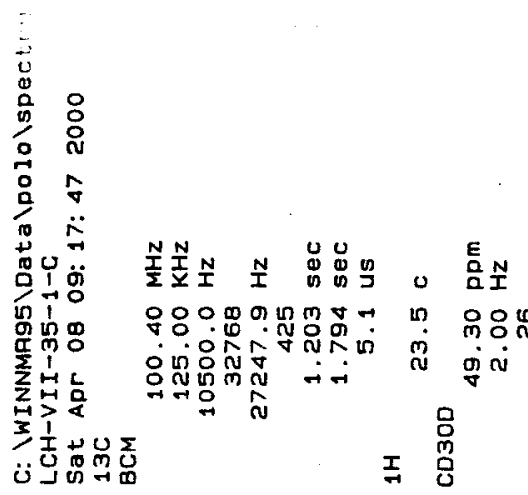

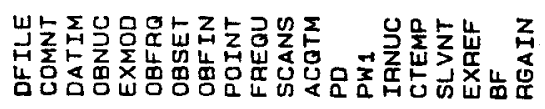

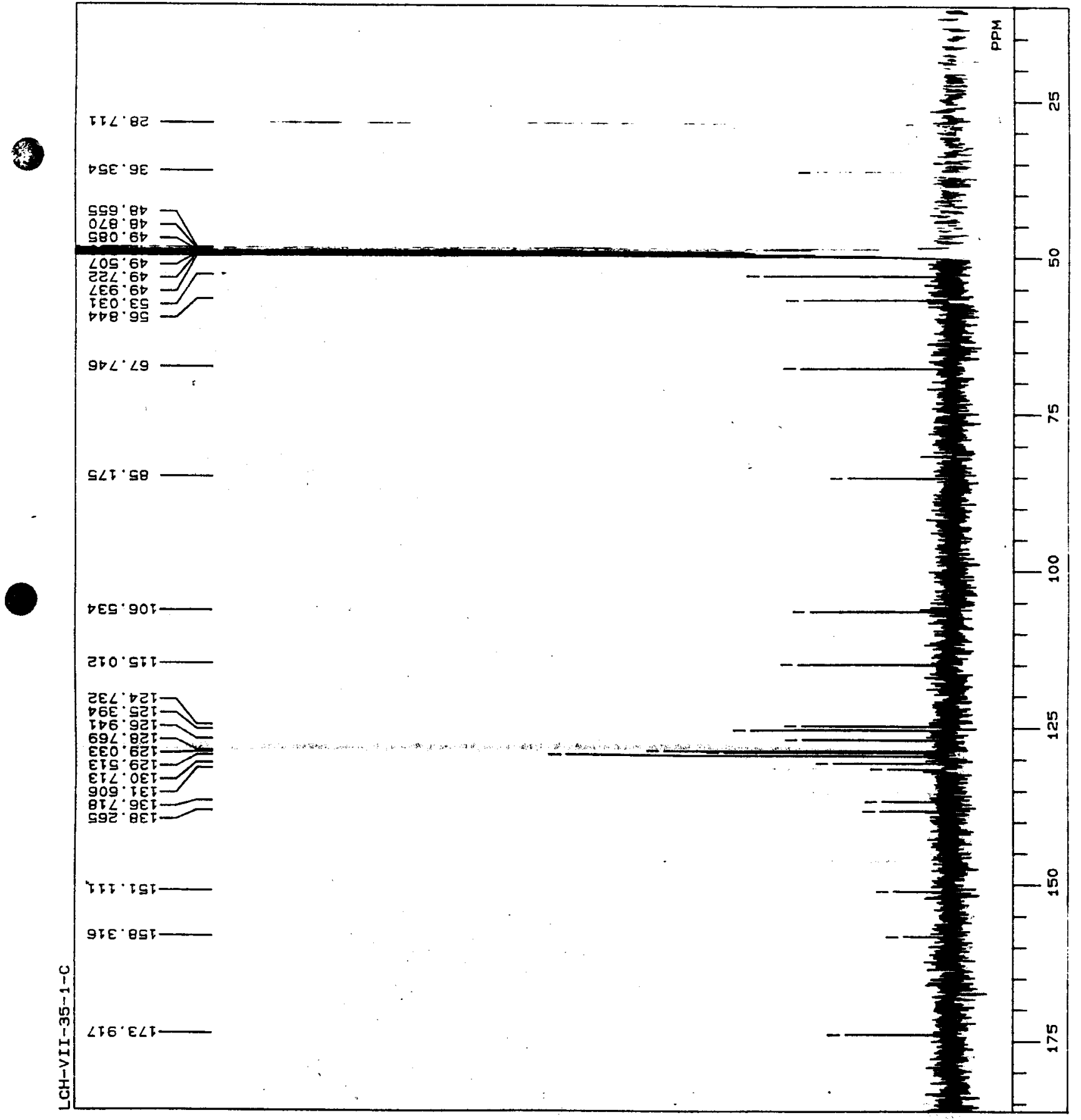



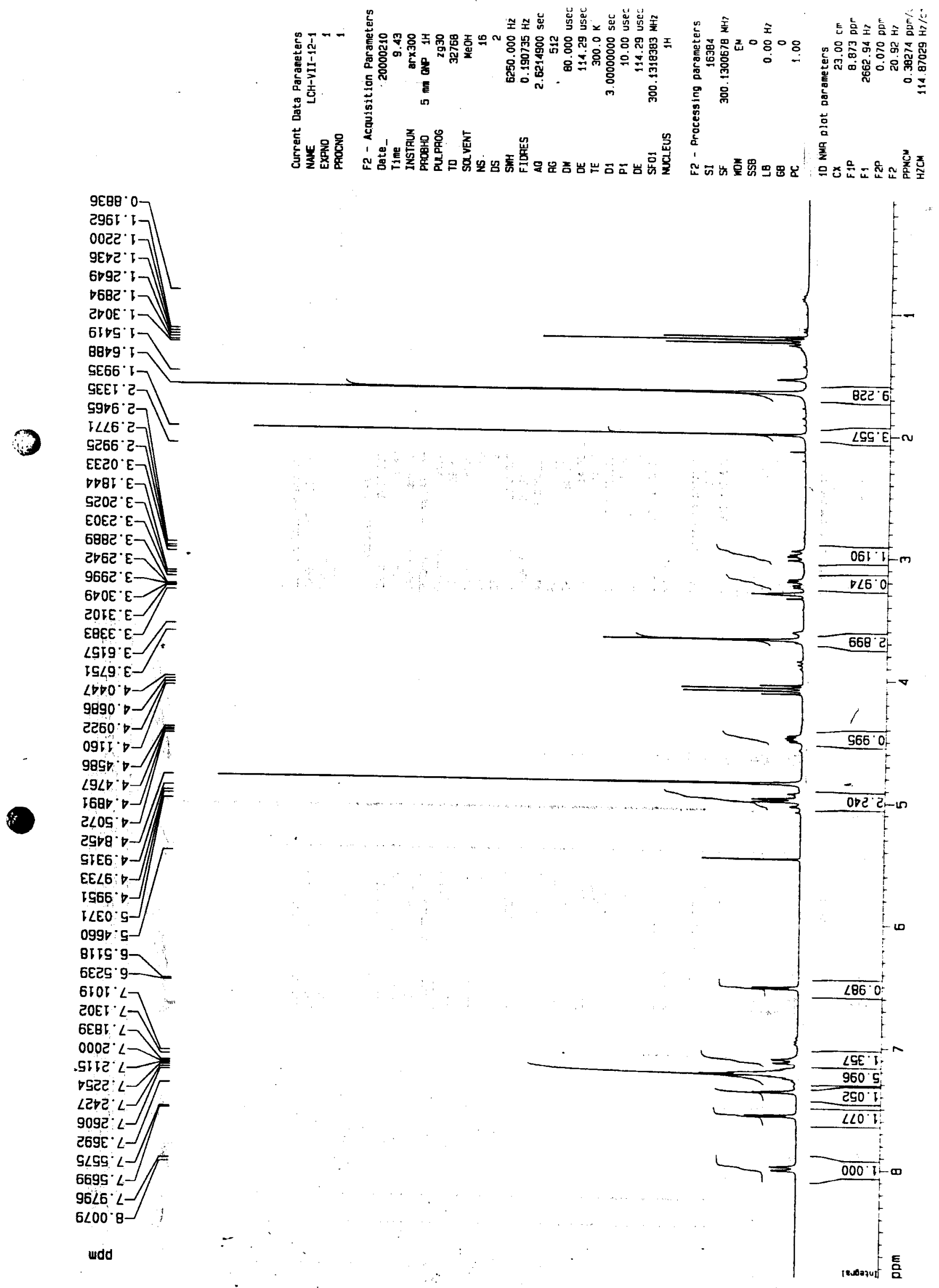


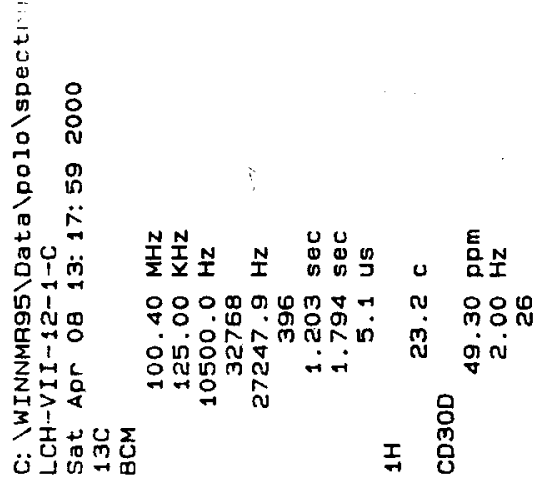

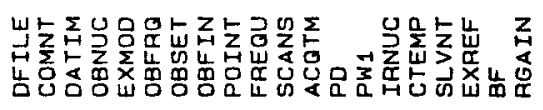

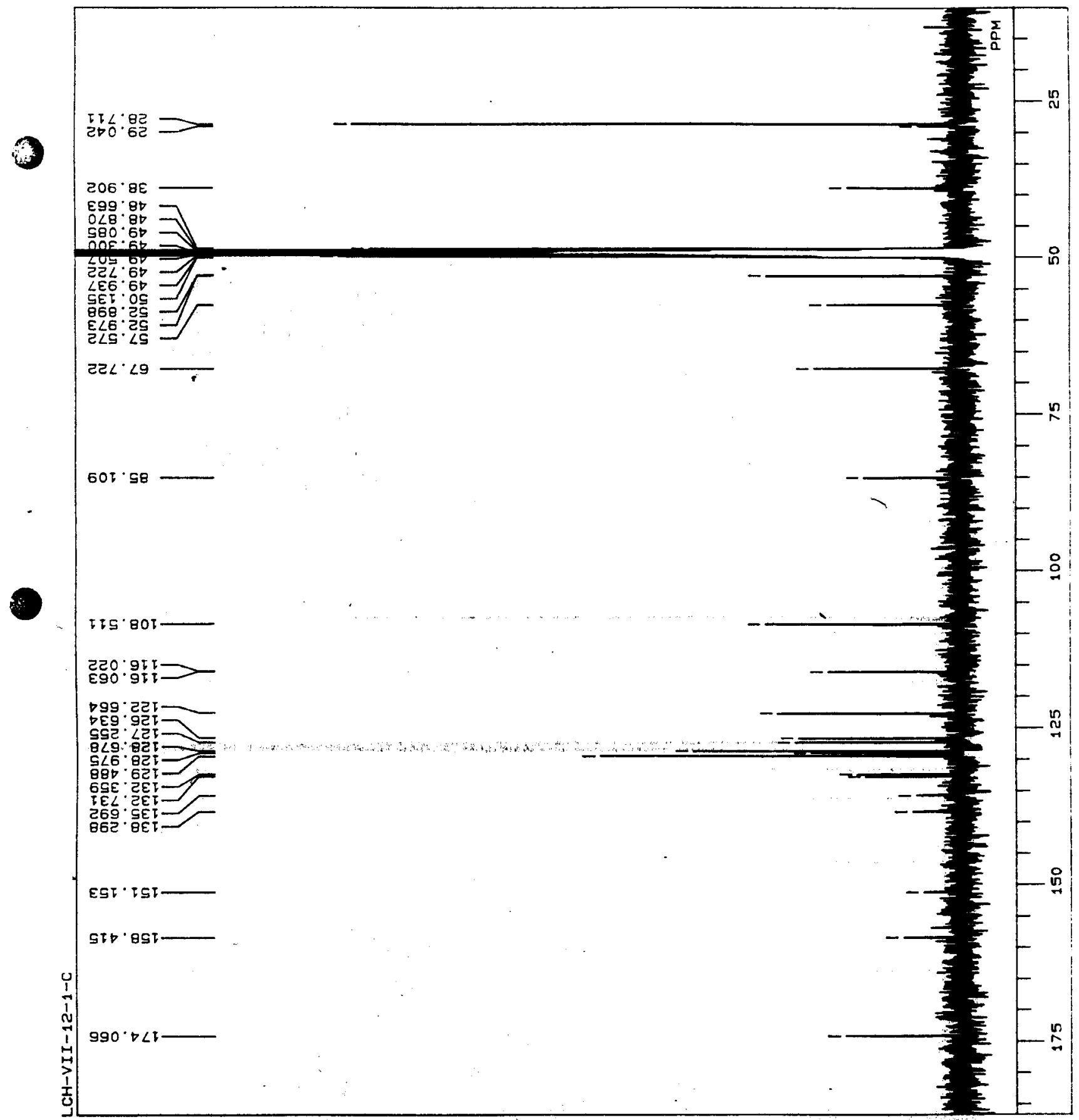



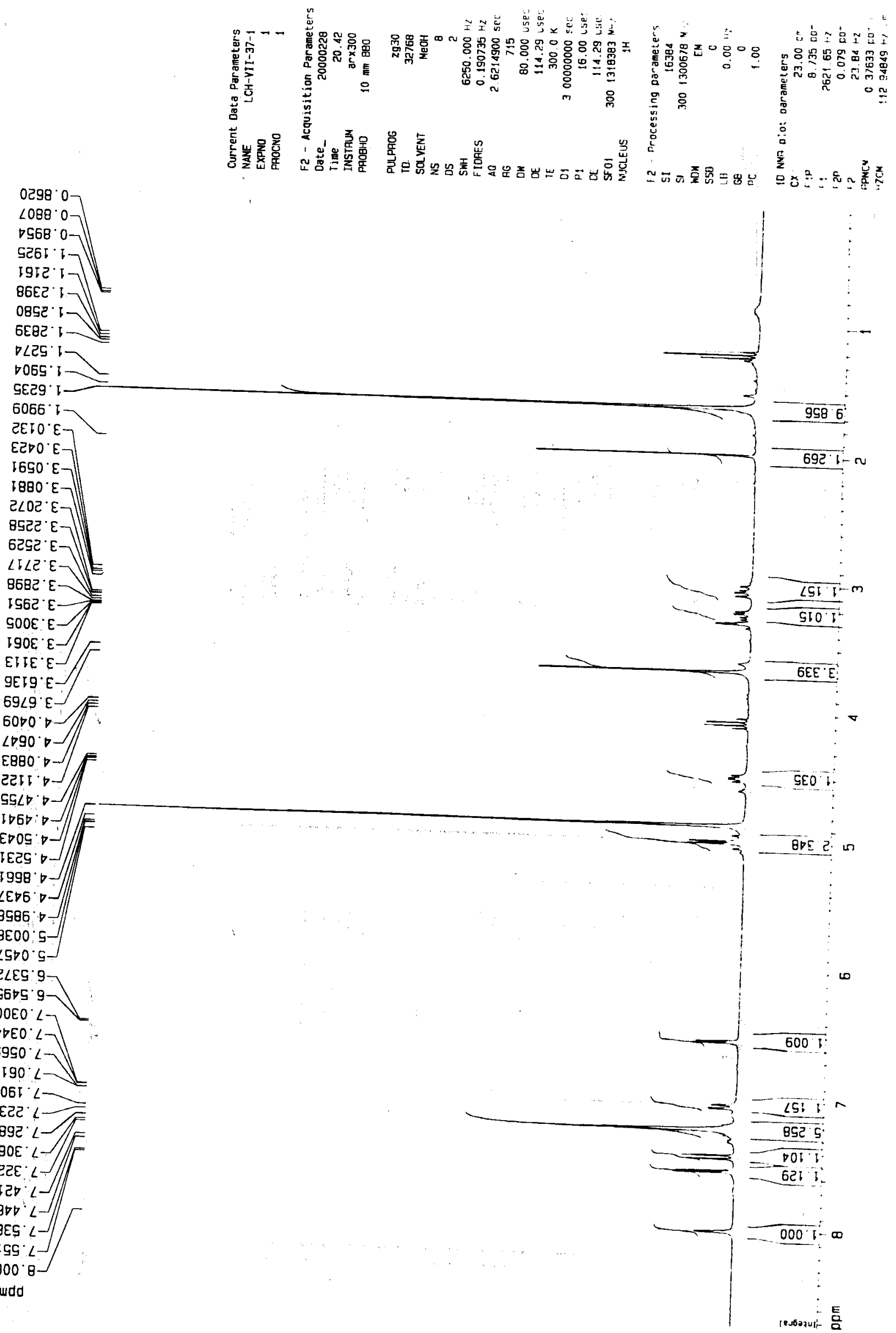


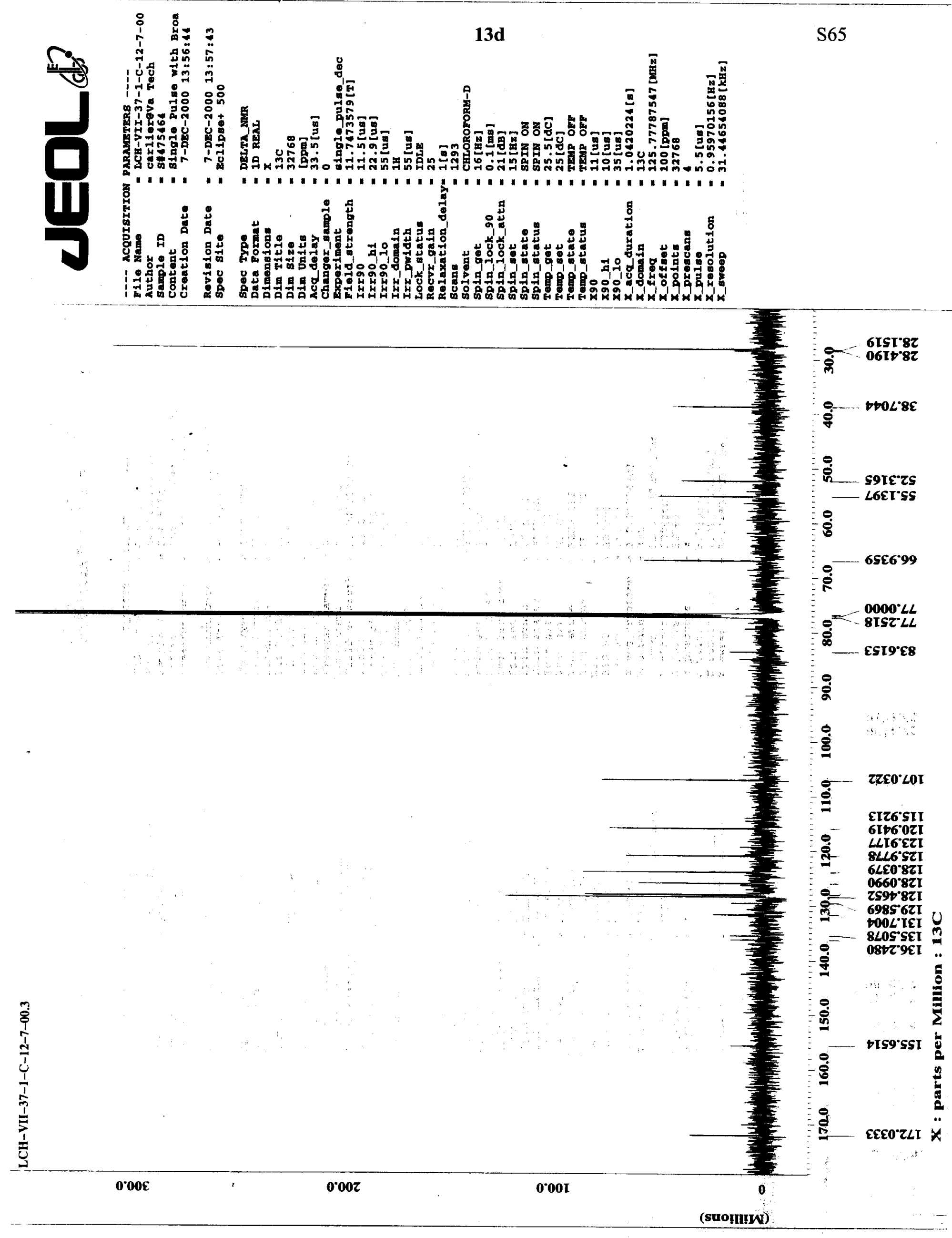



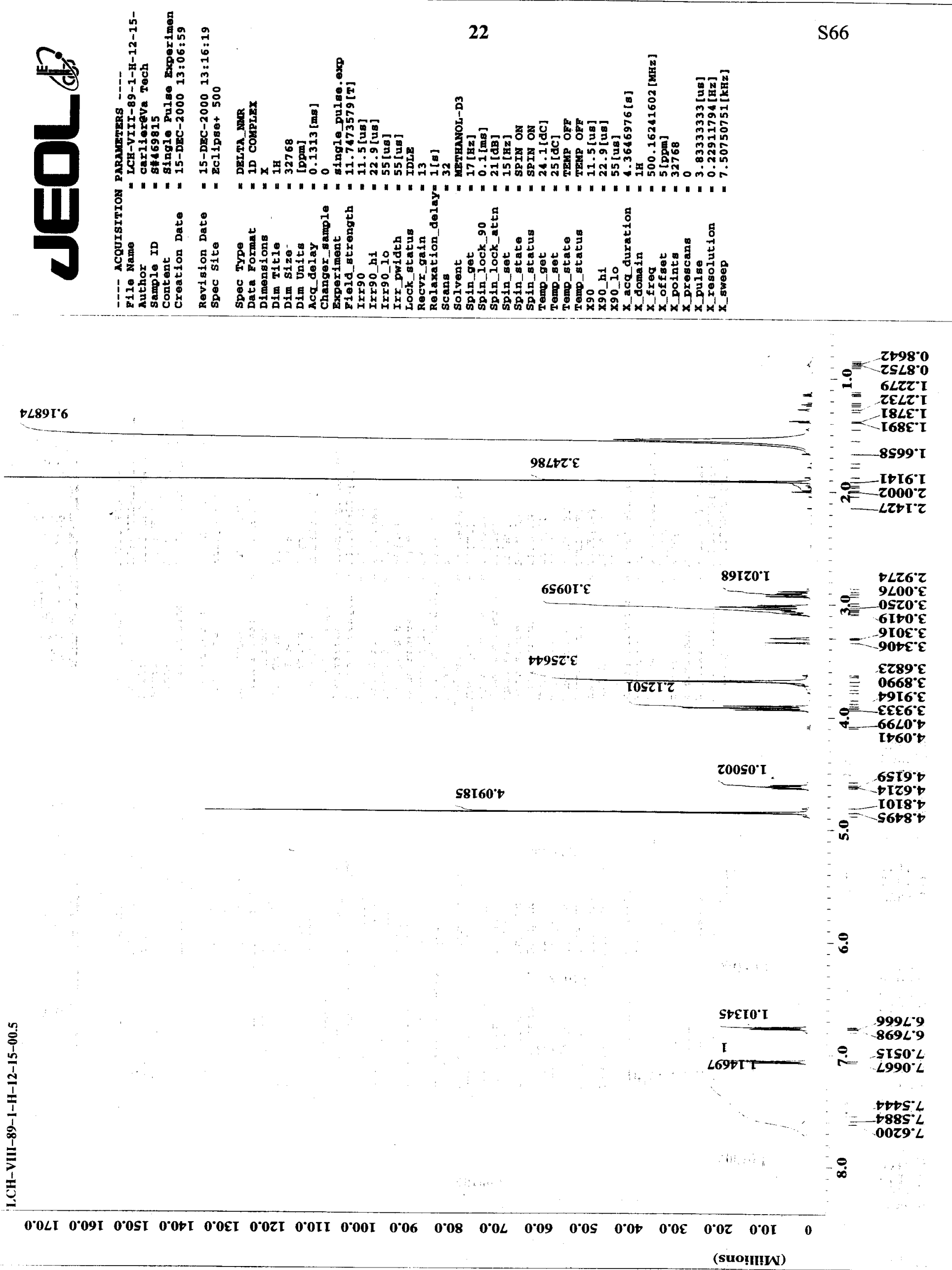

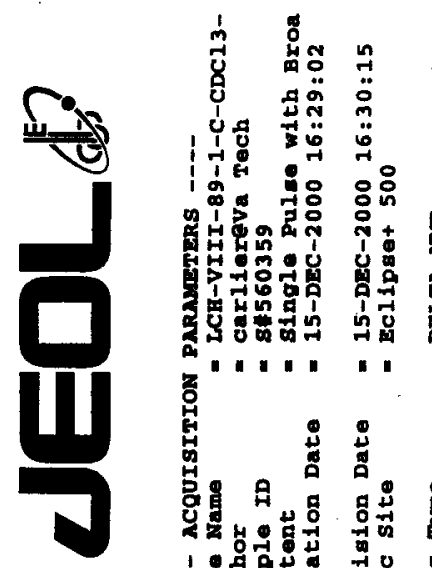

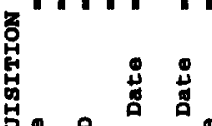
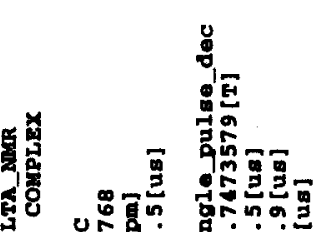

可

$=\frac{2}{3}$

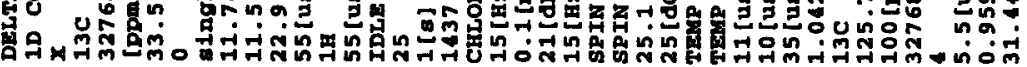

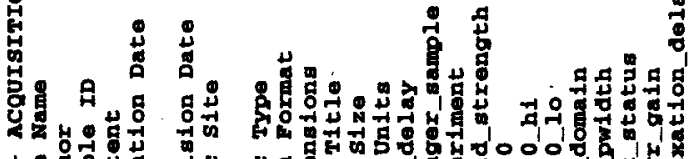

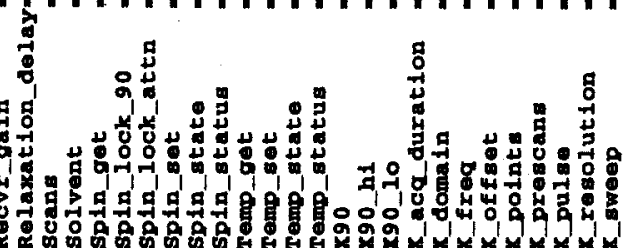

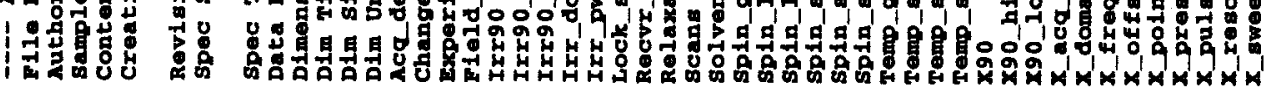

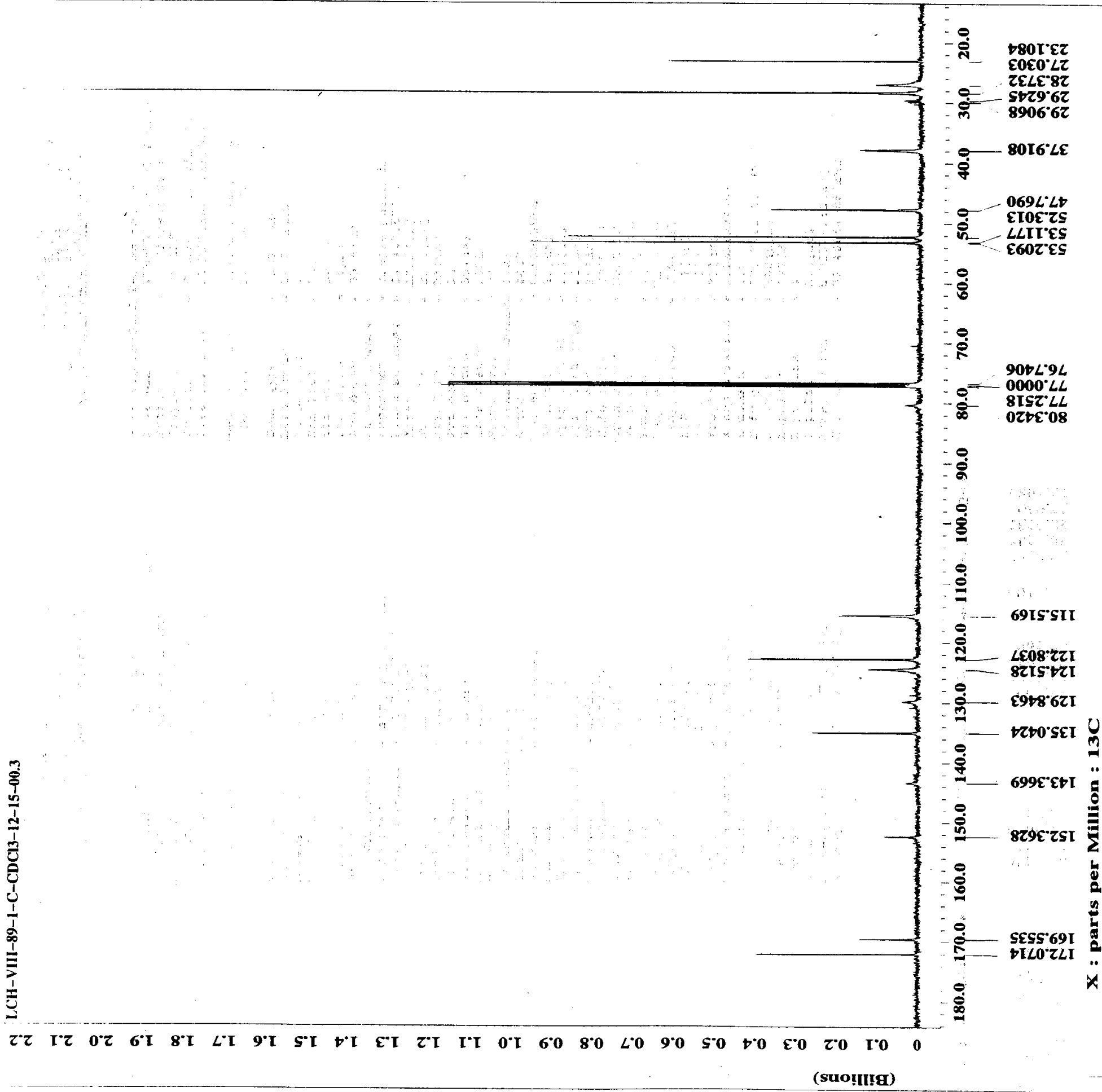

